\title{
Derivations with Values in the Ideal of $\tau$-Compact Operators Affiliated with a Semifinite von Neumann Algebra
}

\author{
A. Ber ${ }^{1}$, J. Huang ${ }^{2}$ (D) G. Levitina ${ }^{3}$, F. Sukochev ${ }^{2}$ \\ 1 Department of Mathematics, National University of Uzbekistan, Vuzgorodok, Tashkent, Uzbekistan 100174. \\ E-mail: ber@ucd.uz \\ 2 School of Mathematics and Statistics, University of New South Wales, Kensington, Sydney, NSW 2052, \\ Australia. E-mail: jinghao.huang@unsw.edu.au; f.sukochev@unsw.edu.au \\ 3 Mathematical Sciences Institute, Australian National University, Canberra, ACT 2600, Australia. \\ E-mail: Galina.Levitina@anu.edu.au
}

Received: 3 September 2020 / Accepted: 3 January 2022

Published online: 25 January 2022 - (C) The Author(s) 2022

\begin{abstract}
Let $\mathcal{M}$ be a semifinite von Neumann algebra with a faithful normal semifinite trace $\tau$ and let $\mathcal{A}$ be an arbitrary von Neumann subalgebra of $\mathcal{M}$. We characterize the class of symmetric ideals $\mathcal{E}$ in $\mathcal{M}$ such that derivations $\delta: \mathcal{A} \rightarrow \mathcal{E}$ are necessarily inner, which is a unification and far-reaching extension of the results due to Johnson and Parrott (J Funct Anal 11:39-61, 1972), due to Kaftal and Weiss (J Funct Anal 62:202-220, 1985), and due to Popa (J Funct Anal 71:393-408, 1987). In particular, we show that every derivation from $\mathcal{A}$ into the ideal $\mathcal{C}_{0}(\mathcal{M}, \tau)$ of all $\tau$-compact operators is inner, establishing a semifinite version of the Johnson-Parrott-Popa Theorem which is different from Popa and Rădulescu (Duke Math J 57(2):485-518, 1988, Theorem 1.1) and contrasts to the example of a non-inner derivation established in Popa and Rădulescu (1988, Theorem 1.2).
\end{abstract}

\section{Introduction}

Let $\mathcal{A}$ be a von Neumann algebra and let $J$ be an $\mathcal{A}$-bimodule. A derivation $\delta: \mathcal{A} \rightarrow J$ is a linear mapping satisfying $\delta(X Y)=\delta(X) Y+X \delta(Y), X, Y \in \mathcal{A}$. In particular, if $K \in J$, then $\delta_{K}(X):=X K-K X$ is a derivation. Such derivations implemented by elements in $J$ are called inner. One of the classical problems in operator algebra theory is the question whether every derivation from $\mathcal{A}$ into $J$ is automatically inner. During the past decades, a number of important special cases have been resolved (see e.g. $[4,36,37,48,54,57])$.

Kadison [38] and Sakai [58] gave an affirmative answer to the special case when the $\mathcal{A}$-bimodule $J$ coincides with the algebra $\mathcal{A}$ itself. Further, it was proved that every derivation from a von Neumann algebra into its arbitrary ideal is automatically inner $[12,13]$. However, when one considers more general $\mathcal{A}$-bimodules $J$ (see e.g. $[1,2,4-$ $11,35,44,55])$, there are examples of non-inner derivations for some specific $\mathcal{A}$ and $J$ (see e.g. [55], see also [7,10,59]). In the present paper, we concentrate on derivations with values in an ideal of $\mathcal{M}$, where $\mathcal{M}$ is an algebra such that $\mathcal{A} \subset \mathcal{M}$. 
Question 1.1. Assume that $\mathcal{M}$ is a semifinite von Neumann algebra equipped with a faithful normal semifinite trace $\tau$. Let $\mathcal{E}$ be an ideal in $\mathcal{M}$ and let $\mathcal{A}$ be an arbitrary von Neumann subalgebra of $\mathcal{M}$. Is every derivation $\delta$ from $\mathcal{A}$ into $\mathcal{E}$ necessarily inner?

It is desirable to identify the ideals $\mathcal{E}$ in $\mathcal{M}$ such that every derivation $\delta: \mathcal{A} \rightarrow \mathcal{E}$ is necessarily inner (see e.g. [33] and [36, Section 10.11]) and a number of mathematicians have been working on this problem since the 1960s (see e.g. $[10,12,13,16,32,33,37$, $38,44,54,58]$ ). Even though the special case when $\mathcal{E}=\mathcal{M}$ is still open (e.g. when $\mathcal{M}=B(\mathcal{H})$, the algebra of all bounded linear operators on a Hilbert space $\mathcal{H})$, there are results giving affirmative answers to this question under some additional conditions on the subalgebra $\mathcal{A}[53,61]$. For those who are interested in this special case, we refer to $[15,17,18,21,53,61]$.

Johnson and Parrott [37] considered the special case when the range of $\delta$ is contained in $K(\mathcal{H})$, the ideal of all compact operators on $\mathcal{H}$, and $\delta$ acts on a von Neumman subalgebra $\mathcal{A}$ of $B(\mathcal{H})$ having no certain type $I I_{1}$ factors as direct summands. The remaining case, when $\mathcal{A}$ is a type $I I_{1}$ von Neumann algebra was later resolved by Popa in [54].

Over the past decades, many authors have tried to establish a suitable semifinite version of the Johnson-Parrott-Popa Theorem [37,54]. In [44], the derivation problem initiated by Johnson and Parrott was studied in a more general setting where $B(\mathcal{H})$ is replaced with a semifinite von Neumann algebra $\mathcal{M}$ and $K(\mathcal{H})$ is replaced with the uniform norm closed ideal $\mathcal{J}(\mathcal{M})$ generated by all finite projections in $\mathcal{M}$. It is shown in [44] that if $\mathcal{A}$ is an abelian (or properly infinite) von Neumann subalgebra of $\mathcal{M}$ containing the center $\mathcal{Z}(\mathcal{M})$ of $\mathcal{M}$, then any derivation of $\mathcal{A}$ into $\mathcal{J}(\mathcal{M})$ is inner. Later, Popa and Rădulescu [55, Theorem 1.1] extended this result to the case when $\mathcal{A}$ is a von Neumann subalgebra of $\mathcal{M}$ and the finite type $I$ summand of $\mathcal{A}$ is locally compatible with the center of $\mathcal{M}$ (in particular, they resolved the case when $\mathcal{A}$ is a type $I I_{1}$ von Neumann subalgebra of $\mathcal{M}$, which was left untreated in [44]). Surprisingly, they established the existence of non-inner derivations $\delta: \mathcal{A} \rightarrow \mathcal{J}(\mathcal{M})$ for some specific semifinite von Neumann algebra $\mathcal{M}$ and abelian von Neumann subalgebra $\mathcal{A}$ of $\mathcal{M}$. The notion of the so-called generalized compacts $C(\mathcal{M})$ associated with a von Neumann algebra $\mathcal{M}$ was introduced by Wright [66] in 1984. Christensen [16] showed that derivations $\delta: \mathcal{M} \rightarrow C(\mathcal{M})$ are inner if $\mathcal{M}$ is properly infinite or injective. However, the case when $\mathcal{M}$ is a type $I I_{1}$ von Neumann algebra was a long-standing open question and was resolved by Galatan and Popa [32] in 2017.

However, for a general semifinite von Neumann algebra $\mathcal{M}$, there is another notion of compactness, so-called $\tau$-compactness, which comes from a semifinite trace $\tau$ defined on the algebra $\mathcal{M}$. Hence, it is natural to consider a problem, similar to that considered in [55], with the ideal $\mathcal{J}(\mathcal{M})$ of all compact operators replaced by the ideal $\mathcal{C}_{0}(\mathcal{M}, \tau)$ of all $\tau$-compact operators affiliated with $\mathcal{M}$ (see Sect. 2 for precise definitions). The following theorem is first main result of our paper.

Theorem 1.2. Assume that $\mathcal{M}$ is a semifinite von Neumann algebra equipped with a faithful normal semifinite trace $\tau$. Every derivation from an arbitrary von Neumann subalgebra $\mathcal{A}$ of $\mathcal{M}$ into $\mathcal{C}_{0}(\mathcal{M}, \tau)$ is inner.

Although, the ideals $\mathcal{C}_{0}(\mathcal{M}, \tau)$ and $\mathcal{J}(\mathcal{M})$ are quite similar in many respects (see Sect. 2), the result of Theorem 1.2 is in strong contrast with [55, Theorem 1.1], which is somewhat unexpected. Namely, the additional assumption on the type $I$ summand of the von Neumann subalgebra $\mathcal{A}$ which plays an important role in [55] could be dispensed with in our current setting. We note that the assumption that $\mathcal{A}$ is von Neumann 
subalgebra of $\mathcal{M}$ is sharp, that is, this assumption can not be relaxed to the assumption that $\mathcal{A}$ is a $C^{*}$-subalgebra of $\mathcal{M}$ for derivations into $\mathcal{J}(\mathcal{M})$ or into $\mathcal{C}_{0}(\mathcal{M}, \tau)$ (see [59, Example 4.1.8] and [10, Theorem 3.8]).

Derivations with values in ideals of a von Neumann algebra have important applications in the study of hyperreflexivity of operator spaces [50], commutants mod normed ideals [65], automorphisms and epimorphisms of operator algebras [12,13,39,40], etc. In Sect. 8, as an application of Theorem 1.2, we show that Question 1.1 has a positive answer for a very extensive class of symmetric ideals $\mathcal{E}$ of $\mathcal{M}$ by using techniques concerning noncommutative integration, which are completely different from those used in $[10,33,37,44,54,55]$.

Theorem 1.3. Assume that $\mathcal{M}$ is a semifinite von Neumann algebra equipped with a faithful normal semifinite trace $\tau$. If $E(\mathcal{M}, \tau)$ is a strongly symmetric space of $\tau$ measurable operators with the Fatou property, then every derivation $\delta$ from an arbitrary von Neumann subalgebra $\mathcal{A}$ of $\mathcal{M}$ into $E(\mathcal{M}, \tau) \cap \mathcal{C}_{0}(\mathcal{M}, \tau)$ is necessarily inner.

In [44], Kaftal and Weiss showed that derivations from $\mathcal{A}$ into $\mathcal{C}_{p}(\mathcal{M}, \tau):=L_{p}(\mathcal{M}, \tau) \cap$ $\mathcal{M}, p \geq 1$, are necessarily inner if $\mathcal{A}$ is an abelian (or properly infinite) von Neumann subalgebra of $\mathcal{M}$, where $L_{p}(\mathcal{M}, \tau)$ stands for the noncommutative $L_{p}$-space. However, the case of type $I$ (or type $I I_{1}$ ) von Neumann subalgebras was left unresolved in [44]. Theorem 1.3 generalizes the results due to Kaftal and Weiss [44] in two directions. Firstly, any additional conditions imposed on the von Neumann subalgebra $\mathcal{A}$ of $\mathcal{M}$ in [44] are removed. In particular, we completely resolve the cases unanswered in [44, Section 6]. Secondly, we have extended significantly the class of symmetric ideals associated with $\mathcal{M}$ for which the result is applicable. One should note that the Fatou property is an analogue of the so-called "dual normal" property (see e.g. [61]) and it is well-known that every derivation from a hyperfinite von Neumann algebra $\mathcal{A}$ into a dual normal $\mathcal{A}$-bimodule is inner (see e.g. [61, Theorem 2.4.3]). However, Theorem 1.3 is applicable for any von Neumann subalgebra $\mathcal{A}$ of $\mathcal{M}$. Moreover, this result complements Theorem 3.5 in our recent paper [10], where the derivations act on a larger class of algebras (i.e. $C^{*}$-subalgebras of $\mathcal{M}$ ) but the result is only applicable for symmetric operator spaces $E(\mathcal{M}, \tau)$ corresponding to Kantorovich-Banach fully symmetric function spaces $E(0, \infty)[24]$, that is, the additional assumption in [10] that the norm of $E(0, \infty)$ is order continuous is omitted in Theorem 1.3.

\section{Preliminaries}

In this section, we recall some notions of the theory of noncommutative integration.

In what follows, $\mathcal{H}$ is a Hilbert space and $B(\mathcal{H})$ is the $*$-algebra of all bounded linear operators on $\mathcal{H}$ equipped with the uniform norm $\|\cdot\|_{\infty}$, and $\mathbf{1}$ is the identity operator on $\mathcal{H}$. Let $\mathcal{M}$ be a von Neumann algebra on $\mathcal{H}$. We denote by $\mathcal{P}(\mathcal{M})$ the collection of all projections in $\mathcal{M}$, by $\mathcal{M}^{\prime}$ the commutant of $\mathcal{M}$ and by $\mathcal{Z}(\mathcal{M})$ the center of $\mathcal{M}$. For details on von Neumann algebra theory, the reader is referred to e.g. [22,41,42] or [62]. General facts concerning measurable operators may be found in $[49,60]$ (see also [63, Chapter IX] and the forthcoming book [30]). For convenience of the reader, some of the basic definitions are recalled.

2.1. $\tau$-measurable operators and generalized singular value functions. A closed, densely defined operator $X: \mathfrak{D}(X) \rightarrow \mathcal{H}$ with the domain $\mathfrak{D}(X)$ is said to be affiliated with 
$\mathcal{M}$ if $Y X \subseteq X Y$ for all $Y \in \mathcal{M}^{\prime}$, where $\mathcal{M}^{\prime}$ is the commutant of $\mathcal{M}$. A closed, densely defined operator $X: \mathfrak{D}(X) \rightarrow \mathcal{H}$ affiliated with $\mathcal{M}$ is said to be measurable if there exists a sequence $\left\{P_{n}\right\}_{n=1}^{\infty} \subset \mathcal{P}(\mathcal{M})$, such that $P_{n} \uparrow \mathbf{1}, P_{n}(\mathcal{H}) \subseteq \mathfrak{D}(X)$ and $\mathbf{1}-P_{n}$ is a finite projection (with respect to $\mathcal{M}$ ) for all $n$. The collection of all measurable operators with respect to $\mathcal{M}$ is denoted by $S(\mathcal{M})$, which is a unital $*$-algebra with respect to strong sums and products (denoted simply by $X+Y$ and $X Y$ for all $X, Y \in S(\mathcal{M})$ ).

Let $X$ be a self-adjoint operator affiliated with $\mathcal{M}$. We denote its spectral measure by $\left\{E^{X}\right\}$. It is well known that if $X$ is an operator affiliated with $\mathcal{M}$ with the polar decomposition $X=U|X|$, then $U \in \mathcal{M}$ and $E \in \mathcal{M}$ for all projections $E \in\left\{E^{|X|}\right\}$. Moreover, $X \in S(\mathcal{M})$ if and only if $E^{|X|}(\lambda, \infty)$ is a finite projection for some $\lambda>0$. It follows immediately that in the case when $\mathcal{M}$ is a von Neumann algebra of type $I$ I I or a type $I$ factor, we have $S(\mathcal{M})=\mathcal{M}$. For type $I I$ von Neumann algebras, this is no longer true. From now on, let $\mathcal{M}$ be a semifinite von Neumann algebra equipped with a faithful normal semifinite trace $\tau$.

An operator $X \in S(\mathcal{M})$ is called $\tau$-measurable if there exists a sequence $\left\{P_{n}\right\}_{n=1}^{\infty}$ in $P(\mathcal{M})$ such that $P_{n} \uparrow \mathbf{1}, P_{n}(\mathcal{H}) \subseteq \mathfrak{D}(X)$ and $\tau\left(\mathbf{1}-P_{n}\right)<\infty$ for all $n$. The collection $S(\mathcal{M}, \tau)$ of all $\tau$-measurable operators is a unital $*$-subalgebra of $S(\mathcal{M})$. It is well known that a linear operator $X$ belongs to $S(\mathcal{M}, \tau)$ if and only if $X \in S(\mathcal{M})$ and there exists $\lambda>0$ such that $\tau\left(E^{|X|}(\lambda, \infty)\right)<\infty$. Alternatively, an unbounded operator $X$ affiliated with $\mathcal{M}$ is $\tau$-measurable (see [31]) if and only if

$$
\tau\left(E^{|X|}(n, \infty)\right) \rightarrow 0, \quad n \rightarrow \infty
$$

Definition 2.1. Let a semifinite von Neumann algebra $\mathcal{M}$ be equipped with a faithful normal semi-finite trace $\tau$ and let $X \in S(\mathcal{M}, \tau)$. The generalized singular value function $\mu(X): t \rightarrow \mu(t ; X)$ of the operator $X$ is defined by setting

$$
\mu(s ; X)=\inf \{\|X P\|: P \in \mathcal{P}(\mathcal{M}) \text { with } \tau(\mathbf{1}-P) \leq s\} .
$$

An equivalent definition in terms of the distribution function of the operator $X$ is the following. For every self-adjoint operator $X \in S(\mathcal{M}, \tau)$, setting

$$
d_{X}(t)=\tau\left(E^{X}(t, \infty)\right), \quad t>0,
$$

we have (see e.g. [31])

$$
\mu(t ; X)=\inf \left\{s \geq 0: d_{|X|}(s) \leq t\right\} .
$$

It is well-known $[28,47]$ that if $A \in S(\mathcal{M}, \tau)$ and $B, C \in \mathcal{M}$, then

$$
\mu(t ; B A C) \leq\|B\|_{\infty}\|C\|_{\infty} \mu(t ; A), \mu\left(t ; A^{*}\right)=\mu(t ; A) .
$$

Consider the algebra $\mathcal{M}=L^{\infty}(0, \infty)$ of all Lebesgue measurable essentially bounded functions on $(0, \infty)$. The algebra $\mathcal{M}$ can be seen as an abelian von Neumann algebra acting via multiplication on the Hilbert space $\mathcal{H}=L^{2}(0, \infty)$, with the trace given by integration with respect to Lebesgue measure $m$. It is easy to see that the algebra of all $\tau$ measurable operators affiliated with $\mathcal{M}$ can be identified with the subalgebra $S(0, \infty)$ of the algebra of Lebesgue measurable functions $L_{0}(0, \infty)$ which consists of all functions $x$ such that $m(\{|x|>s\})$ is finite for some $s>0$. It should also be pointed out that the generalized singular value function $\mu(x)$ is precisely the decreasing rearrangement $\mu(x)$ of the function $|x|$ (see e.g. [46]) defined by

$$
\mu(t ; x)=\inf \{s \geq 0: m(\{|x| \geq s\}) \leq t\} .
$$


If $\mathcal{M}=B(\mathcal{H})$ (respectively, $l_{\infty}$ ) and $\tau$ is the standard trace $\operatorname{Tr}$ (respectively, the counting measure on $\mathbb{N})$, then it is not difficult to see that $S(\mathcal{M})=S(\mathcal{M}, \tau)=\mathcal{M}$. In this case, for $X \in S(\mathcal{M}, \tau)$ we have

$$
\mu(n ; X)=\mu(t ; X), \quad t \in[n, n+1), \quad n \geq 0 .
$$

The sequence $\{\mu(n ; X)\}_{n \geq 0}$ is just the sequence of singular values of the operator $X$.

If $X, Y \in S(\mathcal{M}, \tau)$, then $X$ is said to be submajorized by $Y$, denoted by $X \prec \prec Y$, if

$$
\int_{0}^{t} \mu(s ; X) d s \leq \int_{0}^{t} \mu(s ; Y) d s
$$

for all $t \geq 0$. In particular, for $x, y \in S(0, \infty), x \prec \prec y$ if and only if $\int_{0}^{t} \mu(s ; x) d s \leq$ $\int_{0}^{t} \mu(s ; y) d s, t \geq 0$.

The following proposition is an easy consequence of [29, Lemma 6.1] and [47, Lemma 3.3.5].

Proposition 2.2. Assume that $P_{1}, P_{2}, \cdots, P_{n} \in \mathcal{M}$ are projections with $P_{i} P_{j}=0$, $i \neq j$. Let $\alpha_{i}>0, i \in \mathbb{N}$, be such that $\sum_{i=1}^{n} \alpha_{i} \leq 1$. For every $X \in S(\mathcal{M}, \tau)$, we have

$$
\int_{0}^{a} \mu(t ; X) d t \geq \sum_{i=1}^{n} \int_{0}^{\alpha_{i} a} \mu\left(t ;\left|P_{i} X P_{i}\right|\right) d t=\sum_{i=1}^{n} \int_{0}^{\alpha_{i} a} \mu\left(t ; P_{i} X P_{i}\right) d t, \forall a>0 .
$$

Proof. Since $P_{i}$ are pairwise disjoint, it follows that

$$
\begin{aligned}
\mu\left(P_{1} X P_{1}+P_{2} X P_{2}+\cdots+P_{n} X P_{n}\right) & =\mu\left(\left|P_{1} X P_{1}+P_{2} X P_{2}+\cdots+P_{n} X P_{n}\right|\right) \\
& =\mu\left(\left|P_{1} X P_{1}\right|+\left|P_{2} X P_{2}\right|+\cdots+\left|P_{n} X P_{n}\right|\right) .
\end{aligned}
$$

Therefore, by [29, Lemma 6.1], we obtain that

$$
\int_{0}^{a} \mu\left(t ;\left|P_{1} X P_{1}\right|+\left|P_{2} X P_{2}\right|+\cdots+\left|P_{n} X P_{n}\right|\right) d t \leq \int_{0}^{a} \mu(t ; X) d t .
$$

The validity of (2.3) follows from [47, Lemma 3.3.5].

The following lemmas provide useful tools in proving the main theorem of the present paper.

Lemma 2.3. If $\left\{T_{i}\right\} \subset \mathcal{M}$ is a uniformly bounded net of self-adjoint operators converging to $T \in \mathcal{M}$ in the strong operator topology, then

$$
\liminf _{i} \tau\left(E^{T_{i}}(\varepsilon, \infty)\right) \geq \tau\left(E^{T}(\varepsilon, \infty)\right)
$$

for any $\varepsilon \in \mathbb{R}$.

Proof. Consider the characteristic function $\chi_{(\varepsilon, \infty)}$. There exists a sequence of positive continuous functions $f_{k}$ with compact support such that $f_{k} \uparrow \chi_{(\varepsilon, \infty)}$ pointwise. By [62, Lemma II 4.3], we have $f_{k}\left(T_{i}\right) \rightarrow_{\text {so }} f_{k}(T)$ for all $k \in \mathbb{N}$. Since $\tau$ is lower semicontinuous in the weak operator topology on a uniformly bounded set (see e.g. [62, Lemma II 2.5] and [63, Theorem VII 1.11]), it follows that

$$
\tau\left(f_{k}(T)\right) \leq \liminf _{i} \tau\left(f_{k}\left(T_{i}\right)\right) \leq \liminf _{i} \tau\left(\chi_{(\varepsilon, \infty)}\left(T_{i}\right)\right)=\liminf _{i} \tau\left(E^{T_{i}}(\varepsilon, \infty)\right) .
$$


Note that $f_{k} \uparrow \chi_{(\varepsilon, \infty)}$ implies $\sup _{k} f_{k}(T)=\chi_{(\varepsilon, \infty)}(T)=E^{T}(\varepsilon, \infty)$. Hence, using the normality of the trace $\tau$, we conclude that

$$
\tau\left(E^{T}(\varepsilon, \infty)\right)=\sup _{k \in \mathbb{N}} \tau\left(f_{k}(T)\right) \leq \liminf _{i} \tau\left(E^{T_{i}}(\varepsilon, \infty)\right) .
$$

Lemma 2.4. For every $X \in \mathcal{M}$ and $t>0, \tau\left(E^{|X|}(a, \infty)\right)>t$ if and only if $\mu(s ; X)>a$ for all $s \in[0, t]$.

Proof. Necessity. By (2.1), we have $\mu(t ; X)=\inf \left\{s \geq 0: \tau\left(E^{|X|}(s, \infty)\right) \leq t\right\}$. Assume by contradiction that $\mu(t ; X) \leq a$, then $\inf \left\{s \geq 0: \tau\left(E^{|X|}(s, \infty)\right) \leq t\right\} \leq a$ and therefore $\tau\left(E^{|X|}(a+\varepsilon, \infty)\right) \leq t$ for any $\varepsilon>0$. Since the distribution function $d_{|X|}(\cdot)$ is right-continuous (see e.g. [31]), it follows that $\tau\left(E^{|X|}(a, \infty)\right) \leq t$, which is a contradiction.

Sufficiency. By assumption, we have that $\mu(t ; X)>a$. Using again (2.1), we obtain that $\inf \left\{s \geq 0: \tau\left(E^{|X|}(s, \infty)\right) \leq t\right\}>a$, and therefore $\tau\left(E^{|X|}(a, \infty)\right)>t$.

\subsection{Symmetric spaces.}

Definition 2.5. A linear subspace $E$ of $S(\mathcal{M}, \tau)$ equipped with a complete norm $\|\cdot\|_{E}$, is called symmetric space (of $\tau$-measurable operators) if $X \in S(\mathcal{M}, \tau), Y \in E$ and $\mu(X) \leq \mu(Y)$ imply that $X \in E$ and $\|X\|_{E} \leq\|Y\|_{E}$.

It is well-known that any symmetric space $E$ is a normed $\mathcal{M}$-bimodule, that is $A X B \in$ $E$ for any $X \in E, A, B \in \mathcal{M}$ and $\|A X B\|_{E} \leq\|A\|_{\infty}\|B\|_{\infty}\|X\|_{E}[28,30]$.

A symmetric space $E(\mathcal{M}, \tau) \subset S(\mathcal{M}, \tau)$ is called strongly symmetric if its norm $\|\cdot\|_{E}$ has the additional property that $\|X\|_{E} \leq\|Y\|_{E}$ whenever $X, Y \in E(\mathcal{M}, \tau)$ satisfy $X \prec \prec Y$. In addition, if $X \in S(\mathcal{M}, \tau), Y \in E(\mathcal{M}, \tau)$ and $X \prec \prec Y$ imply that $X \in E(\mathcal{M}, \tau)$ and $\|X\|_{E} \leq\|Y\|_{E}$, then $E(\mathcal{M}, \tau)$ is called fully symmetric space (of $\tau$-measurable operators).

A symmetric space $E(\mathcal{M}, \tau)$ is said to have the Fatou property if for every upwards directed net $\left\{X_{\beta}\right\}$ in $E(\mathcal{M}, \tau)^{+}$, satisfying $\sup _{\beta}\left\|X_{\beta}\right\|_{E}<\infty$, there exists an element $X \in E(\mathcal{M}, \tau)^{+}$such that $X_{\beta} \uparrow X$ in $E(\mathcal{M}, \tau)$ and $\|X\|_{E}=\sup _{\beta}\left\|X_{\beta}\right\|_{E}$. Examples such as Schatten-von Neumann operator ideals, Lorentz operator ideals, Orlicz operator ideals, etc. all have symmetric norms which have the Fatou property.

If $E(\mathcal{M}, \tau)$ is a symmetric space, then the carrier projection $c_{E} \in \mathcal{P}(\mathcal{M})$ is defined by setting

$$
c_{E}=\bigvee\{P: P \in P(\mathcal{M}), P \in E(\mathcal{M}, \tau)\}
$$

If $E(\mathcal{M}, \tau)$ is a symmetric space, then the Köthe dual $E(\mathcal{M}, \tau)^{\times}$of $E(\mathcal{M}, \tau)$ is defined by

$$
E(\mathcal{M}, \tau)^{\times}=\left\{X \in S(\mathcal{M}, \tau): \sup _{\|Y\|_{E} \leq 1, Y \in E} \tau(|X Y|)<\infty\right\},
$$

and for every $X \in E(\mathcal{M}, \tau)^{\times}$, we set $\|X\|_{E^{\times}}=\sup \left\{\tau(|Y X|): Y \in E(\mathcal{M}, \tau),\|Y\|_{E} \leq\right.$ 1) (see e.g. [28, Section 5.2], see also [25,47]). It is well-known that $\|\cdot\|_{E^{\times}}$is a norm on $E(\mathcal{M}, \tau)^{\times}$if and only if the carrier projection $c_{E}$ of $E(\mathcal{M}, \tau)$ is equal to 1 . In this case, for a strongly symmetric space $E(\mathcal{M}, \tau)$, the following statements are equivalent $[26,28]$. 
- $E(\mathcal{M}, \tau)$ has the Fatou property.

- $E(\mathcal{M}, \tau)^{\times \times}=E(\mathcal{M}, \tau)$ and $\|X\|_{E}=\|X\|_{E^{\times \times}}$for all $X \in E(\mathcal{M}, \tau)$.

A wide class of symmetric operator spaces associated with the von Neumman algebra $\mathcal{M}$ can be constructed from concrete symmetric function spaces studied extensively in e.g. [46]. Let $\left(E(0, \infty),\|\cdot\|_{E(0, \infty)}\right)$ be a symmetric function space on the semi-axis $(0, \infty)$. The pair

$$
E(\mathcal{M}, \tau)=\{X \in S(\mathcal{M}, \tau): \mu(X) \in E(0, \infty)\}, \quad\|X\|_{E(\mathcal{M}, \tau)}:=\|\mu(X)\|_{E(0, \infty)}
$$

is a symmetric operator space affiliated with $\mathcal{M}$ with $c_{E}=\mathbf{1}$ [45] (see also [47]). Further, we have

$$
L_{1} \cap L_{\infty}(\mathcal{M}, \tau) \subset E(\mathcal{M}, \tau) \subset\left(L_{1}+L_{\infty}\right)(\mathcal{M}, \tau)
$$

For convenience, we denote $\|\cdot\|_{E(\mathcal{M}, \tau)}$ by $\|\cdot\|_{E}$. Many properties of symmetric spaces, such as reflexivity, Fatou property, order continuity of the norm as well as Köthe duality carry over from commutative symmetric function space $E(0, \infty)$ to its noncommutative counterpart $E(\mathcal{M}, \tau)$ (see e.g. [28, Theorem 53 and Theorem 54] and [26]).

2.3. The ideal of $\tau$-compact operators. A projection $P \in \mathcal{P}(\mathcal{M})$ is called $\tau$-finite if $\tau(P)<\infty$. If $P \in \mathcal{P}(\mathcal{M})$ is $\tau$-finite, then $P$ is a finite projection. For $X \in \mathcal{M}$, we denote by $l(X)$ and $r(X)$ the left support and right support of $X$, respectively. In particular, for a self-adjoint operator $X \in \mathcal{M}$, we denote by $s(X)$ its support. Every projection $P \in \mathcal{M}$ has a central support which is defined as the smallest projection in the center $\mathcal{Z}(\mathcal{M})$ containing $P$ as a subprojection. The two-sided ideal $\mathcal{F}(\mathcal{M}, \tau)$ in $\mathcal{M}$ consisting of all elements of $\tau$-finite range is defined by setting

$$
\mathcal{F}(\mathcal{M}, \tau)=\{X \in \mathcal{M}: \tau(r(X))<\infty\}=\{X \in \mathcal{M}: \tau(l(X))<\infty\} .
$$

The ideal $\mathcal{C}_{0}(\mathcal{M}, \tau)$ of all $\tau$-compact bounded operators can be described as the closure in the norm $\|\cdot\|_{\infty}$ of the linear span of all $\tau$-finite projections [47, Definition 2.6.8]. Equivalently, $\mathcal{C}_{0}(\mathcal{M}, \tau)$ is set of all elements $X \in \mathcal{M}$ such that $\tau\left(E^{|X|}(\lambda, \infty)\right)<$ $\infty$ for every $\lambda>0$ (see e.g. [30, Chapter II, Section 4]). The space $\mathcal{C}_{0}(\mathcal{M}, \tau)$ is associated to the ideal of essentially bounded functions vanishing at infinity (see [47, Lemma 2.6.9]), that is,

$$
\mathcal{C}_{0}(\mathcal{M}, \tau)=\left\{A \in S(\mathcal{M}, \tau): \mu(A) \in L_{\infty}(0, \infty), \mu(\infty ; A):=\lim _{t \rightarrow \infty} \mu(t ; A)=0\right\}
$$

In particular, if $\tau$ is finite, then $\mathcal{M}=\mathcal{C}_{0}(\mathcal{M}, \tau)$ (see e.g. [47, Page 64]). The space $S_{0}(\mathcal{M}, \tau)$ of $\tau$-compact operators is the space associated to the algebra of functions from $S(0, \infty)$ vanishing at infinity, that is,

$$
S_{0}(\mathcal{M}, \tau)=\{A \in S(\mathcal{M}, \tau): \mu(\infty ; A)=0\}
$$

This is a two-sided ideal in $S(\mathcal{M}, \tau)$ and, clearly, $\mathcal{C}_{0}(\mathcal{M}, \tau)=S_{0}(\mathcal{M}, \tau) \cap \mathcal{M}$.

The following lemma provides a sufficient condition for an operator $X \in \mathcal{M}$ to be not $\tau$-compact. This condition plays a crucial role in the proof of Theorem 4.2. 
Lemma 2.6. Let $X \in \mathcal{M}$ and $\left\{\alpha_{i}>0\right\}_{i}$ be an arbitrary sequence of real numbers increasing to infinity. If there exists a number $c>0$ such that

$$
\int_{0}^{\alpha_{i}} \mu(t ; X) d t \geq \alpha_{i} c
$$

for every $\alpha_{i}$, then $\mu(t ; X) \geq c$ for all $t>0$, that is, $X$ is not $\tau$-compact. In other words, if $\mu(X) \succ \succ c$, then $\mu(X) \geq c$.

Proof. Assume by contradiction that $\mu\left(n_{0} ; X\right)<c$ for some $n_{0}>0$ and therefore $\mu(t ; X) \leq \mu\left(n_{0} ; X\right)<c$ for every $t \geq n_{0}$. Noticing that $\|X\|_{\infty}<\infty$, we have

$$
\begin{aligned}
n_{0} \cdot\|X\|_{\infty}+\left(\alpha_{i}-n_{0}\right) \mu\left(n_{0} ; X\right) & \geq \int_{0}^{n_{0}}\|X\|_{\infty} d t+\int_{n_{0}}^{\alpha_{i}} \mu\left(n_{0} ; X\right) d t \\
& \geq \int_{0}^{n_{0}} \mu(t ; X) d t+\int_{n_{0}}^{\alpha_{i}} \mu(t ; X) d t \\
& =\int_{0}^{\alpha_{i}} \mu(t ; X) d t \geq \alpha_{i} c
\end{aligned}
$$

for any $\alpha_{i} \geq n_{0}$. Thus, we obtain that $\mu\left(n_{0} ; X\right) \geq \frac{\alpha_{i} c-n_{0}\|X\|_{\infty}}{\alpha_{i}-n_{0}}$ for every $\alpha_{i} \geq n_{0}$. By assumption, we have that $\alpha_{i} \rightarrow_{i} \infty$ as $i \rightarrow \infty$, and therefore, it follows that $\mu\left(n_{0} ; X\right) \geq c$, which is a contradiction. Thus, $\mu(t ; X) \geq c$ for all $t>0$, which implies that the operator $X$ is not $\tau$-compact.

Recall that $\mathcal{J}(\mathcal{M})$ is the uniform norm closure of the linear span of all finite projections in $\mathcal{M}$. It is known that $\mathcal{C}_{0}(\mathcal{M}, \tau) \subset \mathcal{J}(\mathcal{M})$ for any semifinite algebra $\mathcal{M}$ and $\mathcal{C}_{0}(\mathcal{M}, \tau)=\mathcal{J}(\mathcal{M})$ whenever $\mathcal{M}$ is a factor (see e.g. [55, 2.1.1.]).

Remark 2.7. For a semifinite von Neumann algebra $\mathcal{M}$ equipped with a faithful normal semifinite trace $\tau$, it is easy to see that $\mathcal{C}_{0}(\mathcal{M}, \tau) \neq \mathcal{J}(\mathcal{M})$ if and only if there exists a finite projection $P \in \mathcal{M}$ such that $\tau(P)=\infty$ (see e.g. [43, Theorem 1.3]).

We end this section with the following theorem, which gives a necessary and sufficient condition on the algebra $\mathcal{M}$ for the existence of a faithful normal semifinite trace $\tau$ on $\mathcal{M}$ with $\mathcal{C}_{0}(\mathcal{M}, \tau) \varsubsetneqq \mathcal{J}(\mathcal{M})$.

Theorem 2.8. Let $\mathcal{M}$ be a semifinite von Neumann algebra. The following conditions are equivalent:

(i). There exists a faithful normal semifinite trace $\tau$ on $\mathcal{M}$ such that $\mathcal{C}_{0}(\mathcal{M}, \tau) \neq \mathcal{J}(\mathcal{M})$; (ii). $\operatorname{dim}(\mathcal{Z}(\mathcal{M}))=\infty$.

Proof. (i) $\Rightarrow$ (ii). Assume by contradiction that $\operatorname{dim}(\mathcal{Z}(\mathcal{M}))<\infty$. We denote by $E_{1}$, $\ldots, E_{n}, n \in \mathbb{N}$, the finite family of atoms in $\mathcal{Z}(\mathcal{M})$. It is clear that $\mathcal{M}_{E_{k}}$ is a semifinite factor for all $k=1, \ldots, n$. For every $k=1, \ldots, n$, fix a trace $\tau_{k}$ on $\mathcal{M}_{E_{k}}$. It is clear that $\tau(X)=\sum_{k=1}^{n} \alpha_{k} \tau_{k}\left(X E_{k}\right)$ for some $\alpha_{k}>0$.

By Remark 2.7, we can find a finite projection $P \in \mathcal{P}(\mathcal{M})$ such that $\tau(P)=\infty$. Therefore, $\tau_{k}\left(P E_{k}\right)=\infty$ for some $k$. However, this is impossible since $P E_{k}$ is a finite projection in the factor $\mathcal{M}_{E_{k}}$. This contradiction shows that $\operatorname{dim}(\mathcal{Z}(\mathcal{M}))=\infty$.

(ii) $\Rightarrow$ (i). Let $\tau^{\prime}$ be an arbitrary faithful normal semifinite trace on $\mathcal{M}$. By the assumption, there exists a sequence of pairwise disjoint non-zero projections $\left\{E_{n}\right\}_{n=1}^{\infty} \subset \mathcal{Z}(\mathcal{M})$ such that $\bigvee_{n=1}^{\infty} E_{n}=\mathbf{1}$. In every algebra $\mathcal{M}_{E_{n}}$, there exists a non-zero finite projection $P_{n}$. If for some $n$ we have that $\tau^{\prime}\left(P_{n}\right)=\infty$, then the assertion follows from Remark 2.7. 
Assume that $\tau^{\prime}\left(P_{n}\right)<\infty$ for all $n$. Since $P_{n} \in \mathcal{M}_{E_{n}}$ and $E_{n}$ are pairwise disjoint, it follows that the central supports of $P_{n}$ are pairwise disjoint. Hence, $P:=\bigvee_{n=1}^{\infty} P_{n}$ is also a finite projections. Set $\tau(X):=\sum_{n=1}^{\infty} n \tau^{\prime}\left(X E_{n}\right) / \tau^{\prime}\left(P_{n}\right)$. Clearly, $\tau$ is a faithful normal semifinite trace on $\mathcal{M}$ and $\tau(P)=\infty$. By Remark 2.7, we obtain the validity of (i).

\section{Preliminaries on Derivations}

In the present paper, we consider derivations $\delta$ from an arbitrary von Neumann subalgebra $\mathcal{A}$ of $\mathcal{M}$ into $\mathcal{C}_{0}(\mathcal{M}, \tau)$, that is, $\delta: \mathcal{A} \rightarrow \mathcal{C}_{0}(\mathcal{M}, \tau)$ is a linear mapping satisfying $\delta(X Y)=\delta(X) Y+X \delta(Y), X, Y \in \mathcal{A}$. A derivation is called skew-adjoint if $\delta=-\delta^{*}$, where $\delta^{*}$ is a derivation defined by $\delta^{*}(X)=\left(\delta\left(X^{*}\right)\right)^{*}, x \in \mathcal{A}$. Actually, we can assume that the derivation $\delta$ is skew-adjoint because every derivation $\delta: \mathcal{A} \rightarrow \mathcal{C}_{0}(\mathcal{M}, \tau)$ can be decomposed into skew-adjoint components $\delta=\delta_{1}+i \cdot \delta_{2}$, where

$$
\delta_{1}(X):=\frac{\delta(X)-\delta\left(X^{*}\right)^{*}}{2} \text { and } \delta_{2}(X):=\frac{\delta(X)+\delta\left(X^{*}\right)^{*}}{2 i}
$$

Remark 3.1. Assume that there exists an operator $T \in \mathcal{M}$ such that the skew-adjoint derivation $\delta=\delta_{T}=[\cdot, T]$. For every $X \in \mathcal{A}$, we have

$$
\left[X, T-T^{*}\right]=[X, T]-\left[X, T^{*}\right]=[X, T]+\left[X^{*}, T\right]^{*}=\delta(X)+\left(\delta\left(X^{*}\right)\right)^{*}=0,
$$

which implies that $\operatorname{Im}(T)=\frac{T-T^{*}}{2 i} \in \mathcal{A}^{\prime}$. Thus, for every $X \in \mathcal{A}$, we have

$$
\delta(X)=[X, T]=[X, \operatorname{Re}(T)+i \operatorname{Im}(T)]=[X, \operatorname{Re}(T)] .
$$

Hence, without loss of generality, we can always assume that the operator $T$ implementing a skew-adjoint derivation $\delta$ is self-adjoint.

In the following, we need to consider several types of reductions of a given derivation $\delta: \mathcal{A} \rightarrow \mathcal{C}_{0}(\mathcal{M}, \tau)$. The first one is reduction of $\delta$ by a given central projection in the algebra $\mathcal{M}$.

Lemma 3.2. Let $\delta: \mathcal{A} \rightarrow \mathcal{C}_{0}(\mathcal{M}, \tau)$ be a derivation and let $Z \in \mathcal{Z}(\mathcal{M})$ be a projection. The mapping $\delta^{(Z)}: \mathcal{A}_{Z} \rightarrow Z \mathcal{C}_{0}(\mathcal{M}, \tau) Z=\mathcal{C}_{0}\left(\mathcal{M}_{Z}, \tau\right)$ given by $\delta^{(Z)}(X Z)=Z \delta(X) Z$, $X \in \mathcal{A}$, is a well-defined derivation from the induced von Neumann algebra $\mathcal{A}_{Z}$ into $Z \mathcal{C}_{0}(\mathcal{M}, \tau) Z$.

Proof. If $A, B \in \mathcal{A}$ such that $A Z=B Z$, then

$$
\begin{aligned}
\delta^{(Z)} & (A Z)-\delta^{(Z)}(B Z) \\
& =Z \delta(A) Z-Z \delta(B) Z \\
& =Z \delta\left((A-B) E^{|A-B|}(0, \infty)\right) Z \\
& =Z \delta(A-B) \cdot E^{|A-B|}(0, \infty) Z+Z(A-B) \cdot \delta\left(E^{|A-B|}(0, \infty)\right) Z \\
& =Z \delta(A-B) \cdot E^{|A-B|}(0, \infty) Z .
\end{aligned}
$$

Since $Z \in \mathcal{Z}(\mathcal{M})$, it follows that $E^{|A-B|}(0, \infty) Z$ is a projection with $E^{|A-B|}(0, \infty) Z \leq$ $E^{|A-B|}(0, \infty)$. However, the assumption, $(A-B) E^{|A-B|}(0, \infty) Z=(A-B) Z=0$ 
implies that $E^{|A-B|}(0, \infty) Z=0$ and therefore $\delta^{(Z)}(A Z)=\delta^{(Z)}(B Z)$. Moreover, for every $X, Y \in \mathcal{A}$, we have

$$
\begin{aligned}
\delta^{(Z)}(Z X Z \cdot Z Y Z) & =\delta^{(Z)}(Z X Y Z)=Z \delta(X Y) Z \\
& =Z \delta(X) Y Z+Z X \delta(Y) Z \\
& =Z \delta(X) Z \cdot Z Y Z+Z X Z \cdot Z \delta(Y) Z \\
& =\delta^{(Z)}(Z X Z) Z Y Z+Z X Z \delta^{(Z)}(Z Y Z),
\end{aligned}
$$

which implies that $\delta^{(Z)}$ is a well-defined derivation.

The other reduction of $\delta: \mathcal{A} \rightarrow \mathcal{C}_{0}(\mathcal{M}, \tau)$ we intend to use depends on the type of the algebra $\mathcal{A}$ with an additional assumption that $\left.\delta\right|_{\mathcal{Z}(\mathcal{A})}$ vanishes. For every $Z \in \mathcal{Z}(\mathcal{A})$, the center of $\mathcal{A}$, the mapping $Z \delta(\cdot) Z$ is a derivation from $\mathcal{A}$ into $\mathcal{C}_{0}(\mathcal{M}, \tau)$. Moreover, if $\delta$ vanishes on $\mathcal{Z}(\mathcal{A})$, then $Z \delta(\cdot) Z$ is a derivation from $\mathcal{A}_{Z}$ into $Z \mathcal{C}_{0}(\mathcal{M}, \tau) Z$, which coincides with $\delta(\cdot)$ on $\mathcal{A}_{Z}$. Let $Z_{1}, Z_{2}$ be two projections in $\mathcal{M}$ such that $Z_{1} Z_{2}=0$. For elements $X_{1} \in \mathcal{M}_{Z_{1}}=Z_{1} \mathcal{M} Z_{1}$ and $X_{2} \in \mathcal{M}_{Z_{2}}=Z_{2} \mathcal{M} Z_{2}$, we frequently denote $X_{1}+X_{2}$ by $X_{1} \oplus X_{2}$.

Lemma 3.3. Let $\delta: \mathcal{A} \rightarrow \mathcal{C}_{0}(\mathcal{M}, \tau)$ be a derivation such that $\left.\delta\right|_{\mathcal{Z}(\mathcal{A})}=0$. If for $Z_{1}, Z_{2} \in \mathcal{Z}(\mathcal{A})$ with $Z_{1} Z_{2}=0,\left.\delta\right|_{\mathcal{A}_{Z_{1}}}$ and $\left.\delta\right|_{\mathcal{A}_{Z_{2}}}$ are inner derivations implemented by $T_{1} \in Z_{1} \mathcal{C}_{0}(\mathcal{M}, \tau) Z_{1}$ and $T_{2} \in Z_{2} \mathcal{C}_{0}(\mathcal{M}, \tau) Z_{2}$, then $\left.\delta\right|_{\mathcal{A}_{Z_{1}+Z_{2}}}$ is implemented by $T_{1} \oplus T_{2}$.

Proof. For every $X \in \mathcal{A}_{Z_{1}+Z_{2}}$, we have

$$
\begin{aligned}
\delta(X) & =\delta\left(X Z_{1}+X Z_{2}\right)=\delta\left(X Z_{1}\right)+\delta\left(X Z_{2}\right)=\delta_{T_{1}}\left(X Z_{1}\right)+\delta_{T_{2}}\left(X Z_{2}\right) \\
& =\left[X Z_{1}, T_{1}\right]+\left[X Z_{2}, T_{2}\right]=\left[X, T_{1}+T_{2}\right]=\delta_{T_{1}+T_{2}}(X),
\end{aligned}
$$

which completes the proof.

Lemma 3.3 allows us to make the following reduction of the problem considered in this paper.

Remark 3.4. Let $P_{1}, P_{2}, P_{3} \in \mathcal{Z}(\mathcal{A})$ be the central partition of unity (some of $P_{i}$ can be zero), such that $\mathcal{A}_{P_{1}}$ is of type $I_{\text {fin }}, \mathcal{A}_{P_{2}}$ is of type $\mathrm{II}_{1}, \mathcal{A}_{P_{3}}$ is properly infinite. Assume that $\delta: \mathcal{A} \rightarrow \mathcal{C}_{0}(\mathcal{M}, \tau)$ vanishes on $\mathcal{Z}(\mathcal{A})$. By reducing $\delta$ to the algebras $\mathcal{A}_{P_{i}}, i=1,2,3$, to prove that $\delta$ is inner derivation, it is sufficient to consider separately the cases when $\mathcal{A}$ is type $\mathrm{I}$, type $\mathrm{II}_{1}$ or properly infinite. As we show in Sect. 4 (see Remark 4.4), the assumption that $\delta$ vanishes on $\mathcal{Z}(\mathcal{A})$ can be imposed without loss of generality.

Next, we introduce a special subset $K_{\delta}$ of the algebra $\mathcal{M}$ generated by derivation $\delta: \mathcal{A} \rightarrow \mathcal{M}$. As we prove later, for any derivation $\delta: \mathcal{A} \rightarrow \mathcal{C}_{0}(\mathcal{M}, \tau), K_{\delta}$ contains the operator implementing $\delta$.

Definition 3.5. For a skew-adjoint derivation $\delta: \mathcal{A} \rightarrow \mathcal{M}$, we define by $K_{\delta}$ the weak* (or ultraweak) operator closure of $\operatorname{co}\left\{U \delta\left(U^{*}\right) \mid U \in \mathcal{U}(\mathcal{A})\right\}$, where $\operatorname{co}(S)$ denotes the convex hull of a set $S$.

Remark 3.6. Recall that the strong operator closure, the weak operator closure and the weak* operator closure of the convex hull of a uniformly bounded set in $\mathcal{M}$ coincide (see e.g. [62, Chapter II, Lemma 2.5] and [20, Chapter IX, Corollary 5.2]). By Ringrose's 
theorem [56] (see also [5, Theorem 3.1]), derivation $\delta: \mathcal{A} \rightarrow \mathcal{C}_{0}(\mathcal{M}, \tau)$ is bounded and therefore, the set $\{\delta(U) \mid U \in \mathcal{U}(\mathcal{A})\}$ is uniformly bounded. Thus,

$$
K_{\delta}=\overline{c o}^{w o}\left\{U \delta\left(U^{*}\right) \mid U \in \mathcal{U}(\mathcal{A})\right\}=\overline{c o}^{s o}\left\{U \delta\left(U^{*}\right) \mid U \in \mathcal{U}(\mathcal{A})\right\},
$$

where $\overline{c o}^{s o}(S)$ (respectively, $\overline{c o}^{\text {wo }}(S)$ ) denotes the strong operator closure (respectively, weak operator closure) of convex hull of a set $S$. In particular, $\|X\|_{\infty} \leq\|\delta\|_{\mathcal{A} \rightarrow \mathcal{M}}$ for every $X \in K_{\delta}$. Furthermore, since $\delta$ is assumed to be skew-adjoint, using Leibniz rule, for any unitary $U \in \mathcal{A}$, we have

$$
\left(U \delta\left(U^{*}\right)\right)^{*}=-\delta(U) U^{*}=U \delta\left(U^{*}\right)-\delta(\mathbf{1})=U \delta\left(U^{*}\right),
$$

which implies that every element in $K_{\delta}$ is self-adjoint.

Remark 3.7. Let $Z_{1}, Z_{2}, \cdots, Z_{n} \in \mathcal{Z}(\mathcal{A})$ be mutually disjoint projections such that $\delta\left(Z_{i}\right)=0$ for $i=1,2, \cdots, n$. For every $Z_{i}$, we have

$$
\begin{aligned}
K_{\delta} Z_{i} & =\overline{c o}^{w o}\left\{U \delta\left(U^{*}\right) \mid U \in \mathcal{U}(\mathcal{A})\right\} Z_{i}=\overline{c o}^{w o}\left\{U \delta\left(U^{*}\right) Z_{i} \mid U \in \mathcal{U}(\mathcal{A})\right\} \\
& =\overline{c o}^{w o}\left\{U Z_{i} \delta\left(U^{*} Z_{i}\right) \mid U \in \mathcal{U}(\mathcal{A})\right\}=\overline{c o}^{w o}\left\{U \delta\left(U^{*}\right) \mid U \in \mathcal{U}\left(\mathcal{A}_{Z_{i}}\right)\right\} .
\end{aligned}
$$

Since $\delta(\mathbf{1})=0$, it follows that $\delta\left(\mathbf{1}-\sum_{i=1}^{n} Z_{i}\right)=0$. Therefore, since $Z_{i}$ are mutually disjoint, for every $U_{1}, U_{2}, \cdots U_{n} \in \mathcal{U}(\mathcal{A})$, we have that

$$
\begin{aligned}
\sum_{i=1}^{n} U_{i} Z_{i} \delta\left(U_{i}^{*} Z_{i}\right) & =\left(\sum_{i=1}^{n} U_{i} Z_{i}\right) \delta\left(\sum_{i=1}^{n} U_{i} Z_{i}\right) \\
& =\left(\sum_{i=1}^{n} U_{i} Z_{i}+\mathbf{1}-\sum_{i=1}^{n} Z_{i}\right) \delta\left(\sum_{i=1}^{n} U_{i} Z_{i}+\mathbf{1}-\sum_{i=1}^{n} Z_{i}\right) .
\end{aligned}
$$

Note that $\sum_{i=1}^{n} U_{i} Z_{i}+\mathbf{1}-\sum_{i=1}^{n} Z_{i} \in \mathcal{U}(\mathcal{A})$. Thus, $\sum_{i=1}^{n} U_{i} Z_{i} \delta\left(U_{i}^{*} Z_{i}\right) \in K_{\delta}$. For any $X_{1} \in K_{\delta} Z_{1}$, there is a net in $c o\left\{U_{1} Z_{1} \delta\left(U_{1}^{*} Z_{1}\right)\right\}$ converging to $X_{1}$ in the weak operator topology. Hence, $X_{1} \oplus\left(\oplus_{i=2}^{n} U_{i} Z_{i} \delta\left(U_{i}^{*} Z_{i}\right)\right) \in K_{\delta}$. By mathematical induction, we obtain that $\oplus_{i=1}^{n} X_{i} \in K_{\delta}$ for any $X_{i} \in K_{\delta} Z_{i}$. That is, $\sum_{i=1}^{n} K_{\delta} Z_{i} \subset K_{\delta}$.

In the following Proposition, we provide an auxiliary result which allows us to use Lemma 2.6 in the proof of Theorem 4.2.

Proposition 3.8. Let $T \in K_{\delta}$ and let $\varepsilon, s>0$. If $0<s \leq \tau\left(E^{|T|}(\varepsilon, \infty)\right)$, then there is a unitary element $U \in \mathcal{U}(\mathcal{A})$ such that

$$
\int_{0}^{s / 2} \mu(t ; \delta(U)) d t>\frac{s}{2} \varepsilon .
$$

Proof. Since $T \in K_{\delta}$, it follows from (3.1) that there is a net $\left\{B_{\alpha}\right\}_{\alpha}$ with

$$
B_{\alpha}:=\sum_{i=1}^{n_{\alpha}} \lambda_{\alpha}^{(i)} U_{\alpha}^{(i)} \delta\left(\left(U_{\alpha}^{(i)}\right)^{*}\right), 1 \leq n_{\alpha}<\infty, U_{\alpha}^{(i)} \in \mathcal{U}(\mathcal{A}), \sum_{i=1}^{n_{\alpha}} \lambda_{\alpha}^{(i)}=1,
$$

converging to $T$ in the strong operator topology. Note that every $B_{\alpha}$ is self-adjoint. By [52, Proposition 2.3.2], we have $\left|B_{\alpha}\right| \rightarrow_{\text {so }}|T|$, and therefore, employing Lemma 2.3, 
we infer that there exists a $B_{\alpha}$ such that $\tau\left(E^{\left|B_{\alpha}\right|}(\varepsilon, \infty)\right)>\frac{s}{2}$. Hence, Lemma 2.4 implies that

$$
\mu\left(t ; \sum_{i=1}^{n_{\alpha}} \lambda_{\alpha}^{(i)} U_{\alpha}^{(i)} \delta\left(\left(U_{\alpha}^{(i)}\right)^{*}\right)\right)=\mu\left(t ; B_{\alpha}\right)>\varepsilon, \quad t \in\left[0, \frac{s}{2}\right] .
$$

Now, it follows from [47, Theorem 3.3.3] that

$$
\sum_{i=1}^{n_{\alpha}} \lambda_{\alpha}^{(i)} \int_{0}^{\frac{s}{2}} \mu\left(t ; U_{\alpha}^{(i)} \delta\left(\left(U_{\alpha}^{(i)}\right)^{*}\right)\right) d t \geq \int_{0}^{\frac{s}{2}} \mu\left(t ; \sum_{i=1}^{n_{\alpha}} \lambda_{\alpha}^{(i)} U_{\alpha}^{(i)} \delta\left(\left(U_{\alpha}^{(i)}\right)^{*}\right)\right) d t \stackrel{(3.2)}{>} \frac{s}{2} \varepsilon .
$$

Thus, there exists $U_{\alpha}^{(i)} \in \mathcal{U}(\mathcal{A})$ such that

$$
\int_{0}^{\frac{s}{2}} \mu\left(t ; \delta\left(\left(U_{\alpha}^{(i)}\right)^{*}\right)\right) d t \stackrel{(2.2)}{\geq} \int_{0}^{\frac{s}{2}} \mu\left(t ; U_{\alpha}^{(i)} \delta\left(\left(U_{\alpha}^{(i)}\right)^{*}\right)\right) d t>\frac{s}{2} \varepsilon .
$$

Recall, that our aim is to show that any derivation $\delta: \mathcal{A} \rightarrow \mathcal{C}_{0}(\mathcal{M}, \tau)$ inner. Hence, if we have a central partition of unity $\left\{Z_{i}\right\}$ of $\mathcal{A}$ such that $\delta$ is inner on every $\mathcal{A}_{Z_{i}}$ and is implemented by $K_{i} \in Z_{i} \mathcal{C}_{0}(\mathcal{M}, \tau) Z_{i}$, then a natural choice of element implementing $\delta$ on $\mathcal{A}$ is $\oplus_{i} K_{i}$. However, it can happen that $K_{i} \in Z_{i} \mathcal{C}_{0}(\mathcal{M}, \tau) Z_{i}$, but $\oplus_{i} K_{i} \notin \mathcal{C}_{0}(\mathcal{M}, \tau)$ (as an example, consider the algebra $\mathcal{M}=L_{\infty}(0, \infty)$ and partition $\left\{Z_{i}\right\}=\left\{\chi_{(i, i+1]}\right\}$ ). The latter fact is in direct contrast with $\left[55,2.11\right.$.], since if $\left\{Z_{i}\right\}_{i}$ is a central partition of the identity of $\mathcal{M}$, then the direct sum of a family of uniformly bounded operators $K_{i} \in \mathcal{J}\left(\mathcal{M}_{Z_{i}}\right)$ is also in $\mathcal{J}(\mathcal{M})$. We tackle this issue for $\tau$-compact operators, by showing that under additional assumption that every operator $K_{i}$ is chosen from $Z_{i} K_{\delta}$, the direct sum $K:=\oplus_{i} K_{i}$ is also $\tau$-compact.

Theorem 3.9. Let $\mathcal{A}$ be a von Neumann subalgebra of $\mathcal{M}$ and let $\left\{Z_{i} \in \mathcal{P}(\mathcal{A})\right\}_{i}$ be a central partition of the unity in $\mathcal{A}$. Assume that $\delta(Z)=0$ for every $Z \in \mathcal{Z}(\mathcal{A})$. If there exists $K_{i} \in \mathcal{C}_{0}\left(\mathcal{M}_{Z_{i}}, \tau\right) \cap Z_{i} K_{\delta}$ such that $\delta=\delta_{K_{i}}$ on $\mathcal{A}_{Z_{i}}$ for every $i$, then $K:=\oplus_{i} K_{i} \in \mathcal{C}_{0}(\mathcal{M}, \tau) \cap K_{\delta}$ with $\delta=\delta_{K}$ on $\mathcal{A}$.

Proof. Note that the operators $K_{i}$ and $K$ are self-adjoint. Since $\delta\left(Z_{i}\right)=0$ for every $i$, it follows that $Z_{i} \delta(X)=\delta\left(Z_{i} X Z_{i}\right)=Z_{i} \delta(X) Z_{i}=\delta(X) Z_{i}$ for every $X \in \mathcal{A}$. Hence, that fact that $\left\{Z_{i}\right\}$ is a central partition of unity, together with the assumption that $\delta=\delta_{K_{i}}$ on $\mathcal{A}_{Z_{i}}$ implies that for every $X \in \mathcal{A}$, we have

$$
\begin{aligned}
\delta(X) & =\oplus_{i}\left(Z_{i} \delta(X)\right)=\oplus_{i}\left(Z_{i} \delta(X) Z_{i}\right)=\oplus_{i} \delta\left(Z_{i} X Z_{i}\right) \\
& =\oplus_{i} \delta_{K_{i}}\left(Z_{i} X Z_{i}\right)=\oplus_{i} \delta_{K_{i}}(X)=\delta_{K}(X) .
\end{aligned}
$$

We assert that $K \in K_{\delta}$. Let $\tau_{\mathcal{Z}(\mathcal{A})}$ be a semifinite faithful normal trace $\mathcal{Z}(\mathcal{A})$. It follows that there is an increasing net $\left\{R_{\lambda}\right\} \subset\{\mathcal{Z}(\mathcal{A})\}$ of $\tau_{\mathcal{Z}(\mathcal{A})}$-finite projections such that $R_{\lambda} \uparrow \mathbf{1}$. It is a fact that the reduced von Neumann algebra $\mathcal{Z}(\mathcal{A})_{R_{\lambda}}$ is finite and countably decomposable for every $R_{\lambda}$ (see e.g. [51, Theorem 1.3.6] for a proof of this fact). Thus, for every fixed $\lambda$, there are only countably many $Z_{i}$ such that $Z_{i} R_{\lambda} \neq 0$. We denote the sequence consists of non-zero elements from $\left\{Z_{i} R_{\lambda}\right\}$ by $\left\{P_{n}\right\}_{n=1}^{\infty}$. Note that for every $k$, we have

$$
P_{k} K P_{k} \in P_{k} K_{\delta} P_{k}
$$


By Remark 3.7, $\oplus_{k=1}^{n} P_{k} K P_{k} \in K_{\delta} R_{\lambda}$ for every $n$. Since $\sum_{k=1}^{\infty} P_{k}=R_{\lambda}$, it follows that

$$
R_{\lambda} K R_{\lambda}=\oplus_{k=1}^{\infty} P_{k} K P_{k} \in K_{\delta} R_{\lambda}
$$

Since $R_{\lambda} \in \mathcal{Z}(\mathcal{A})$, by Remark 3.7 again, we have that

$$
R_{\lambda} K R_{\lambda} \in K_{\delta} R_{\lambda} \subset K_{\delta} .
$$

Since $R_{\lambda} K R_{\lambda} \rightarrow_{\text {so }} K$, we obtain that $K \in K_{\delta}$.

Now, we prove that $K$ is $\tau$-compact. If the net $\left\{Z_{i}\right\}$ consists of finitely many projections, then $K$ is clearly $\tau$-compact. We assume that $\left\{Z_{i}\right\}$ contains infinitely many projections and $K \notin \mathcal{C}_{0}(\mathcal{M}, \tau)$. By the definition of $\mathcal{C}_{0}(\mathcal{M}, \tau)$, there exists an $\varepsilon>0$ such that $\infty=\tau\left(E^{|K|}(\varepsilon, \infty)\right)=\tau\left(E^{\oplus_{i}\left|K_{i}\right|}(\varepsilon, \infty)\right)$. Noting that $\tau$ is completely additive (see e.g. [63, Chapter VII, Theorem 1.11]), we obtain that $\sum_{i} \tau\left(E^{\left|K_{i}\right|}(\varepsilon, \infty)\right)=\infty$. Hence, we can choose countably many distinct $T_{j}:=K_{i(j)}$ from $\left\{K_{i}\right\}$ such that

$$
\tau\left(E^{\oplus_{j=1}^{\infty}\left|T_{j}\right|}(\varepsilon, \infty)\right)=\sum_{j=1}^{\infty} \tau\left(E^{\left|T_{j}\right|}(\varepsilon, \infty)\right)=\sum_{j=1}^{\infty} t_{j}=\infty
$$

where $t_{j}:=\tau\left(E^{\left|T_{j}\right|}(\varepsilon, \infty)\right) \in(0, \infty), 1 \leq j<\infty$. We denote $Z_{i(j)}$ by $Q_{j}$.

Note that for every $1 \leq j<\infty$, we have

$$
T_{j} \in \overline{c o}^{s o}\left\{U \delta\left(U^{*}\right) \mid U \in \mathcal{U}\left(\mathcal{A}_{Q_{j}}\right)\right\} .
$$

For every $j$, by Proposition 3.8, we can choose a $U_{j} \in \mathcal{U}\left(\mathcal{A}_{Q_{j}}\right)$ such that

$$
\int_{0}^{\frac{t_{j}}{2}} \mu\left(t ; \delta\left(U_{j}\right)\right) d t>\frac{t_{j}}{2} \varepsilon .
$$

Let $U:=\oplus_{j=1}^{\infty} U_{j} \in \mathcal{A}$. Since $\delta$ vanishes on $\left\{Q_{j}\right\}$, it follows that $\delta\left(U_{j}\right)=\delta\left(Q_{j} U Q_{j}\right)=$ $Q_{j} \delta(U) Q_{j}$. Thus, for every $n$, we have

$$
\int_{0}^{\sum_{j=1}^{n} \frac{t_{j}}{2}} \mu(t ; \delta(U)) d t \stackrel{(2.3)}{\geq} \sum_{j=1}^{n} \int_{0}^{\frac{t_{j}}{2}} \mu\left(t ; \delta\left(U_{j}\right)\right) d t \stackrel{(3.3)}{\geq} \sum_{j=1}^{n} \frac{t_{j}}{2} \varepsilon .
$$

Noticing that $\delta(U) \in \mathcal{M}$ and recalling that $\sum_{j=1}^{\infty} t_{j}=\infty$, by Lemma 2.6, we obtain that $\delta(U)$ is not $\tau$-compact, which is a contradiction and hence $K \in \mathcal{C}_{0}(\mathcal{M}, \tau)$.

We end this section by showing a fine property of inner derivations $\delta: \mathcal{A} \rightarrow$ $\mathcal{C}_{0}(\mathcal{M}, \tau)$, which is related to the set $K_{\delta}$ (see Definition 3.5). As we show in Proposition 3.10 below, for any inner derivation $\delta: \mathcal{A} \rightarrow \mathcal{C}_{0}(\mathcal{M}, \tau)$, there exists an operator $T^{\prime} \in K_{\delta}$ implementing $\delta$. We note that analogous property for inner derivations from $\mathcal{A}$ into $\mathcal{J}(\mathcal{M})$ is established in [55], however, our approach is completely different from that used in the proof of [55, Lemma 4.6]. Furthermore, our result holds if the assumption on $\mathcal{A}$ is relaxed to a weaker assumption that $\mathcal{A}$ is a unital (that is, $\mathbf{1}_{\mathcal{A}}=\mathbf{1}_{\mathcal{M}}$ ) $C^{*}$-subalgebra of $\mathcal{M}$.

Proposition 3.10. Let $\mathcal{N}$ be a unital $C^{*}$-subalgebra of $\mathcal{M}$ and let $\delta: \mathcal{N} \rightarrow \mathcal{C}_{0}(\mathcal{M}, \tau)$ be a derivation. If there exists $T \in \mathcal{C}_{0}(\mathcal{M}, \tau)$ such that $\delta=\delta_{T}$, then there exists an element $T^{\prime} \in K_{\delta}=\overline{c o}^{\text {wo }}\left\{U \delta\left(U^{*}\right) \mid U \in \mathcal{U}(\mathcal{N})\right\}$ such that $\delta=\delta_{T^{\prime}}$. 
Proof. Let $P_{n}:=E^{|T|}\left(\frac{1}{n}, \infty\right)$ and let $T_{n}:=T P_{n}$. For every $n$, projection $P_{n}$ is $\tau$ finite and $\left\|T-T_{n}\right\|_{\infty} \leq \frac{1}{n}$. In particular, $T_{n} \in L_{2}(\mathcal{M}, \tau)$, where $L_{2}(\mathcal{M}, \tau)$ denotes the noncommutative $L_{2}$-space affiliated with $\mathcal{M}$. Hence, $\delta_{T_{n}}$ has range inside $L_{2}(\mathcal{M}, \tau) \cap \mathcal{M}$ and therefore, by [10, Theorem 3.1 and Proposition 3.4], there exists

$$
T_{n}^{\prime} \in \overline{\operatorname{co}\left\{U \delta_{T_{n}}\left(U^{*}\right) \mid U \in \mathcal{U}(\mathcal{N})\right\}^{\|\cdot\|_{2}}}={\overline{\operatorname{co}\left\{T_{n}-U T_{n} U^{*} \mid U \in \mathcal{U}(\mathcal{N})\right.}}^{\|\cdot\|_{2}}
$$

such that $\left\|T_{n}^{\prime}\right\|_{\infty} \leq\left\|\delta_{T_{n}}\right\|_{\infty} \leq 2\left\|T_{n}\right\|_{\infty} \leq 2\|T\|_{\infty}$ and $\delta_{T_{n}}=\delta_{T_{n}^{\prime}}$. Hence, by [20, Chapter IX, Proposition 5.5], there is a (wo)-cluster point $T^{\prime} \in \cap_{n}{\overline{\left\{T_{n}^{\prime}, T_{n+1}^{\prime}, \cdots\right\}}}^{\text {wo }}$ for the sequence $\left\{T_{n}^{\prime}\right\}$ in the ball of radius $2\|T\|_{\infty}$ in $\mathcal{M}$.

Since $\|\cdot\|_{2}$ induces the strong operator topology, and the strong operator closure and the weak operator closure of the convex hull of a uniformly bounded set coincide, it follows that $T_{n}^{\prime} \in \overline{c o}^{w o}\left\{T_{n}-U T_{n} U^{*} \mid U \in \mathcal{U}(\mathcal{N})\right\}$. Since $\| T_{n}-U T_{n} U^{*}-(T-$ $\left.U T U^{*}\right) \|_{\infty} \leq \frac{2}{n}$, it follows from the Kaplansky density theorem (see e.g. [62, Chapter II, Theorem 4.8]) that there is an element $B_{n} \in \overline{c o}^{w o}\left\{T-U T U^{*} \mid U \in \mathcal{U}(\mathcal{N})\right\}=K_{\delta}$ such that

$$
\left\|T_{n}^{\prime}-B_{n}\right\|_{\infty} \leq \frac{2}{n}
$$

Thus, $T^{\prime}$ is a (wo)-cluster point of $\left\{B_{n}\right\}$ and therefore $T^{\prime} \in K_{\delta}$.

For every $X \in \mathcal{N}, \eta, \xi \in \mathcal{H}$, we set $\omega(\cdot)=\langle\cdot X \eta, \xi\rangle$ and $\rho(\cdot)=\left\langle\cdot \eta, X^{*} \xi\right\rangle$ on $\mathcal{M}$. For every $\varepsilon>0$, there exists $N>2 / \varepsilon$ such that

$$
\left|\omega\left(T^{\prime}-T_{N}^{\prime}\right)\right|,\left|\rho\left(T^{\prime}-T_{N}^{\prime}\right)\right|<\varepsilon .
$$

Recall that $\delta_{T_{N}}=\delta_{T_{N}^{\prime}}$. We have

$$
\begin{aligned}
\mid\langle[ & \left.\left.-T^{\prime}, X\right] \eta, \xi\right\rangle \mid \\
\leq & \left|\left\langle\left[T-T_{N}, X\right] \eta, \xi\right\rangle\right|+\left|\left\langle\left[T_{N}-T_{N}^{\prime}, X\right] \eta, \xi\right\rangle\right|+\left|\left\langle\left[T_{N}^{\prime}-T^{\prime}, X\right] \eta, \xi\right\rangle\right| \\
& \leq\left|\left\langle\left[T-T_{N}, X\right] \eta, \xi\right\rangle\right|+\left|\left\langle\left[T_{N}^{\prime}-T^{\prime}, X\right] \eta, \xi\right\rangle\right| \\
& \left.\leq\left|\left\langle\left(T-T_{N}\right) X \eta, \xi\right)\right\rangle|+|\left\langle X\left(T-T_{N}\right) \eta, \xi\right)\right\rangle \mid \\
& +\left|\omega\left(T_{N}^{\prime}-T^{\prime}\right)\right|+\left|\rho\left(T_{N}^{\prime}-T^{\prime}\right)\right| \\
& \leq \frac{2}{N}\|X\|_{\infty}\|\eta\|_{\mathcal{H}}\|\xi\|_{\mathcal{H}}+2 \varepsilon \leq \varepsilon\|X\|_{\infty}\|\eta\|_{\mathcal{H}}\|\xi\|_{\mathcal{H}}+2 \varepsilon .
\end{aligned}
$$

Since $\varepsilon$ is arbitrary, we infer that $\left[T-T^{\prime}, X\right]=0$ for every $X \in \mathcal{N}$. Hence, $T-T^{\prime} \in \mathcal{N}^{\prime}$ and therefore $\delta=\delta_{T}=\delta_{T^{\prime}}$.

\section{The Abelian Case}

Let $\mathcal{M}$ be a von Neumann algebra equipped with a semi-finite faithful normal trace $\tau$ and let $\mathcal{C}_{0}(\mathcal{M}, \tau)$ be the ideal of all $\tau$-compact operator affiliated with $\mathcal{M}$. In this section, we consider derivations $\delta: \mathcal{A} \rightarrow \mathcal{C}_{0}(\mathcal{M}, \tau)$, where $\mathcal{A}$ is an abelian von Neumann subalgebra of $\mathcal{M}$. We show that in this case, any derivation $\delta$ is inner. In particular, this result allows us to assume in the following sections that we work with derivations vanishing on the center of the subalgebra $\mathcal{A}$ of $\mathcal{M}$.

We note that even though $\mathcal{C}_{0}(\mathcal{M}, \tau) \subset \mathcal{J}(\mathcal{M})$ and $\mathcal{C}_{0}(\mathcal{M}, \tau)$ behaves somewhat like $\mathcal{J}(\mathcal{M})$, the additional restrictions to the abelian subalgebra $\mathcal{A}$ in [44,55] are no longer 
required. Moreover, since $\mathcal{C}_{0}(\mathcal{M}, \tau)$ is not necessarily the dual space of a Banach space, the techniques used in [44, Theorem 14] are not applicable in this case.

Throughout this section, we assume that $\mathcal{A}$ is an abelian von Neumann subalgebra of $\mathcal{M}$.

Let $\delta: \mathcal{A} \rightarrow \mathcal{M}$ be a derivation. The following result is well-known (see e.g. [44, Section 3] and [37, Theorem 2.1]).

Proposition 4.1. If $\mathcal{A}$ is an abelian von Neumann subalgebra of $\mathcal{M}$, then every derivation $\delta$ from $\mathcal{A}$ into $\mathcal{M}$ is inner. That is, $\delta=\delta_{T}$ for some $T \in \mathcal{M}$.

In what follows, we consider derivations $\delta: \mathcal{A} \rightarrow \mathcal{C}_{0}(\mathcal{M}, \tau)$. Since $\mathcal{C}_{0}(\mathcal{M}, \tau) \subset \mathcal{M}$, $\delta$ is a derivation from $\mathcal{A}$ into $\mathcal{M}$ and therefore there exists an operator $T \in \mathcal{M}$ such that $\delta=\delta_{T}$. Thus, our aim in this section is to show that $T$ can be chosen to be $\tau$-compact.

Recall that an expectation $\Phi$ is a norm one projection from $B(\mathcal{H})$ onto a von Neumann algebra (see [21, Section 8]). Motivated by the idea related to an expectation from $B(\mathcal{H})$ onto $\mathcal{A}^{\prime}$ used in [21, Theorem 10.9], we prove the main theorem of this section by techniques different from those used in [44], extending the results in [44] to the case of $\mathcal{C}_{0}(\mathcal{M}, \tau)$.

Theorem 4.2. Assume that $\mathcal{A}$ is an abelian von Neumann subalgebra of $\mathcal{M}$. Every derivation $\delta: \mathcal{A} \rightarrow \mathcal{C}_{0}(\mathcal{M}, \tau)$ is inner, that is, $\delta=\delta_{K}$ for some $K \in \mathcal{C}_{0}(\mathcal{M}, \tau) \cap K_{\delta}$.

Proof. Without loss of generality, we may assume that $\delta$ is a skew-adjoint derivation from $\mathcal{A}$ into $\mathcal{M}$ (see Sect. 3). Proposition 4.1 guarantees that there exists $T \in \mathcal{M}$ such that $\delta=\delta_{T}$. In particular, we may assume that $T$ is self-adjoint (see Remark 3.1). Let $\Phi$ be an expectation from $B(\mathcal{H})$ onto $\mathcal{A}^{\prime}$ given by [21, Theorem 8.3]. By the construction of $\Phi$ (see [21, Theorem 8.3]), we have that $\Phi(T)$ belongs to the weak* operator closed convex hull of $\left\{U T U^{*}: U \in \mathcal{U}(\mathcal{A})\right\}$. In particular, $\Phi(T) \in \mathcal{M}$. Set $K:=T-\Phi(T) \in \mathcal{M}$. It is clear that $\delta=\delta_{K}$ and $K$ belongs to the weak* operator closure of the convex hull of $\left\{U \delta\left(U^{*}\right) \mid U \in \mathcal{U}(\mathcal{A})\right\}$. It suffices to prove that $K \in \mathcal{C}_{0}(\mathcal{M}, \tau)$. Since $T$ is self-adjoint, it follows that $K$ is also self-adjoint.

Assume by contradiction that $K \notin \mathcal{C}_{0}(\mathcal{M}, \tau)$, i.e., there is an $\varepsilon>0$ such that $\mu(\infty ; K)>\varepsilon$. We claim that there exists $A \in \mathcal{A}$ such that $\delta(A)$ is not $\tau$-compact. To this end, we intend to use Lemma 2.6. For convenience, we divide the proof into several steps.

(a) Let

$$
\mathcal{P}:=\{P \in \mathcal{P}(\mathcal{A}) \mid \mu(\infty ; P K P)>\varepsilon\} .
$$

We claim that there is a maximal downwards directed chain $\left\{P_{\gamma}\right\}$ of infinitely many elements in $\mathcal{P}$ which satisfies $P_{0}:=\inf \left\{P_{\gamma}\right\} \notin \mathcal{P}$ and $P_{\gamma}-P_{0} \in \mathcal{P}$ for every $\gamma$.

It is clear that $\mathcal{P}$ is not empty as $\mathbf{1} \in \mathcal{P}$. We note, in addition, that $\tau(P)=\infty$ for any $P \in \mathcal{P}$. Take an arbitrary $P \in \mathcal{P}$. Assume that $P$ is minimal in $\mathcal{A}$. The same argument in the proof of [44, Lemma 8 or Theorem 14] yields that $P T P=0$. Since $P \in \mathcal{A} \subset \mathcal{A}^{\prime}$, it follows from [21, Theorem 8.1] that

$$
P K P=P T P-P \Phi(T) P=P T P-\Phi(P T P)=0,
$$

which is a contradiction to $P \in \mathcal{P}$. Thus, $\mathcal{P}$ contains no minimal element in $\mathcal{A}$. have

Now, let $Q \in \mathcal{P}(\mathcal{A})$ be such that $0 \neq Q \supsetneqq P$ and let $Q_{1}=Q, Q_{2}=P-Q$. We

$$
\begin{aligned}
P K P & =Q_{1} K Q_{1}+Q_{2} K Q_{2}+Q_{1} K Q_{2}+Q_{2} K Q_{1} \\
& =Q_{1} K Q_{1}+Q_{2} K Q_{2}+\delta\left(Q_{1}\right) Q_{2}+\delta\left(Q_{2}\right) Q_{1},
\end{aligned}
$$


where we used the fact that $Q_{1} \perp Q_{2}$ and $\delta=\delta_{K}$ for the second equality. Since $\mu(\infty ; P K P)>\varepsilon$, [47, Corollary 2.3.16] implies that

$$
\begin{aligned}
& \mu\left(t ; Q_{1} K Q_{1}+Q_{2} K Q_{2}\right)+\mu\left(s_{1} ; \delta\left(Q_{1}\right) Q_{2}\right)+\mu\left(s_{2} ; \delta\left(Q_{2}\right) Q_{1}\right) \\
& \quad \geq \mu\left(t+s_{1}+s_{2} ; P K P\right) \geq \mu(\infty ; P K P)>\varepsilon
\end{aligned}
$$

for all $t, s_{1}, s_{2}>0$. Let $\varepsilon_{1}$ be such that $\mu(\infty ; P K P)>\varepsilon_{1}>\varepsilon$. Since $\mu\left(s_{1} ; \delta\left(Q_{1}\right) Q_{2}\right)$, $\mu\left(s_{2} ; \delta\left(Q_{2}\right) Q_{1}\right) \rightarrow 0$ as $s_{1}, s_{2} \rightarrow \infty$, we have

$$
\mu\left(t ; Q_{1} K Q_{1}+Q_{2} K Q_{2}\right)>\varepsilon_{1}>\varepsilon, t \in[0, \infty) .
$$

Assume that both projections $E^{\left|Q_{1} K Q_{1}\right|}\left(\varepsilon_{1}, \infty\right)$ and $E^{\left|Q_{2} K Q_{2}\right|}\left(\varepsilon_{1}, \infty\right)$ are $\tau$-finite. Since $Q_{1} \perp Q_{2}$, it follows that $E^{\left|Q_{1} K Q_{1}\right|}\left(\varepsilon_{1}, \infty\right)+E^{\left|Q_{2} K Q_{2}\right|}\left(\varepsilon_{1}, \infty\right)=E^{\left|Q_{1} K Q_{1}+Q_{2} K Q_{2}\right|}$ $\left(\varepsilon_{1}, \infty\right)$, and therefore

$$
\begin{aligned}
\tau\left(E^{\left|Q_{1} K Q_{1}+Q_{2} K Q_{2}\right|}\left(\varepsilon_{1}, \infty\right)\right) & =\tau\left(E^{\left|Q_{1} K Q_{1}\right|+\left|Q_{2} K Q_{2}\right|}\left(\varepsilon_{1}, \infty\right)\right) \\
& =\tau\left(E^{\left|Q_{1} K Q_{1}\right|}\left(\varepsilon_{1}, \infty\right)\right)+\tau\left(E^{\left|Q_{2} K Q_{2}\right|}\left(\varepsilon_{1}, \infty\right)\right)<\infty .
\end{aligned}
$$

By Lemma 2.4, we obtain a contradiction to (4.2). Hence, either $\tau\left(E^{\left|Q_{1} K Q_{1}\right|}\left(\varepsilon_{1}, \infty\right)\right)=$ $\infty$ or $\tau\left(E^{\left|Q_{2} K Q_{2}\right|}\left(\varepsilon_{1}, \infty\right)\right)=\infty$. By Lemma 2.4, either $\mu\left(\infty ; Q_{1} K Q_{1}\right) \geq \varepsilon_{1}>\varepsilon$ or $\mu\left(\infty ; Q_{2} K Q_{2}\right) \geq \varepsilon_{1}>\varepsilon$, which implies that either $Q_{1}$ or $Q_{2}$ belongs to $\mathcal{P}$. This shows that $\mathcal{P}$ has no minimal elements, that is, for every element $P \in \mathcal{P}$, we can always find an element $Q \in \mathcal{P}$ such that $Q \leq P$. Moreover, if $P \in \mathcal{P}$ and $Q \in \mathcal{P}(\mathcal{A})$ such that $Q \leq P$, then either $Q$ or $P-Q$ belongs to $\mathcal{P}$.

Let $\left\{P_{\gamma}\right\}$ be a maximal downwards directed chain in $\mathcal{P}$ and let $P_{0}=\inf \left\{P_{\gamma}\right\}$. Obviously, $P_{0} \notin \mathcal{P}$. Otherwise, there exists a $P \supsetneqq P_{0}$ with $P \in \mathcal{P}$, which contradicts the maximality of $\left\{P_{\gamma}\right\}$. By the property stated in the above paragraph, either $P_{\gamma}-P_{0}$ or $P_{0}$ must belong to $\mathcal{P}$. However, $P_{0} \notin \mathcal{P}$. Thus, $P_{\gamma}-P_{0} \in \mathcal{P}$.

(b) Now, let us construct a sequence $\gamma_{1} \succ \gamma_{2} \succ \cdots$ such that the projection $Q_{k}:=$ $P_{\gamma_{k}}-P_{\gamma_{k+1}}$ satisfies

$$
\mu\left(t ; Q_{k} K Q_{k}\right)>\varepsilon, t \in[0,2] .
$$

Take an arbitrary $\gamma$ and set $\gamma_{1}=\gamma$. Assume that the sequence $\gamma_{1} \prec \gamma_{2} \prec \cdots \prec \gamma_{n}$ is constructed for some $n \in \mathbb{N}$. Let $A_{n}:=\left(P_{\gamma_{n}}-P_{0}\right) K\left(P_{\gamma_{n}}-P_{0}\right)$. We have that $A_{n}^{*}=A_{n}$. Furthermore, since $P_{\gamma}-P_{0} \in \mathcal{P}$, it follows that $\mu\left(\infty ; A_{n}\right)>\varepsilon$, which guarantees that $\tau\left(E^{\left|A_{n}\right|}(\varepsilon, \infty)\right)=\infty$ (see Lemma 2.4). Since $\mathbf{1}-P_{\gamma}+P_{0} \uparrow \mathbf{1}$, it follows from [52, Proposition 2.3.2] that

$$
\text { so }-\lim _{\gamma}\left|\left(\mathbf{1}-P_{\gamma}+P_{0}\right) A_{n}\left(\mathbf{1}-P_{\gamma}+P_{0}\right)\right|=\left|A_{n}\right| .
$$

Then, by Lemma 2.3, we have

$$
\liminf _{\gamma} \tau\left(E^{\left|\left(\mathbf{1}-P_{\gamma}+P_{0}\right) A_{n}\left(\mathbf{1}-P_{\gamma}+P_{0}\right)\right|}(\varepsilon, \infty)\right) \geq \tau\left(E^{\left|A_{n}\right|}(\varepsilon, \infty)\right)=\infty .
$$

Hence, we can find $\gamma_{n+1} \succ \gamma_{n}$ such that $\tau\left(E^{\left|\left(\mathbf{1}-P_{\gamma_{n+1}}+P_{0}\right) A_{n}\left(\mathbf{1}-P_{\gamma_{n+1}}+P_{0}\right)\right|}(\varepsilon, \infty)\right)>2$, and therefore, by Lemma 2.4, we have

$$
\mu\left(t ;\left(\mathbf{1}-P_{\gamma_{n+1}}+P_{0}\right) A_{n}\left(\mathbf{1}-P_{\gamma_{n+1}}+P_{0}\right)\right)>\varepsilon, t \in[0,2] .
$$

Since $P_{\gamma} \downarrow$, it follows that $\left(P_{\gamma_{n}}-P_{0}\right)\left(\mathbf{1}-P_{\gamma_{n+1}}+P_{0}\right)=\left(P_{\gamma_{n}}-P_{\gamma_{n+1}}\right)$, which implies that, setting $Q_{n}:=P_{\gamma_{n}}-P_{\gamma_{n+1}}$, we obtain that $\mu\left(t ; Q_{n} K Q_{n}\right)>\varepsilon$ for all $t \in[0,2]$. 
(c) We claim that for every $k \in \mathbb{N}$, there is a $U_{k} \in \mathcal{U}(\mathcal{A})$ such that

$$
\int_{0}^{1} \mu\left(t ; Q_{k} \delta\left(U_{k}\right) Q_{k}\right) d t>\varepsilon
$$

Since $\Phi(K)=\Phi(T-\Phi(T))=0$, by [21, Theorem 8.3], the operator

$$
Q_{k} K Q_{k}=Q_{k}(K-\Phi(K)) Q_{k}
$$

belongs to the weak* operator closure of

$$
\operatorname{co}\left\{Q_{k}\left(K-U K U^{*}\right) Q_{k}: U \in \mathcal{U}(\mathcal{A})\right\}=\operatorname{co}\left\{Q_{k} U \delta\left(U^{*}\right) Q_{k}: U \in \mathcal{U}(\mathcal{A})\right\} .
$$

Since $\mathcal{A}$ is abelian, it follows that $Q_{k} \delta(\cdot) Q_{k}$ is a derivation from $\mathcal{A}$ into $\mathcal{C}_{0}(\mathcal{M}, \tau)$ (see Sect. 3). Thus, $Q_{k} K Q_{k} \in K_{Q_{k} \delta(\cdot) Q_{k}}$. By the construction of $Q_{k}$ and Proposition 3.8, we conclude that $U_{k} \in \mathcal{U}(\mathcal{A})$ satisfying (4.5) exists.

(d) Finally, since $Q_{k}, U_{k} \in \mathcal{A}, Q_{i} \perp Q_{j}(i \neq j)$ and $\mathcal{A}$ is abelian, the series $\sum_{k=1}^{\infty} Q_{k} U_{k}$ converges in $\mathcal{A}$ in the strong operator topology. We define

$$
A:=\sum_{k=1}^{\infty} Q_{k} U_{k}
$$

Since $\mathcal{A}$ is abelian, it follows that

$$
Q_{k} \delta\left(Q_{k}^{\perp} X\right) Q_{k}=Q_{k} \delta\left(Q_{k}^{\perp} X Q_{k}^{\perp}\right) Q_{k}=Q_{k} Q_{k}^{\perp} X \delta\left(Q_{k}^{\perp}\right) Q_{k}+Q_{k} \delta\left(Q_{k}^{\perp} X\right) Q_{k}^{\perp} Q_{k}=0
$$

for every $X \in \mathcal{A}$, and therefore,

$$
\begin{aligned}
Q_{k} \delta(A) Q_{k} & =Q_{k} \delta\left(Q_{k} A\right) Q_{k}+Q_{k} \delta\left(Q_{k}^{\perp} A\right) Q_{k} \\
& =Q_{k} \delta\left(Q_{k} U_{k}\right) Q_{k} \\
& =Q_{k} \delta\left(Q_{k} U_{k}\right) Q_{k}+Q_{k} \delta\left(Q_{k}^{\perp} U_{k}\right) Q_{k} \\
& =Q_{k} \delta\left(U_{k}\right) Q_{k} .
\end{aligned}
$$

Hence, we obtain

$$
\int_{0}^{1} \mu\left(t ; Q_{k} \delta(A) Q_{k}\right) d t \stackrel{(4.6)}{=} \int_{0}^{1} \mu\left(t ; Q_{k} \delta\left(U_{k}\right) Q_{k}\right) d t \stackrel{(4.5)}{>} \varepsilon .
$$

Take an arbitrary $n \geq 1$. Since $Q_{i} \perp Q_{j}$ for $i \neq j$, it follows that

$$
\int_{0}^{n} \mu(t ; \delta(A)) d t \stackrel{(2.3)}{\geq} \sum_{i=1}^{n} \int_{0}^{1} \mu\left(t ; Q_{i} \delta(A) Q_{i}\right) d t \stackrel{(4.7)}{>} n \cdot \varepsilon .
$$

Now, by Lemma 2.6, we obtain that $\delta(A)$ is not $\tau$-compact, which is a contradiction. It completes the proof.

Remark 4.3. Note that the so-called locally compatible condition on the abelian von Neumann subalgebra $\mathcal{A}$ of $\mathcal{M}$ is required in studying derivations from $\mathcal{A}$ into $\mathcal{J}(\mathcal{M})$ (see [55, Proposition 4.3], see also [44]). When this condition is not fulfilled, derivations from $\mathcal{A}$ into $\mathcal{J}(\mathcal{M})$ are not necessarily inner (see [55, Theorem 1.2]). However, the "locally compatible" condition is redundant in our present setting, that is, the result of Theorem 4.2 holds without any additional assumption on the abelian subalgebra $\mathcal{A}$ of $\mathcal{M}$. 
Remark 4.4. Assume that $\mathcal{A}$ is a von Neumann subalgebra of $\mathcal{M}$. By Theorem 4.2, for every derivation $\delta: \mathcal{A} \rightarrow \mathcal{C}_{0}(\mathcal{M}, \tau),\left.\delta\right|_{\mathcal{Z}(\mathcal{A})}$ is implemented by a $\tau$-compact operator $K$. Hence, in the study of derivations $\delta: \mathcal{A} \rightarrow \mathcal{C}_{0}(\mathcal{M}, \tau)$, we can consider linear mapping $\delta-\delta_{K}$ which is a derivation from $\mathcal{A}$ into $\mathcal{C}_{0}(\mathcal{M}, \tau)$ vanishing on $\mathcal{Z}(\mathcal{A})$. That is, without loss of generality, we may assume that derivation $\delta$ vanishes on $\mathcal{Z}(\mathcal{A})$.

\section{The Properly Infinite Case}

Let, as before, $\mathcal{M}$ be a semifinite von Neumann algebra with a faithful semifinite normal trace $\tau$, and let $\mathcal{A}$ be a properly infinite von Neumann subalgebra of $\mathcal{M}$. Derivations acting on a properly infinite von Neumann algebra have been actively investigated. Christensen [15, Corollary 5.6] showed that every derivation from $\mathcal{A}$ into $B(\mathcal{H})$ is inner. It is proved by Christensen, Effros and Sinclair [17, Corollary 5.5] that every derivation $\delta$ from $\mathcal{A}$ into $\mathcal{M}$ is inner if $\mathcal{M}$ is injective (see also [15, Corollary 5.6]). Recently, in [9], Ber, Chilin and Sukochev proved that every derivation $\delta$ from $\mathcal{A}$ into a Banach $\mathcal{A}$ bimodule of locally measurable operators affiliated with $\mathcal{A}$ is inner (see also [5, Theorem 4.7]).

In this section, we show that any derivation $\delta$ on a properly infinite von Neumann subalgebra $\mathcal{A}$ of $\mathcal{M}$ with values in the ideal $\mathcal{C}_{0}(\mathcal{M}, \tau)$ is necessarily inner. We note, that the result for derivations from an abelian subalgebra of $\mathcal{M}$ (see Theorem 4.2) allows us to use the same approach for properly infinite algebras as in [44] (see also [37] and [21]).

Recall that if $\mathcal{A}$ is properly infinite von Neumann subalgebra of a semifinite von Neumann algebra $\mathcal{M}$, then there is an infinite countable decomposition of the identity into mutually orthogonal projections of $\mathcal{A}$, all equivalent in $\mathcal{A}$ to $\mathbf{1}$, and thus a fortiori equivalent in $\mathcal{M}$ to $\mathbf{1}$ [22, Part III, Chapter 8, Section 6, Corollary 2] (see also [42]).

Let $H_{0}=\ell^{2}(\mathbb{Z})$. By [22, Part I, Section 2.4, Proposition 5], there is a spatial isomorphism

$$
\phi: \mathcal{M} \rightarrow \tilde{\mathcal{M}}=\mathcal{M} \otimes B\left(H_{0}\right)
$$

with

$$
\phi(\mathcal{A})=\tilde{\mathcal{A}}=\mathcal{A} \otimes B\left(H_{0}\right) .
$$

It is well-known [22, Section 1.5, Proposition 8 ] that a spatial isomorphism is isometric and is normal, i.e., for every bounded increasing net $\left\{X_{i} \in \mathcal{M}_{+}\right\}_{i}$ satisfying $X_{i} \uparrow X$, we have $\phi\left(X_{i}\right) \uparrow \phi(X)$. Recall also that the elements $B \in \tilde{\mathcal{M}}$ (or $\tilde{\mathcal{A}}$ ) are represented by matrices $\left[B_{i j}\right], i, j \in \mathbb{Z}$, with entries in $\mathcal{M}$ (or $\mathcal{A}$ ) by the formula

$$
\left(1 \otimes E_{i j}\right) B\left(1 \otimes E_{k l}\right)=B_{j k} \otimes E_{i l},
$$

where $E_{i j}$ is the canonical matrix unit of $B\left(H_{0}\right)$. In particular, if $\mathcal{L}$ (respectively, $\mathcal{D}$ ) is the maximal abelian subalgebras of $B\left(H_{0}\right)$ of Laurent (respectively, diagonal) matrices, then $B \in \mathcal{M} \otimes \mathcal{L}$ (respectively, $B \in \mathcal{M} \otimes \mathcal{D}$ ) if and only if [ $\left.B_{i j}\right]$ is a Laurent (respectively, a diagonal) matrix with entries in $\mathcal{M}$, i.e., $B_{i j}=B_{i-j}$ (respectively, $B_{i j}=\delta_{i j} B_{i i}$, where $\delta_{i j}$ stands for the Kronecker Delta), $i, j \in \mathbb{Z}$, where $B_{k}$ denotes the entry along the $k$ th diagonal for all $k \in \mathbb{Z}$.

Let $\tau_{0}$ be the standard trace on $B\left(H_{0}\right)$ and $\tilde{\tau}:=\tau \otimes \tau_{0}$. For the properties of tensor products of von Neumann algebras, we refer the reader to [63, Chapter IV]. It is wellknown that the isomorphism $\phi$ introduced in (5.1) is trace-preserving. 
Before we proceed to the proof of the main result of this section (see Theorem 5.3 below), we establish several properties of the isomorphism $\phi$ introduced in (5.1) related to the generalised singular value functions and $\tau$-compact operators.

Proposition 5.1. Let $\phi$ be the spatial isomorphism from $\mathcal{M}$ onto $\tilde{\mathcal{M}}$ introduced in (5.1). Then, for any $X \in \mathcal{M}$, we have

(i). $\mu(X)=\mu(\phi(X))$.

(ii). $\mu(X)=\mu\left(X \otimes E_{00}\right)$.

Proof. (i). Since $\phi$ is an isometric, trace-preserving isomorphism from $\mathcal{M}$ onto $\tilde{\mathcal{M}}$, it follows from the definition of generalised singular value function (see Definition 2.1) that

$$
\begin{aligned}
\mu(t ; X) & =\inf \left\{\|X P\|_{\infty}: P \in \mathcal{P}(\mathcal{M}), \tau(\mathbf{1}-P) \leq t\right\} \\
& =\inf \left\{\|\phi(X) \phi(P)\|_{\infty}: P \in \mathcal{P}(\mathcal{M}), \tau(\mathbf{1}-P) \leq t\right\} \\
& =\inf \left\{\|\phi(X) \tilde{P}\|_{\infty}: \tilde{P} \in \mathcal{P}(\tilde{\mathcal{M}}), \tilde{\tau}(\mathbf{1}-\tilde{P}) \leq t\right\}=\mu(t ; \phi(X)) .
\end{aligned}
$$

(ii). For every $t>0$, we have

$$
\begin{aligned}
d_{\left|X \otimes E_{00}\right|}(t) & =\tilde{\tau}\left(E^{\left|X \otimes E_{00}\right|}(t, \infty)\right) \\
& =\tilde{\tau}\left(E^{|X| \otimes E_{00}}(t, \infty)\right) \\
& =\tilde{\tau}\left(E^{|X|}(t, \infty) \otimes E_{00}\right) \\
& =\tau\left(E^{|X|}(t, \infty)\right)=d_{|X|}(t) .
\end{aligned}
$$

Thus, by (2.1), we have $\mu\left(t ; X \otimes E_{00}\right)=\mu(t ; X)$.

Proposition 5.2. Let $\phi$ be the spatial isomorphism from $\mathcal{M}$ onto $\tilde{\mathcal{M}}$ as in (5.1). We have that

(i). $\mathcal{C}_{0}(\tilde{\mathcal{M}}, \tilde{\tau})=\phi\left(\mathcal{C}_{0}(\mathcal{M}, \tau)\right)$;

(ii). If $K \otimes E_{00} \in \mathcal{C}_{0}(\tilde{\mathcal{M}}, \tilde{\tau})$, then $K \in \mathcal{C}_{0}(\mathcal{M}, \tau)$;

(iii). $(\mathcal{M} \otimes \mathcal{L}) \cap \mathcal{C}_{0}(\tilde{\mathcal{M}}, \tilde{\tau})=\{0\}$.

Proof. Part (i) immediately follows from Proposition 5.1.

(ii). Suppose that $K \otimes E_{00} \in \mathcal{C}_{0}(\tilde{\mathcal{M}}, \tilde{\tau})$. On one hand, part (i) guarantees that $\phi^{-1}(K \otimes$ $\left.E_{00}\right) \in \mathcal{C}_{0}(\mathcal{M}, \tau)$. On the other hand, by Proposition 5.1, we have that $\mu\left(\phi^{-1}(K \otimes\right.$ $\left.\left.E_{00}\right)\right)=\mu(K)$. Hence, we conclude that $K \in \mathcal{C}_{0}(\mathcal{M}, \tau)$.

(iii). Let $\mathcal{J}(\tilde{\mathcal{M}})$ be the norm closure of the linear space of all finite projections of $\tilde{\mathcal{M}}$. Since $\mathcal{C}_{0}(\tilde{\mathcal{M}}, \tilde{\tau}) \subset \mathcal{J}(\tilde{\mathcal{M}})$ (see Sect. 2), it follows from [44, Lemma 12 (b)] that $(\mathcal{M} \otimes \mathcal{L}) \cap \mathcal{C}_{0}(\tilde{\mathcal{M}}, \tilde{\tau})=\{0\}$

The lifting technique used in [44] (see also [21,37]) and the already proven abelian case play crucial roles in proving Theorem 5.3. However, we can simplify the proof since the condition that $\mathcal{A}$ contains the center of $\mathcal{M}$ imposed in [44, Theorem 4] is not required in Theorem 4.2.

Theorem 5.3. Let $\mathcal{A}$ be a properly infinite von Neumann subalgebra of $\mathcal{M}$. For every derivation $\delta: \mathcal{A} \rightarrow \mathcal{C}_{0}(\mathcal{M}, \tau)$, there exists $T \in \mathcal{C}_{0}(\mathcal{M}, \tau) \cap K_{\delta}$ such that $\delta=\delta_{T}$ on $\mathcal{A}$. 
Proof. Let $\tilde{\delta}=\phi \circ \delta \circ \phi^{-1}$, where $\phi$ is a spatial isomorphism as in (5.1). Clearly, $\tilde{\delta}$ is also a derivation, and, by Proposition 5.2, we have that

$$
\tilde{\delta}: \tilde{\mathcal{A}} \rightarrow \phi\left(\mathcal{C}_{0}(\mathcal{M}, \tau)\right)=\mathcal{C}_{0}(\tilde{\mathcal{M}}, \tilde{\tau}) .
$$

Let us define the following von Neumann algebras:

$$
\tilde{\mathcal{A}}_{1}=\mathbf{1} \otimes \mathcal{L}, \quad \mathcal{A}_{1}=\phi^{-1}\left(\tilde{\mathcal{A}}_{1}\right), \quad \tilde{\mathcal{A}}_{2}=\mathcal{A} \otimes \mathcal{L} \quad \text { and } \quad \tilde{\mathcal{A}}_{3}=\mathcal{A}_{1} \otimes \mathcal{D} .
$$

By Proposition 5.2 (iii) and [62, Chapter IV, Theorem 5.9 and Corollary 5.10], we have

$$
\begin{aligned}
\tilde{\mathcal{A}}_{1}^{\prime} \cap \mathcal{C}_{0}(\tilde{\mathcal{M}}, \tilde{\tau}) & =(\mathbf{1} \otimes \mathcal{L})^{\prime} \cap\left(\left(\mathcal{M} \otimes B\left(H_{0}\right) \cap \mathcal{C}_{0}(\tilde{\mathcal{M}}, \tilde{\tau})\right)\right. \\
& =\left(\mathcal{M} \otimes \mathcal{L}^{\prime}\right) \cap \mathcal{C}_{0}(\tilde{\mathcal{M}}, \tilde{\tau}) \\
& =(\mathcal{M} \otimes \mathcal{L}) \cap \mathcal{C}_{0}(\tilde{\mathcal{M}}, \tilde{\tau}) \\
& =\{0\} .
\end{aligned}
$$

Since the isomorphism $\phi$ is spatial, we infer that

$$
\mathcal{A}_{1}^{\prime} \cap \mathcal{C}_{0}(\mathcal{M}, \tau)=\phi^{-1}\left(\tilde{\mathcal{A}}_{1}^{\prime}\right) \cap \mathcal{C}_{0}(\mathcal{M}, \tau)=\phi^{-1}\left(\tilde{\mathcal{A}}_{1}^{\prime} \cap \mathcal{C}_{0}(\tilde{\mathcal{M}}, \tilde{\tau})\right)=\{0\}
$$

We now study derivation $\tilde{\delta}$ on each of the algebras $\tilde{\mathcal{A}}_{j}, j=1,2,3$, separately.

Since $\tilde{\mathcal{A}}_{1}$ is abelian, Theorem 4.2 applied to the derivation $\left.\tilde{\delta}\right|_{\tilde{\mathcal{A}}_{1}}$ guarantees the existence of $T_{1} \in \mathcal{C}_{0}(\tilde{\mathcal{M}}, \tilde{\tau})$ such that

$$
\tilde{\delta}_{1}:=\tilde{\delta}-\delta_{T_{1}}
$$

vanishes on $\tilde{\mathcal{A}}_{1}$. Moreover, $T_{1} \in \overline{c o}^{w o}\left\{U \tilde{\delta}\left(U^{*}\right) \mid U \in \mathcal{U}\left(\tilde{\mathcal{A}}_{1}\right)\right\}$.

Note that $\tilde{\mathcal{A}}_{2}=\mathcal{A} \otimes \mathcal{L} \subset \mathcal{M} \otimes \mathcal{L} \subset \mathbf{1}^{\prime} \otimes \mathcal{L}=\tilde{\mathcal{A}}_{1}^{\prime}$. For any $A_{1} \in \tilde{A}_{1}$ and $A_{2} \in \tilde{A}_{2}$, we have

$$
A_{1} \tilde{\delta}_{1}\left(A_{2}\right)=\tilde{\delta}_{1}\left(A_{1} A_{2}\right)=\tilde{\delta}_{1}\left(A_{2} A_{1}\right)=\tilde{\delta}_{1}\left(A_{2}\right) A_{1},
$$

that is, $\tilde{\delta}_{1}\left(A_{2}\right) \in \tilde{\mathcal{A}}_{1}^{\prime}$. Therefore, it follows from (5.2) that

$$
\tilde{\delta}_{1}\left(\tilde{\mathcal{A}}_{2}\right) \subset \tilde{\mathcal{A}}_{1}^{\prime} \cap \mathcal{C}_{0}(\tilde{\mathcal{M}}, \tilde{\tau})=\{0\}
$$

which implies that the derivation $\tilde{\delta}_{1}$ also vanishes on $\tilde{\mathcal{A}}_{2}$.

Next, we consider $\tilde{\delta}_{1}$ on the algebra $\tilde{\mathcal{A}}_{3}$. Since $\tilde{\mathcal{A}}_{1}$ is abelian, it follows that $\mathcal{A}_{1}$ is also abelian and therefore, $\tilde{\mathcal{A}}_{3}$ is also abelian. Thus, we can apply Theorem 4.2 to the derivation $\left.\tilde{\delta}_{1}\right|_{\tilde{\mathcal{A}}_{3}}$ to infer that there is a $T_{2} \in \mathcal{C}_{0}(\tilde{\mathcal{M}}, \tilde{\tau})$ such that $\tilde{\delta}_{1}=\delta_{T_{2}}$ on $\tilde{\mathcal{A}}_{3}$. We claim that $T_{2}=0$, that is, $\tilde{\delta}_{1}$ vanishes on $\tilde{\mathcal{A}}_{3}$.

Since $\mathcal{A}_{1} \otimes \mathbf{1} \subset \mathcal{A} \otimes \mathbf{1} \subset \mathcal{A} \otimes \mathcal{L}=\tilde{\mathcal{A}}_{2}, \mathcal{A}_{1} \otimes \mathbf{1} \subset \tilde{\mathcal{A}}_{3}$ and $\tilde{\delta}_{1}$ vanishes on $\tilde{\mathcal{A}}_{2}$, we have $\delta_{T_{2}}$ vanishes on $\mathcal{A}_{1} \otimes \mathbf{1}$, i.e.,

$$
T_{2} \in\left(\mathcal{A}_{1} \otimes \mathbf{1}\right)^{\prime} \cap \mathcal{C}_{0}(\tilde{\mathcal{M}}, \tilde{\tau})=\left(\mathcal{A}_{1}^{\prime} \otimes B\left(H_{0}\right)\right) \cap \mathcal{C}_{0}(\tilde{\mathcal{M}}, \tilde{\tau})
$$

Hence, for all $i, j \in \mathbb{Z}$, we have that $\left(T_{2}\right)_{i j} \in \mathcal{A}_{1}^{\prime}$ and

$$
\left(T_{2}\right)_{i j} \otimes E_{00}=\left(\mathbf{1} \otimes E_{0 i}\right) T_{2}\left(\mathbf{1} \otimes E_{j 0}\right) \in \mathcal{C}_{0}(\tilde{\mathcal{M}}, \tilde{\tau}) .
$$


By Proposition 5.2 (ii), the latter condition implies that $\left(T_{2}\right)_{i j} \in \mathcal{C}_{0}(\mathcal{M}, \tau)$ and therefore, $\left(T_{2}\right)_{i j} \in \mathcal{A}_{1}^{\prime} \cap \mathcal{C}_{0}(\mathcal{M}, \tau)$ for all $i, j \in \mathbb{Z}$. Appealing to (5.3), we conclude that $\left(T_{2}\right)_{i j}=0$ for all $i, j \in \mathbb{Z}$, so $T_{2}=0$. Thus, the derivation $\tilde{\delta}_{1}$ vanishes on $\tilde{\mathcal{A}}_{3}$. In particular, $\tilde{\delta}_{1}$ vanishes on $\mathbf{1} \otimes \mathcal{D}$.

Finally, we claim that $\tilde{\delta}_{1}$ vanishes on $\tilde{\mathcal{A}}$, which would imply that $\tilde{\delta}=\delta_{T_{1}}$. Since $\mathcal{L}$ and $\mathcal{D}$ generate $B\left(H_{0}\right)$ in weak* operator topology, we have $\tilde{\mathcal{A}}_{2}=\mathcal{A} \otimes \mathcal{L}$ and $\mathbf{1} \otimes \mathcal{D}$ generate $\tilde{\mathcal{A}}$ in weak* operator topology. Since $\tilde{\delta}$ is weak* topology continuous (see [37, Lemma 1.3]), it follows that $\tilde{\delta}_{1}=\tilde{\delta}-\delta_{T_{1}}=0$, i.e., $\tilde{\delta}=\delta_{T_{1}}$ on $\tilde{\mathcal{A}}$. Then, for every $X \in \tilde{\mathcal{A}}$, we have

$$
\phi\left(\delta\left(\phi^{-1}(X)\right)\right)=\tilde{\delta}(X)=\delta_{T_{1}}(X)=X T_{1}-T_{1} X
$$

and therefore

$$
\delta\left(\phi^{-1}(X)\right)=\phi^{-1}\left(X T_{1}-T_{1} X\right)=\phi^{-1}(X) \phi^{-1}\left(T_{1}\right)-\phi^{-1}\left(T_{1}\right) \phi^{-1}(X) .
$$

Since $\phi$ is a isomorphism from $\mathcal{A}$ onto $\tilde{\mathcal{A}},(5.4)$ implies that $\delta(Y)=\delta_{\phi^{-1}\left(T_{1}\right)}(Y)$ for every $Y \in \mathcal{A}$. Since $T_{1} \in \mathcal{C}_{0}(\tilde{\mathcal{M}}, \tilde{\tau}) \cap \overline{c o}^{w o}\left\{U \tilde{\delta}\left(U^{*}\right) \mid U \in \mathcal{U}\left(\tilde{\mathcal{A}}_{1}\right)\right\}$, we have that $\phi^{-1}\left(T_{1}\right) \in \mathcal{C}_{0}(\mathcal{M}, \tau)$ and $\phi^{-1}\left(T_{1}\right) \in \overline{c o}^{w o}\left\{U \delta\left(U^{*}\right) \mid U \in \mathcal{U}\left(\mathcal{A}_{1}\right)\right\} \subset K_{\delta}$, which completes the proof.

\section{The Type I Case}

Let $\mathcal{M}$ be a semifinite von Neumann algebra equipped with a faithful semifinite normal trace $\tau$. Derivations from a type $I$ von Neumann subalgebra into $K(\mathcal{H})$ and $\mathcal{J}(\mathcal{M})$ are studied in [37] (see also [21, Section 10]) and [55], respectively. In what follows, we consider the case of derivations with values in $\mathcal{C}_{0}(\mathcal{M}, \tau)$. Even though $\mathcal{C}_{0}(\mathcal{M}, \tau)$ and $\mathcal{J}(\mathcal{M})$ are similar in many respects and $\mathcal{J}(\mathcal{M})=\mathcal{C}_{0}(\mathcal{M}, \operatorname{Tr})=K(\mathcal{H})$ when $\mathcal{M}=B(\mathcal{H})$ and $\operatorname{Tr}$ is the standard trace on $B(\mathcal{H})$, the so-called locally compatible condition imposed in [55, Theorem 1.1] is redundant in the present setting. That is, we can consider the case when $\mathcal{A}$ is an arbitrary type $I$ von Neumann subalgebra of $\mathcal{M}$. Before we proceed to the proof for the type $I$ case, we need the following proposition.

Proposition 6.1. Let $\mathcal{A}$ be a type $I_{n}$ von Neumann subalgebra of $\mathcal{M}, n \in \mathbb{N}$. Then, every derivation $\delta$ from $\mathcal{A}$ into $\mathcal{C}_{0}(\mathcal{M}, \tau)$ is inner, i.e., $\delta=\delta_{E}$ for some $E \in \mathcal{C}_{0}(\mathcal{M}, \tau)$. Moreover, $E \in \operatorname{co}\left\{U \delta\left(U^{*}\right) \mid U \in \mathcal{U}(\mathcal{A})\right\}$.

Proof. By [59, Theorem 2.3.3], we have $\mathcal{A}=M_{n} \otimes \mathcal{Z}(\mathcal{A})$, where $M_{n}$ stands for the algebra of all $n \times n$ matrices. For the sake of convenience, we denote $\mathcal{A}=M_{n} \otimes \mathcal{Z}(\mathcal{A})$ by $M_{n}(\mathcal{Z}(\mathcal{A}))$, and $E_{i j} \otimes \mathbf{1}_{\mathcal{A}}$ by $B_{i j}$, where $E_{i j}$ is the standard matrix units of $M_{n}$. In particular, every $A \in \mathcal{A}$ is in the form of $\sum_{i, j=1}^{n} A_{i j} B_{i j}, A_{i j} \in \mathcal{Z}(\mathcal{A})$.

We define

$$
D_{1}=\sum_{i=1}^{n} B_{i 1} \delta\left(B_{1 i}\right) .
$$

Since every $\delta\left(B_{1 i}\right)$ is $\tau$-compact, it follows that $D_{1}$ is a $\tau$-compact operator. 
Equality $\delta\left(\mathbf{1}_{\mathcal{A}}\right)=0$ together with the Leibniz rule implies that

$$
\begin{aligned}
D_{1} & =\sum_{i=1}^{n}\left(\delta\left(B_{i 1} B_{1 i}\right)-\delta\left(B_{i 1}\right) B_{1 i}\right)=\sum_{i=1}^{n}\left(\delta\left(B_{i i}\right)-\delta\left(B_{i 1}\right) B_{1 i}\right) \\
& =\delta\left(\mathbf{1}_{\mathcal{A}}\right)-\sum_{i=1}^{n} \delta\left(B_{i 1}\right) B_{1 i}=-\sum_{i=1}^{n} \delta\left(B_{i 1}\right) B_{1 i} .
\end{aligned}
$$

Then, for every $k, l=1, \ldots, n$ we have

$$
\begin{aligned}
{\left[B_{k l}, D_{1}\right] } & =B_{k l} D_{1}-D_{1} B_{k l} \stackrel{(6.1)}{=} B_{k l} \sum_{i=1}^{n} B_{i 1} \delta\left(B_{1 i}\right)+\left(\sum_{i=1}^{n} \delta\left(B_{i 1}\right) B_{1 i}\right) B_{k l} \\
& =B_{k 1} \delta\left(B_{1 l}\right)+\delta\left(B_{k 1}\right) B_{1 l}=\delta\left(B_{k 1} B_{1 l}\right)=\delta\left(B_{k l}\right) .
\end{aligned}
$$

Now, consider $X=\sum_{i, j=1}^{n} X_{i j} B_{i j} \in \mathcal{A}, X_{i j} \in \mathcal{Z}(\mathcal{A})$. Since $\sum_{k=1}^{n} X_{i j} B_{k k} \in \mathcal{Z}(\mathcal{A})$, we have that $\delta\left(\sum_{k=1}^{n} X_{i j} B_{k k}\right)=0$ (see Remark 4.4). Hence, using the Leibniz rule, we write

$$
\begin{aligned}
\delta(X) & =\sum_{i, j=1}^{n} \delta\left(X_{i j} B_{i j}\right)=\sum_{i, j=1}^{n} \delta\left(\left(\sum_{k=1}^{n} X_{i j} B_{k k}\right) B_{i j}\right) \\
& =\sum_{i, j=1}^{n} \delta\left(\sum_{k=1}^{n} X_{i j} B_{k k}\right) B_{i j}+\sum_{i, j=1}^{n}\left(\sum_{k=1}^{n} X_{i j} B_{k k}\right) \delta\left(B_{i j}\right) \\
& =\sum_{i, j=1}^{n}\left(\sum_{k=1}^{n} X_{i j} B_{k k}\right) \delta\left(B_{i j}\right) .
\end{aligned}
$$

Therefore, referring to (6.2), we obtain that

$$
\delta(X)=\sum_{i, j=1}^{n}\left(\sum_{k=1}^{n} X_{i j} B_{k k}\right)\left[B_{i j}, D_{1}\right] .
$$

Since $\sum_{k=1}^{n} X_{i j} B_{k k} \in \mathcal{Z}(\mathcal{A})$ and $\delta(\mathcal{Z}(\mathcal{A}))=0$, it follows from the definition of $D_{1}$ that $\sum_{k=1}^{n} X_{i j} B_{k k}$ commutes with $D_{1}$. Hence, we obtain that

$$
\delta(X)=\sum_{i, j=1}^{n}\left(\sum_{k=1}^{n} X_{i j} B_{k k}\right)\left[B_{i j}, D_{1}\right]=\sum_{i, j=1}^{n}\left[X_{i j} B_{i j}, D_{1}\right]=\left[X, D_{1}\right] .
$$

Arguing similarly, one can show that $D_{j}:=\sum_{i=1}^{n} B_{i j} \delta\left(B_{j i}\right)$ such that $\delta=\delta_{D_{j}}$ for every $j$. Define

$$
E:=\frac{1}{n} \sum_{j=1}^{n} D_{j}=\frac{1}{n} \sum_{i, j} B_{i j} \delta\left(B_{j i}\right)
$$

Then, $\delta=\frac{1}{n} \sum_{j=1}^{n} \delta_{D_{j}}=\delta_{E}$. To complete the proof, it suffices to show that $E \in$ $\operatorname{co}\left\{U \delta\left(U^{*}\right) \mid U \in \mathcal{U}(\mathcal{A})\right\}$. 
We denote by $S$ the collection of all (possibly empty) subsets of $\{1, \cdots, n\}$. There are $2^{n}$ sets in $S$. For $i \in\{1, \cdots, n\}$ and $K \in S$, we set $e_{K}^{i}=1$ if $i \in K$ and $e_{K}^{i}=-1$ if $i \notin K$. Let

$$
a_{i j}:=\sum_{K \in S} e_{K}^{i} e_{K}^{j}
$$

Clearly, $a_{i i}=\sum_{K} 1=2^{n}$.

Let $i \neq j$. We denote by $S_{1}$ the subset of $S$, such that every $K \in S_{1}$ satisfies $K \supset\{i, j\}$ and denote by $S_{2}$ the subset of $S$ such that every $K \in S_{2}$ satisfies that $K \cap\{i, j\}=\varnothing$. Clearly, there are $2^{n-2}$ sets in $S_{1}$ and $2^{n-2}$ sets in $S_{2}$. For every $K \in S_{1} \cup S_{2}$, we have $e_{K}^{i} e_{K}^{j}=1$. Note that there are $2^{n-1}$ sets in $S \backslash\left(S_{1} \cup S_{2}\right)$ and $e_{K}^{i} e_{K}^{j}=-1$ for every $K \in S \backslash\left(S_{1} \cup S_{2}\right)$. Hence, for $i \neq j$, we have

$$
a_{i j}=\sum_{K \in S} e_{K}^{i} e_{K}^{j}=\sum_{K \in S_{1} \cup S_{2}} e_{K}^{i} e_{K}^{j}+\sum_{K \in S \backslash\left(S_{1} \cup S_{2}\right)} e_{K}^{i} e_{K}^{j}=2^{n-1}-2^{n-1}=0 .
$$

For $\sigma \in S_{(n)}$, the set of all permutations of $\{1,2, \cdots, n\}$, and $K \in S$, we define a unitary operator

$$
U_{\sigma}^{K}:=\sum_{i=1}^{n} e_{K}^{i} B_{i, \sigma(i)}
$$

Then, by (6.4) and (6.5), we have

$$
\begin{aligned}
\sum_{\sigma \in S_{(n)}} \sum_{K \in S} U_{\sigma}^{K} \delta\left(\left(U_{\sigma}^{K}\right)^{*}\right) & =\sum_{\sigma \in S_{(n)}} \sum_{K \in S} \sum_{i, j} e_{K}^{i} e_{K}^{j} B_{j, \sigma(j)} \delta\left(B_{\sigma(i), i}\right) \\
& =\sum_{\sigma \in S_{(n)}} \sum_{i, j} B_{j, \sigma(j)} \delta\left(B_{\sigma(i), i}\right) \sum_{K \in S} e_{K}^{i} e_{K}^{j} \\
& =\sum_{\sigma \in S_{(n)}} \sum_{i} B_{i, \sigma(i)} \delta\left(B_{\sigma(i), i}\right) a_{i i} \\
& =2^{n} \sum_{i} \sum_{\sigma \in S_{(n)}} B_{i, \sigma(i)} \delta\left(B_{\sigma(i), i}\right) .
\end{aligned}
$$

For every $i, j$, there are $(n-1)$ ! permutations taking $i$ to $j$. Then, we obtain that

$$
\sum_{\sigma \in S_{(n)}} \sum_{K \in S} U_{\sigma}^{K} \delta\left(\left(U_{\sigma}^{K}\right)^{*}\right)=2^{n}(n-1) ! \sum_{i, j} B_{i j} \delta\left(B_{j i}\right)=2^{n} \frac{n !}{n} \sum_{i, j} B_{i j} \delta\left(B_{j i}\right) \stackrel{(6.3)}{=} 2^{n} n ! E,
$$

which implies that $E \in \operatorname{co}\left\{U \delta\left(U^{*}\right) \mid U \in \mathcal{U}(\mathcal{A})\right\}$.

The following theorem is the main result of this section, which is a semifinite version of the so-called Johnson-Parrott theorem [37] (see also [21, Chapter 10]). Another semifinite version of the Johnson-Parrott theorem (see [55]) shows that derivations from a type $I$ von Neumann subalgebra of $\mathcal{M}$ into $\mathcal{J}(\mathcal{M})$, the ideal of all compact operators in $\mathcal{M}$, are not necessarily inner. However, in the following theorem, we show that derivations from an arbitrary type $I$ von Neumann subalgebra of $\mathcal{M}$ into $\mathcal{C}_{0}(\mathcal{M}, \tau)$ are necessarily inner. 
Theorem 6.2. If $\mathcal{A}$ is a type I von Neumann subalgebra of $\mathcal{M}$, then for every derivation $\delta: \mathcal{A} \rightarrow \mathcal{C}_{0}(\mathcal{M}, \tau)$, there exists $K \in \mathcal{C}_{0}(\mathcal{M}, \tau) \cap K_{\delta}$ such that $\delta=\delta_{K}$.

Proof. Since $\mathcal{A}$ is a type $I$ von Neumann algebra, there exists a central partition of unity $\left\{Z_{n}: n \in \mathbb{N}\right\}$ such that $Z_{n} \mathcal{A}$ is of type $I_{n}$ and $Z_{0} \mathcal{A}$ is properly infinite. Recall that we may always assume that $\left.\delta\right|_{\mathcal{Z}(\mathcal{A})}=0$ (see Remark 4.4). We have $\delta\left(Z_{n} \mathcal{A}\right) \subset$ $Z_{n} \mathcal{C}_{0}(\mathcal{M}, \tau) Z_{n}$ for all $n \geq 0$. Since for $n \geq 1$, the algebra $Z_{n} \mathcal{A}$ is of type $I_{n}$, it follows from Proposition 6.1 that $\left.\delta\right|_{Z_{n} \mathcal{A}}=\delta_{K_{n}}$ for some $K_{n} \in Z_{n} \mathcal{C}_{0}(\mathcal{M}, \tau) Z_{n} \cap Z_{n} K_{\delta}$. In addition, by Theorem 5.3, there exists $K_{0} \in Z_{0} \mathcal{C}_{0}(\mathcal{M}, \tau) Z_{0} \cap Z_{0} K_{\delta}$ such that $\left.\delta\right|_{Z_{0} \mathcal{A}}=$ $\delta_{K_{0}}$. Set $K=\sum_{n=0}^{\infty} Z_{n} K_{n}$. Appealing to Theorem 3.9, we conclude that that $\delta=\delta_{K}$ and $K \in \mathcal{C}_{0}(\mathcal{M}, \tau) \cap K_{\delta}$.

\section{The Type $I I_{1}$ Case}

Recall that $\mathcal{M}$ is a semifinite von Neumann algebra with a faithful normal semifinite trace $\tau$. Let $\mathcal{A}$ be a von Neumann subalgebra of $\mathcal{M}$ and let $\delta: \mathcal{A} \rightarrow \mathcal{C}_{0}(\mathcal{M}, \tau)$ be a derivation. As we showed in Theorems 5.3 and 6.2, the derivation $\delta$ is inner provided that $\mathcal{A}$ is properly infinite or of type $I$. Hence, by Remark 3.4, to complete the proof of Theorem 1.2, it remains to consider the case when $\mathcal{A}$ is of type $I I_{1}$. We cover this remaining case in the present section.

The special case when $\mathcal{M}=B(\mathcal{H})$ (in this case, $\mathcal{C}_{0}(\mathcal{M}, \tau)$ is just $K(H)$ ) was first resolved by Popa (see [54, Theorem II]) by transforming the noncommutative framework of the problem into a commutative one. In [55], Popa and Rădulescu extended the results in $[37,54]$ to a semifinite case when $\delta$ takes values from $\mathcal{J}(\mathcal{M})$, the ideal of all compact operators in $\mathcal{M}$ (see also [32, Section 5] for the case when the smaller algebra is a type $I I_{1}$ factor, and [44] for the case when the smaller algebra is abelian or properly infinite which contains the center of $\mathcal{M}$ ).

In the setting of the present section, we consider a derivation $\delta: \mathcal{A} \rightarrow \mathcal{C}_{0}(\mathcal{M}, \tau)$, where $\mathcal{A}$ is a type $I I_{1}$ algebra. Since $\mathcal{C}_{0}(\mathcal{M}, \tau) \subset \mathcal{J}(\mathcal{M})$, the main result of [55] guarantees that there exists $T \in \mathcal{J}(\mathcal{M})$ such that $\delta=\delta_{T}$. Hence, to prove that $\delta$ : $\mathcal{A} \rightarrow \mathcal{C}_{0}(\mathcal{M}, \tau)$ is inner, it is sufficient to show that there exists $T^{\prime} \in \mathcal{A}^{\prime}$ such that $T-T^{\prime} \in \mathcal{C}_{0}(\mathcal{M}, \tau)$.

7.1. Some preliminaries. Let $\mathcal{M}_{\tau}:=\left\{X \in \mathcal{M} \mid \tau\left(X^{*} X\right)<\infty\right\}$ be the Hilbert-Schmidt class ideal in $\mathcal{M}$ equipped with the norm $\|X\|_{\tau}=\tau\left(X^{*} X\right)^{\frac{1}{2}}, X \in \mathcal{M}_{\tau}$. Let $\mathcal{H}_{\tau}$ be the Hilbert space completion of $\mathcal{M}_{\tau}$ in the norm $\|\cdot\|_{\tau}$, that is, $\mathcal{H}_{\tau}=L_{2}(\mathcal{M}, \tau) . \mathcal{M}$ is always regarded in its standard representation, acting on $\mathcal{H}_{\tau}$ by left multiplication.

In what follows, we introduce norms $\|\cdot\| \cdot \|$ and $\|\mid \cdot\|_{\text {ess }}$ on $\mathcal{M}$. The norms $\||\cdot|\|$ and \|\|$\cdot \|_{\text {ess }}$ play similar roles in this paper as the uniform and usual essential norms do in [37] and [54] (see also [21, Chapter 10]).

By the well-known Holmstedt formula (see e.g. [34, Theorem 4.1]), $\|\cdot\|_{L_{2}+L_{\infty}}$ defined by $\|f\|_{L_{2}+L_{\infty}}=\left(\int_{0}^{1} \mu(t ; f)^{2} d t\right)^{1 / 2}, f \in L_{2}(0, \infty)+L_{\infty}(0, \infty)$, is a complete norm on $L_{2}(0, \infty)+L_{\infty}(0, \infty)$. It follows immediately from the definition of the norm $\|\cdot\|_{L_{2}+L_{\infty}}$ that $\left(L_{2}+L_{\infty}\right)(0, \infty)$ equipped with the norm $\|\cdot\|_{L_{2}+L_{\infty}}$ is a strongly symmetric space. Hence, $\left(L_{2}+L_{\infty}\right)(\mathcal{M}, \tau)$ is a strongly symmetric operator space equipped with norm $\|\cdot\|_{L_{2}+L_{\infty}}$ defined by $\|T\|_{L_{2}+L_{\infty}}=\left(\int_{0}^{1} \mu(t ; T)^{2} d t\right)^{1 / 2}, T \in\left(L_{2}+L_{\infty}\right)(\mathcal{M}, \tau)$ (see e.g. $[28,30,45])$.

Note that the definition of $\||\cdot|||$ below is rather different from that of the uniform norms introduced in [37], [54] and [55, 2.3]. 
Definition 7.1. For every $T \in \mathcal{M}$, we define $\|T\|\|:=\mid\|_{L_{2}+L_{\infty}}$. It is clear that $\|T\| \mid \leq$ $\|T\|_{\infty}$.

Proposition 7.2. If $T_{1}, T_{2}, T \in \mathcal{M}$, then $\left\|T_{1} T T_{2}\right\| \leq\left\|T_{1}\right\|_{\infty}\|\mid\| T\|\| T_{2} \|_{\infty}$ and $\|T\| \|=$ $\left\|\left|T^{*}\right|\right\||=|\||T||\||$.

Lemma 7.3. Let $K \in \mathcal{C}_{0}(\mathcal{M}, \tau)$ and $\left\{E_{n}\right\}$ be a sequence of mutually orthogonal projections in $\mathcal{M}$. Then, we have $\left\|K E_{n}\right\| \mid \rightarrow_{n} 0$ and $\left\|E_{n} K\right\| \| \rightarrow_{n} 0$.

Proof. By Proposition 7.2, we have $\left\|\mid E_{k} K\right\|\|=\| K^{*} E_{k} \|$. Therefore, it is sufficient to show that $\left\|K E_{k}\right\| \mid \rightarrow_{k} 0$.

Since $K$ is a $\tau$-compact operator, the projection $E^{|K|}(\varepsilon, \infty)$ is $\tau$-finite for every $\varepsilon>0$. In particular, $K E^{|K|}(\varepsilon, \infty) \in \mathcal{F}(\mathcal{M}, \tau)$. Since $\left\|K-K E^{|K|}(\varepsilon, \infty)\right\| \leq\left(\int_{0}^{1} \varepsilon^{2} d t\right)^{1 / 2}=$ $\varepsilon$, it follows that $K \in \overline{\mathcal{F}(\mathcal{M}, \tau)}\|\cdot\| \|$. Then, [28, Proposition 56] together with [27, Theorem 6.13 (iii)] implies that $\|\left|K\left(\vee_{n \geq k} E_{n}\right)\right| \mid \rightarrow_{k} 0$. By Proposition 7.2, we have $\left\|K E_{k}\right\|\left|\leq\left\|K\left(\vee_{n \geq k} E_{n}\right) \mid\right\| \rightarrow_{k} 0\right.$.

Theorem 7.4. ||$|\cdot|||$ is inferior semicontinuous with respect to the weak operator topology, that is, if a net $\left\{T_{i}\right\}$ converges $T$ in the weak operator topology, then $\|T\| \leq \leq$ $\lim \sup _{i}\left\|T_{i}\right\|$.

Proof. By [47, Lemma 2.3.18], we may assume without loss of generality that $\mathcal{M}$ is atomless. We have that $\mu(T)^{2}=\mu(|T|)^{2}=\mu\left(|T|^{2}\right)$ (see [47, Corollary 2.3.17 (d)]). Therefore, by [47, Lemma 3.3.2], we have that

$$
\int_{0}^{1} \mu(s ; T)^{2} d s=\int_{0}^{1} \mu\left(s ;|T|^{2}\right) d s=\sup \left\{\|T P\|_{\tau}^{2}: P \in \mathcal{P}(\mathcal{M}), \tau(P) \leq 1\right\} .
$$

and, similarly,

$$
\int_{0}^{1} \mu\left(s ; T_{i}\right)^{2} d s=\int_{0}^{1} \mu\left(s ;\left|T_{i}\right|^{2}\right) d s=\sup \left\{\left\|T_{i} P\right\|_{\tau}^{2}: P \in \mathcal{P}(\mathcal{M}), \tau(P) \leq 1\right\} .
$$

Let $P \in \mathcal{P}(\mathcal{M})$ be such that $\tau(P) \leq 1$. Since $\left|\tau\left(P T^{*} T_{i} P\right)\right| \leq\left\|T_{i} P\right\|_{\tau}\|T P\|_{\tau}$, it follows that $\|T P\|_{\tau}^{2}=\tau\left(P T^{*} T P\right)=\lim _{i}\left|\tau\left(P T^{*} T_{i} P\right)\right| \leq \lim \sup _{i}\left\|T_{i} P\right\|_{\tau}\|T P\|_{\tau}$. Hence, we have

$$
\|T P\|_{\tau} \leq \limsup _{i}\left\|T_{i} P\right\|_{\tau} \stackrel{(7.2)}{\leq} \limsup _{i}\left\|T_{i}\right\|,
$$

which together with (7.1) implies that $\|T\| \leq \lim \sup _{i}\left\|\mid T_{i}\right\|$.

In [55, 2.6], an essential norm was defined in terms of the compact ideal in a semifinite von Neumann algebra. Below, we introduce the essential norm with respect to the $\tau$ compact ideal.

Definition 7.5. For $T \in \mathcal{M}$, we define $\|T\|_{\text {ess }}:=\inf \left\{\left\||T-K \|| \mid K \in \mathcal{C}_{0}(\mathcal{M}, \tau)\right\}\right.$.

The norm $\|\mid \cdot\|_{\text {ess }}$ can be described in terms of the singular value function.

Proposition 7.6. $\|T\|_{\text {ess }}=\mu(\infty ; T)$ for every $T \in \mathcal{M}$. 
Proof. We may assume that $\mu(\infty ; T)=\varepsilon$ for some $\varepsilon>0$. For any $K \in \mathcal{C}_{0}(\mathcal{M}, \tau)$ and $\Delta>0$, there exists $t_{0}>0$ such that $\mu(t ; T)-\mu(t ; K) \geq \varepsilon-\Delta$ for every $t>t_{0}$. Using [23, Theorem 3.4] (see also [28]), we obtain that

$$
\varepsilon-\Delta \prec \prec \mu(T)-\mu(K) \prec \prec \mu(T-K)
$$

for any $K \in \mathcal{C}_{0}(\mathcal{M}, \tau)$ and $\Delta>0$. By Lemma 2.6, the latter condition guarantees that $\mu(T-K) \geq \varepsilon-\Delta$. Thus, $\|T-K\|=\left(\int_{0}^{1} \mu(t ; T-K)^{2} d t\right)^{1 / 2} \geq \varepsilon-\Delta$.

Since $K$ and $\Delta$ are arbitrary, it follows that $\|T\|_{\text {ess }} \geq \varepsilon$.

To prove the converse inequality, assume that $\Delta>0$ and choose $t>0$ such that $\mu(t ; T) \leq \varepsilon+\Delta$. By Lemma $2.4, E^{|T|}(\varepsilon+\Delta, \infty)$ is $\tau$-finite. In particular, $T E^{|T|}(\varepsilon+$ $\Delta, \infty) \in \mathcal{C}_{0}(\mathcal{M}, \tau)$. It follows from the definition of $\|\cdot \cdot\|_{\text {ess }}$ that

$$
\begin{aligned}
\|T\|_{\text {ess }} & \leq\|\mid\| T-T E^{|T|}(\varepsilon+\Delta, \infty)\|\| \\
& =\|\mid\| E^{|T|}[0, \varepsilon+\Delta]\|\| \\
& =\left(\int_{0}^{1} \mu\left(t ; T E^{|T|}[0, \varepsilon+\Delta]\right)^{2} d t\right)^{1 / 2} \\
& \leq \varepsilon+\Delta .
\end{aligned}
$$

Since $\Delta$ is arbitrary, we obtain that $\|T\|_{\text {ess }}=\varepsilon$.

Let $T_{1}, T_{2} \in \mathcal{M}$ be two operators which are disjoint from the left and the right. The essential norm of $T_{1}+T_{2}$ with respect to $\mathcal{J}(\mathcal{M})$ (see [55, Definition 2.6]) does not necessarily equal the maximum of the essential norms of $T_{1}$ and $T_{2}$ with respect to $\mathcal{J}(\mathcal{M})$ (see [55, Section 2.7]). However, similar to the usual essential norm in $B(\mathcal{H})$ (see e.g. $[21,37])$, the essential norm with respect to $\mathcal{C}_{0}(\mathcal{M}, \tau)$ has the following property for disjointly supported operators.

Proposition 7.7. Let $T \in \mathcal{M}$ and let $P_{1}, P_{2}$ be mutually orthogonal projections in $\mathcal{M}$. We have

$$
\max _{i}\left\|P_{i} T P_{i}\right\|_{\text {ess }}=\|\| P_{1} T P_{1}+P_{2} T P_{2} \|_{\text {ess }} .
$$

Proof. Without loss of generality, we assume that both $P_{1} T P_{1}$ and $P_{2} T P_{2}$ are not $\tau$ compact with $\varepsilon_{1}:=\mu\left(\infty ; P_{1} T P_{1}\right) \geq \mu\left(\infty ; P_{2} T P_{2}\right)=$ : $\varepsilon_{2}$. By Lemma 2.4 , for every $\Delta>0$, we have

$$
\begin{aligned}
\tau\left(E^{\left|P_{1} T P_{1}+P_{2} T P_{2}\right|}\left(\varepsilon_{1}+\Delta, \infty\right)\right) & =\tau\left(E^{\left|P_{1} T P_{1}\right|}\left(\varepsilon_{1}+\Delta, \infty\right)\right)+\tau\left(E^{\left|P_{2} T P_{2}\right|}\left(\varepsilon_{1}+\Delta, \infty\right)\right) \\
& =M<\infty
\end{aligned}
$$

for some $M>0$. Hence, using again Lemma 2.4, we obtain that

$$
\mu\left(\infty ; P_{1} T P_{1}+P_{2} T P_{2}\right) \leq \mu\left(M ; P_{1} T P_{1}+P_{2} T P_{2}\right) \leq \varepsilon_{1}+\Delta .
$$

Moreover, since $\left|P_{1} T P_{1}\right| \leq\left|P_{1} T P_{1}\right|+\left|P_{2} T P_{2}\right|=\left|P_{1} T P_{1}+P_{2} T P_{2}\right|$, it follows that

$$
\varepsilon_{1}=\mu\left(\infty ; P_{1} T P_{1}\right) \leq \mu\left(\infty ; P_{1} T P_{1}+P_{2} T P_{2}\right) .
$$

Since $\Delta$ is arbitrary, we conclude that $\mu\left(\infty ; P_{1} T P_{1}+P_{2} T P_{2}\right)=\varepsilon_{1}$. The assertion now follows from Proposition 7.6. 
Remark 7.8. Note that for any semifinite von Neumann algebra $\mathcal{A}$ there exist pairwise orthogonal central projections $P_{i}$ with $\sum_{i} P_{i}=1$ such that each $\mathcal{Z}(\mathcal{A})_{P_{i}}$ is countably decomposable. Hence, combining Theorem 3.9 together with Theorem 4.2, we may assume, without loss of generality, that the center $\mathcal{Z}(\mathcal{A})$ of the von Neumann subalgebra $\mathcal{A}$ of $\mathcal{M}$ is countably decomposable. In particular, since $\mathcal{A}$ is of type $I I_{1}$, we can always assume that $\mathcal{A}$ is a countably decomposable type $I I_{1}$ von Neumann algebra (see e.g. [42, Corollary 8.2.9]).

7.2. Some continuity results. In this subsection, we study the continuity of derivations $\delta: \mathcal{A} \rightarrow \mathcal{C}_{0}(\mathcal{M}, \tau)$. Similar results with respect to the ideal $\mathcal{J}(\mathcal{M})$ can be found in $[55$, Section 4]. In this part, unless otherwise stated, we always assume that algebra $\mathcal{A}$ is a countably decomposable type $I I_{1}$ von Neumann subalgebra of $\mathcal{M}$ and therefore $\mathcal{A}$ has a normal faithful finite trace $\tau_{\mathcal{A}}$. For every $X \in \mathcal{A}$, we denote

$$
\|X\|_{2}:=\tau_{\mathcal{A}}\left(X^{*} X\right)^{1 / 2}
$$

Proposition 7.9. Let $\delta: \mathcal{A} \rightarrow \mathcal{C}_{0}(\mathcal{M}, \tau)$ be a derivation. Then, $\delta$ is continuous from the unit ball of $\mathcal{A}$ with the strong operator topology into $\mathcal{C}_{0}(\mathcal{M}, \tau)$ equipped with the norm $\| \cdot|| \mid$.

Proof. By Ringrose's theorem [56], the mapping $\delta:\left(\mathcal{A},\|\cdot\|_{\infty}\right) \rightarrow\left(\mathcal{C}_{0}(\mathcal{M}, \tau),\|\cdot\| \|\right)$ is continuous. Hence, denoting by $\|\delta\|$ the operator norm this mapping, we can assume that $\|\delta\| \leq 1$.

We firstly prove that if $\left\{P_{n}\right\}_{n \in \mathbb{N}}$ is a sequence of projections in $\mathcal{A}$ with $\tau_{\mathcal{A}}\left(P_{n}\right) \rightarrow$ 0 , then $\left\|\delta\left(P_{n}\right)\right\| \| \rightarrow 0$. Suppose that $\left\|\delta\left(P_{n}\right)\right\| \|$ does not converge to 0 . Passing to a subsequence, if necessary, we may assume that $\left\|\delta\left(P_{n}\right)\right\| \| \geq c$ for some $c>0$ for all $n$ and that $\sum \tau_{\mathcal{A}}\left(P_{n}\right)<\infty$. Define $G_{n}:=\vee_{k \geq n} P_{k}$. We have

$$
\tau_{\mathcal{A}}\left(G_{n}\right) \leq \sum_{k \geq n} \tau_{\mathcal{A}}\left(P_{k}\right) \rightarrow 0
$$

as $n \rightarrow \infty$. Denote by $S_{n, m}$ the support of $P_{m} G_{n} P_{m}$. It is clear that $S_{n, m} \leq P_{m}$. Moreover, since $S_{n, m}=l\left(P_{m} G_{n} P_{m}\right)=r\left(P_{m} G_{n} P_{m}\right)$, it follows that $l\left(P_{m} G_{n} P_{m}\right) \leq$ $l\left(P_{m} G_{n}\right) \sim r\left(P_{m} G_{n}\right) \leq G_{n}$, i.e. $S_{n, m} \preceq G_{n}$. Therefore, $\tau_{\mathcal{A}}\left(S_{n, m}\right) \leq \tau_{\mathcal{A}}\left(G_{n}\right) \rightarrow_{n} 0$ for each $m$. Since $\left\{G_{n}\right\}_{n}$ is decreasing, it follows that for every fixed $m$, the sequence $\left\{P_{m} G_{n} P_{m}\right\}_{n}$ is decreasing, and so, $\left\{S_{n, m}\right\}$ is decreasing, too. In particular, $S_{n, m} \downarrow_{0}$ as $n \rightarrow \infty$. Thus, $\left\{P_{m}-S_{n, m}\right\}_{n}$ increases to $P_{m}$. Since $\delta$ is continuous in weak* operator topology (see [37, Lemma 1.3]), we obtain that $\left\{\delta\left(P_{m}-S_{n, m}\right)\right\}$ is convergent to $\delta\left(P_{m}\right)$ in the weak* operator topology. By the inferior semicontinuity of the norm $\|\mid \cdot\|$ (see Theorem 7.4), it follows that for a fixed $m$, we can find a sufficiently large $n$ such that

$$
\left\|\delta\left(P_{m}-S_{n, m}\right)\right\| \geq \geq \frac{c}{2} .
$$

Thus, by induction, we can find an increasing sequence of integers $n_{1}, n_{2}, \cdots$ such that for every $k$, the projection $H_{k}:=P_{n_{k}}-S_{n_{k+1}, n_{k}}$ satisfies $\left\|\delta\left(H_{k}\right)\right\| \| \frac{c}{2}$. These projections also satisfy $\tau_{\mathcal{A}}\left(H_{k}\right) \leq \tau_{\mathcal{A}}\left(P_{n_{k}}\right) \rightarrow_{k} 0$. Moreover, since $H_{k} \leq P_{n_{k}}$ and $S_{n_{k+1}, n_{k}}$ is the support of $P_{n_{k}} G_{n_{k+1}} P_{n_{k}}$, by the definition of $H_{k}$ we get

$$
H_{k} G_{n_{k+1}} H_{k}=H_{k} P_{n_{k}} G_{n_{k+1}} P_{n_{k}} H_{k}=H_{k} P_{n_{k}} G_{n_{k+1}} P_{n_{k}} S_{n_{k+1}, n_{k}} H_{k}=0,
$$


which implies that $H_{k} G_{n_{k+1}}=0$. Recalling that $G_{n}=\vee_{k \geq n} P_{k}$, we conclude that $H_{k} H_{l}=0$ for every $l \geq k+1$, which means that $H_{k}$ are mutually orthogonal projections.

Denote by $\mathcal{B}$ the abelian von Neumann subalgebra of $\mathcal{A}$ generated by $\left\{H_{k}\right\}$. By considering $\delta$ as a derivation from $\mathcal{B}$ into $\mathcal{C}_{0}(\mathcal{M}, \tau)$, we can apply Theorem 4.2 to obtain the existence of $K \in \mathcal{C}_{0}(\mathcal{M}, \tau)$, such that $\delta\left(H_{k}\right)=\delta_{K}\left(H_{k}\right)$ for $k \in \mathbb{N}$. On one hand, $\left\|\mid \delta\left(H_{k}\right)\right\| \| \geq \frac{c}{2}$. On the other hand, since $H_{n}$ are mutually orthogonal projections, Lemma 7.3 implies that $\left\|\delta\left(H_{k}\right)\right\|=\|\| \delta_{K}\left(H_{k}\right)\|\leq\|\left\|K H_{k}\right\| \mid+\left\|H_{k} K\right\| \| \rightarrow$, which is a contradiction.

Now, we turn to the general case. It is well-known that $\|\cdot\|_{2}$ induces the strong operator topology on the unit ball of $\mathcal{A}$. Hence, it suffices to prove that if $\left\{X_{n}\right\}_{n}$ is a bounded sequence in $\mathcal{A}$ with $\left\|X_{n}\right\|_{2} \rightarrow_{n} 0$, then $\left\|\mid \delta\left(X_{n}\right)\right\| \| \rightarrow_{n} 0$. Without loss of generality, we may assume that every element in $\left\{X_{n}\right\}$ is positive and $\left\|X_{n}\right\|_{\infty} \leq 1$ (see e.g. a similar argument in the penultimate paragraph of the proof of [55, Proposition 4.1]).

Let $0 \leq X \leq \mathbf{1}$ be arbitrary. Let $A_{m}=\cup_{i=1}^{2^{m-1}}\left((2 i-1) / 2^{m}, 2 i / 2^{m}\right], m \geq 1$. We define

$$
k_{m}:=\chi_{A_{m}}, m \geq 1 .
$$

Note that for every $\lambda \in[0,1]$, we have $\lambda=\sum_{m \geq 1} 2^{-m} k_{m}(\lambda)$. By functional calculus, we have

$$
\begin{aligned}
X & =\int \lambda d E_{\lambda}^{X}=\int \sum_{m \geq 1} 2^{-m} k_{m}(\lambda) d E_{\lambda}^{X} \\
& =\sum_{m \geq 1} 2^{-m} \int k_{m}(\lambda) d E_{\lambda}^{X}=\sum_{m \geq 1} 2^{-m} E_{A_{m}}^{X} .
\end{aligned}
$$

Thus, for every $X_{n}$, we can write the dyadic decomposition

$$
X_{n}:=\sum_{m \geq 1} 2^{-m} e_{m}^{n},
$$

where $e_{m}^{n}:=E_{A_{m}}^{X_{n}}$.

Since $\left\|X_{n}\right\|_{2} \rightarrow_{n} 0$, it follows that $\tau_{\mathcal{A}}\left(e_{m}^{n}\right) \rightarrow_{n} 0$ for each $m \geq 1$. Let $\varepsilon>0$ be fixed and choose $m_{0} \geq 1$ such that $2^{-m_{0}} \leq \frac{\varepsilon}{2}$. By the first part of the proof, there exists $n_{0}$ such that for every $n \geq n_{0},\left\|\mid \delta\left(e_{m}^{n}\right)\right\| \|<\frac{\varepsilon}{2}$ for any $m \leq m_{0}$. Recall that $\|\delta\| \leq 1$. For $n \geq n_{0}$, we infer that

$$
\begin{aligned}
\left\|\delta\left(X_{n}\right)\right\| & \leq \sum_{m=1}^{m_{0}} 2^{-m}\left\|\delta\left(e_{m}^{n}\right)\right\|+\|\delta\| \cdot\left\|\sum_{m>m_{0}} 2^{-m} e_{m}^{n}\right\|_{\infty} \\
& \leq \sum_{m=1}^{m_{0}} 2^{-m}\left\|\delta\left(e_{m}^{n}\right)\right\|+\|\delta\| \cdot \sum_{m>m_{0}} 2^{-m} \\
& \leq\left(\sum_{m=1}^{m_{0}} 2^{-m}\right) \frac{\varepsilon}{2}+1 \cdot \frac{\varepsilon}{2} \cdot \sum_{m=1}^{\infty} 2^{-m} \\
& \leq \varepsilon
\end{aligned}
$$

which completes the proof. 
Recall that $K_{\delta}=\overline{c o}^{w o}\left\{U \delta\left(U^{*}\right) \mid U \in \mathcal{U}(\mathcal{A})\right\}$. Proposition 7.9 immediately implies the following corollary.

Corollary 7.10. Given $\beta>0$, there exists $\alpha>0$ such that

$$
\|T X\| \leq \beta \text { and }\|X T\| \mid \leq \beta
$$

for all $T \in K_{\delta}$ and $X \in \mathcal{A},\|X\|_{\infty} \leq 1,\|X\|_{2} \leq \alpha$.

Proof. By Proposition 7.9, there exists $\alpha>0$ such that $\|\delta(X)\|<\beta / 3$ for every $X \in \mathcal{A}$ with $\|X\|_{\infty} \leq 1$ and $\|X\|_{2}<\alpha$. For a unitary element $U$ in $\mathcal{A}$, we have $U \delta\left(U^{*}\right) X=U \delta\left(U^{*} X\right)-\delta(X)$ and $\left\|U^{*} X\right\|_{2}=\|X\|_{2}$, which implies that

$$
\left\|U \delta\left(U^{*}\right) X\left|\|\leq\| U \delta\left(U^{*} X\right)\|\|+\|\delta(X)\|\right|<\frac{2}{3} \beta .\right.
$$

By taking convex combinations of $U \delta\left(U^{*}\right)$ and using the inferior semi-continuity of norm $\|\cdot\| \mid \|$ in the weak operator topology (see Theorem 7.4), we get $\|T X\| \mid \leq \beta$ for all $T \in K_{\delta}$. The symmetricity of the norm $\|\cdot\| \|$ (see Proposition 7.2) implies that $\||X T \|| \leq \beta$.

The following proposition is the main result of the present subsection, which is the key in the proof of Theorem 7.13 below.

Proposition 7.11. Let $\delta: \mathcal{A} \rightarrow \mathcal{C}_{0}(\mathcal{M}, \tau)$ be a derivation. If $T \in K_{\delta}$ is such that $\delta=\delta_{T}$ on $\mathcal{A}$, then $T \in \mathcal{C}_{0}(\mathcal{M}, \tau)$.

Proof. Since $\mathcal{A}$ is of type $I I_{1}$, there exists a decreasing sequence of projections $\left\{E_{n}\right\}_{n \geq 0}$ in $\mathcal{A}$ with $E_{0}=\mathbf{1}, E_{n+1} \sim E_{n}-E_{n+1}$ for all $n \geq 0$ (see e.g. [42, Lemma 6.5.6]).

Using mathematical induction, we show that $\left\|E_{n} T E_{n}\right\|\left\|_{\text {ess }}=\right\| T \|_{\text {ess }}$ for all $n$. For $n=0$, the assertion is trivial. Assume that $\left\|E_{k} T E_{k}\right\|\left\|_{\text {ess }}=\right\| T \|_{\text {ess }}$ for all $k \leq n$ for some fixed $n \geq 0$. For every $n$, by [64, Chapter XIV, Lemma 2.1], there is a unitary element $U_{n} \in \mathcal{A}$ such that

$$
U_{n}^{*} E_{n+1} U_{n}=E_{n}-E_{n+1} .
$$

Since $\delta=\delta_{T}$, it follows that that $U_{n}^{*} T U_{n}-T=\delta\left(U^{*}\right) U \in \mathcal{C}_{0}(\mathcal{M}, \tau)$. Therefore, by Proposition 7.6 and Definition 7.5, we have

$$
\begin{aligned}
\left\|E_{n+1} T E_{n+1}\right\|_{\text {ess }} & =\left\|U_{n} E_{n+1} T E_{n+1} U_{n}^{*}\right\| \|_{\text {ess }} \\
& =\left\|U_{n} E_{n+1} U_{n}^{*} T U_{n} E_{n+1} U_{n}^{*}\right\|_{\text {ess }} \\
& \stackrel{(7.4)}{=}\left\|\left(E_{n}-E_{n+1}\right) T\left(E_{n}-E_{n+1}\right)\right\|_{\text {ess }} .
\end{aligned}
$$

Now, using now Proposition 7.7, we infer that

$$
\begin{aligned}
& \quad\left\|E_{n} T E_{n}\right\| \|_{e s s} \\
& =\| \mid E_{n+1} T E_{n+1}+\left(E_{n}-E_{n+1}\right) T\left(E_{n}-E_{n+1}\right) \\
& \quad+\delta\left(E_{n+1}\right)\left(E_{n}-E_{n+1}\right)+\delta\left(E_{n}-E_{n+1}\right) E_{n+1} \|_{\text {ess }} \\
& =\left\|E_{n+1} T E_{n+1}+\left(E_{n}-E_{n+1}\right) T\left(E_{n}-E_{n+1}\right)\right\| \|_{\text {ess }} \\
& \stackrel{(7.3)}{=} \max \left\{\left\|E_{n+1} T E_{n+1}\right\|_{\text {ess }},\|\|\left(E_{n}-E_{n+1}\right) T\left(E_{n}-E_{n+1}\right)\|\|_{e s s}\right\} \\
& \stackrel{(7.5)}{=}\left\|E_{n+1} T E_{n+1}\right\| \|_{\text {ess }} .
\end{aligned}
$$


Therefore, $\|\mid T\|=\left\|E_{n} T E_{n}\right\|_{\text {ess }}=\left\|E_{n+1} T E_{n+1}\right\|_{e s s}$, which concludes the induction argument.

Assume now that $T \notin \mathcal{C}_{0}(\mathcal{M}, \tau)$, that is,

$$
\left\|E_{n} T E_{n}\right\|_{\text {ess }}=\|T\|_{\text {ess }}=: c>0
$$

for all $n$.

Since $\vee_{k \geq n} E_{k} \downarrow_{n} 0$, we have $\tau_{\mathcal{A}}\left(E_{n}\right) \leq \tau_{\mathcal{A}}\left(\vee_{k \geq n} E_{k}\right) \rightarrow_{n} 0$. Since $T \in K_{\delta}$ and $\left\|E_{n}\right\|_{2} \rightarrow 0$, Proposition 7.2 and Corollary 7.10 imply that

$$
\left\|E_{n} T E_{n}\right\|\left|\leq\left\|T E_{n}\right\|\right| \rightarrow_{n} 0,
$$

which is a contradiction to (7.6). Thus, $T \in \mathcal{C}_{0}(\mathcal{M}, \tau)$, as required.

7.3. The proof for the main result: the type $I I_{1}$ case. Before proceeding to the proof of Theorem 7.13, we prove the special case when $\mathcal{Z}(\mathcal{M})$ is of countable type by using the auxiliary results obtained in Sect. 7.2 and [55, Section 7.4]. To prove the case for $\mathcal{J}(\mathcal{M})$, several reductions are needed in [55, Section 7]. However, rather than repeating the proof in [55, Section 7], we use the main result of [55] in the proof of the following proposition, which makes our proof more efficient.

Proposition 7.12. If the center $\mathcal{Z}(\mathcal{M})$ of $\mathcal{M}$ is countably decomposable, then every derivation $\delta$ from a type $I_{1}$ von Neumann subalgebra $\mathcal{A}$ of $\mathcal{M}$ into $\mathcal{C}_{0}(\mathcal{M}, \tau)$ is inner. Moreover, the element $T \in \mathcal{C}_{0}(\mathcal{M}, \tau)$ implementing $\delta$ lies in $K_{\delta}$.

Proof. It is proved in [55] that every derivation from a type $I I_{1}$ von Neumann subalgebra $\mathcal{A}$ into $\mathcal{J}(\mathcal{M})$ is implemented by some element in $\mathcal{J}(\mathcal{M})$. Noticing that $\mathcal{C}_{0}(\mathcal{M}, \tau) \subset$ $\mathcal{J}(\mathcal{M})$, we conclude that there exists an element $K \in \mathcal{J}(\mathcal{M})$ such that $\delta=\delta_{K}$. Since $\mathcal{Z}(\mathcal{M})$ is countably decomposable, by [55, Lemma 4.6] (note that this Lemma requires the condition that $\mathcal{Z}(\mathcal{M})$ is countably decomposable), there is a $\bar{T} \in \overline{c o}^{\text {wo }}\left\{\delta(U) U^{*} \mid\right.$ $U \in \mathcal{U}(\mathcal{A})\}=-K_{\delta}$ such that $\delta(\cdot)=[\bar{T}, \cdot]=\delta_{-\bar{T}}(\cdot)$. Now, let $T=-\bar{T}$. Then, $T \in K_{\delta}$ with $\delta=\delta_{T}$. It follows from Proposition 7.11 that $T \in \mathcal{C}_{0}(\mathcal{M}, \tau)$.

In the following theorem, we remove the condition that $\mathcal{Z}(\mathcal{M})$ is countably decomposable imposed in Proposition 7.12, proving the main result of this section.

Theorem 7.13. Every derivation $\delta$ from a type $I_{1}$ von Neumann subalgebra $\mathcal{A}$ of $\mathcal{M}$ into $\mathcal{C}_{0}(\mathcal{M}, \tau)$ is inner. Moreover, the element implementing $\delta$ lies in $K_{\delta}$.

Proof. Let $\left\{Z_{i} \in \mathcal{Z}(\mathcal{M})\right\}$ be a net of projections increasing to 1 such that $\mathcal{Z}\left(\mathcal{M}_{Z_{i}}\right)=$ $\mathcal{Z}(\mathcal{M})_{Z_{i}}$ is countably decomposable. Since $\mathcal{A}$ is assumed to be countably decomposable (see Remark 7.8), it follows that $\mathcal{A}_{Z_{i}}$ is also countably decomposable. Define $\delta_{i}: \mathcal{A}_{Z_{i}} \rightarrow$ $Z_{i} \mathcal{C}_{0}(\mathcal{M}, \tau) Z_{i}=\mathcal{C}_{0}\left(\mathcal{M}_{Z_{i}}, \tau\right)$ by $\delta_{i}\left(X Z_{i}\right)=Z_{i} \delta(X) Z_{i}$ for every $X \in \mathcal{A}$. Since $Z_{i} \in$ $\mathcal{Z}(\mathcal{M})$, it follows from Lemma 3.2 that $\delta_{i}$ are well-defined derivations.

By Proposition 7.12, there exists $K_{i} \in \mathcal{C}_{0}\left(\mathcal{M}_{Z_{i}}, \tau\right)$ with $K_{i} \in K_{\delta_{i}}$ such that $\delta_{i}=\delta_{K_{i}}$ on $\mathcal{A}_{Z_{i}}$. Since $\mathcal{U}\left(\mathcal{A}_{Z_{i}}\right)=\mathcal{U}(\mathcal{A}) Z_{i}$ (see e.g. [41, Proposition 5.5.5]), it follows that

$$
\begin{aligned}
K_{i} \in K_{\delta_{i}} & =\overline{c o}^{w o}\left\{U \delta_{i}\left(U^{*}\right) \mid U \in \mathcal{U}\left(\mathcal{A}_{Z_{i}}\right)\right\}=\overline{c o}^{w o}\left\{U Z_{i} \delta_{i}\left(U^{*} Z_{i}\right) \mid U \in \mathcal{U}(\mathcal{A})\right\} \\
& =\overline{c o}^{w o}\left\{U Z_{i} \delta\left(U^{*}\right) Z_{i} \mid U \in \mathcal{U}(\mathcal{A})\right\}=K_{\delta} Z_{i} .
\end{aligned}
$$

Hence, for every $i$, there exists $T_{i} \in K_{\delta}$ such that $K_{i}=Z_{i} T_{i} Z_{i}$. 
Note that $K_{\delta}$ is compact in the weak operator topology (see [20, Chapter IX, Proposition 5.5]). Let $T \in K_{\delta}$ be a limit point of a subnet of $\left\{T_{i}\right\}_{i}$ in the weak operator topology. Without loss of generality, we assume that $T_{i} \rightarrow{ }_{\text {wo }} T$. For every $X \in \mathcal{A}$, we have that

$$
Z_{i} \delta(X) Z_{i}=\delta_{K_{i}}(X)=\delta_{Z_{i} T_{i} Z_{i}}(X) .
$$

On one hand, since $Z_{i} \uparrow \mathbf{1}$, it follows that $Z_{i} \delta(X) Z_{i}=Z_{i} \delta(X) \rightarrow_{\text {so }} \delta(X)$ and therefore a fortiori $Z_{i} \delta(X) Z_{i} \rightarrow_{w o} \delta(X)$. On the other hand, since $\left\|U \delta\left(U^{*}\right)\right\|_{\infty} \leq$ $\|\delta\|_{\left(\mathcal{A},\|\cdot\|_{\infty}\right) \rightarrow\left(\mathcal{C}_{0}(\mathcal{M}, \tau),\|\cdot\|_{\infty}\right)}<\infty$ (see [56, Theorem 2]) and $T_{i} \in K_{\delta}$, it follows that $\left\|T_{i}\right\|_{\infty} \leq\|\delta\|_{\left(\mathcal{A},\|\cdot\|_{\infty}\right) \rightarrow\left(\mathcal{C}_{0}(\mathcal{M}, \tau),\|\cdot\|_{\infty}\right)}<\infty$ for every $i$. Hence, $Z_{i} T_{i} Z_{i} \rightarrow{ }_{w o} T$. Combining these two convergences, we conclude that

$$
\delta(X)=w o-\lim _{i} Z_{i} \delta(X) Z_{i}=w o-\lim _{i} \delta_{Z_{i} T_{i} Z_{i}}(X)=\delta_{T}(X),
$$

that is, $\delta=\delta_{T}$ on $\mathcal{A}$. Since $T \in K_{\delta}$, it follows from Proposition 7.11 that $T \in \mathcal{C}_{0}(\mathcal{M}, \tau)$.

\section{Conclusions and Applications}

The following theorem is the main result of the present paper, which shows that for an arbitrary von Neumann subalgebra $\mathcal{A}$ of $\mathcal{M}$, every derivation $\delta: \mathcal{A} \rightarrow \mathcal{C}_{0}(\mathcal{M}, \tau)$ is necessarily inner. We note that this result is in contrast to [55, Theorem 1.2] (see also [59, Example 4.1.8] and [10] for derivations acting on $C^{*}$-subalgebras). We do not impose any additional assumptions of the type $I$ summand of the algebra $\mathcal{A}$.

Theorem 8.1. Every derivation $\delta$ from a von Neumann subalgebra $\mathcal{A}$ of $\mathcal{M}$ into the ideal $\mathcal{C}_{0}(\mathcal{M}, \tau)$ of all $\tau$-compact operators is inner.

Proof. By Theorem 4.2, there exists $T \in \mathcal{C}_{0}(\mathcal{M}, \tau)$ such that $\left.\delta\right|_{\mathcal{Z}(\mathcal{A})}=\delta_{T}$. Replacing $\delta$ with $\delta-\delta_{T}$, we can assume that $\delta$ vanishes on $\mathcal{Z}(\mathcal{A})$. By Remark 3.4, it suffices to prove the assertion in the case when $\mathcal{A}$ is of type $I$, type $I I_{1}$ and properly infinite, separately. Hence, appealing to Theorem 6.2, Theorem 7.13 and Theorem 5.3, we conclude the proof.

In the particular case when $\mathcal{M}=B(\mathcal{H})$ and $\tau$ is the standard trace, our result recovers the results proved by Johnson and Parrott [37], and by Popa [54]. Furthermore, in the case of an arbitrary von Neumann algebra $\mathcal{M}$ equipped with a faithful normal finite trace $\tau$, we have that $\mathcal{C}_{0}(\mathcal{M}, \tau)=\mathcal{M}$ (see e.g. [47, Page 64]), and therefore, Theorem 8.1 guarantees that any derivation $\delta: \mathcal{A} \rightarrow \mathcal{M}$ is inner if $\mathcal{M}$ is equipped with a faithful normal finite trace. In the following corollary, we extend this result to a general finite von Neumann algebra $\mathcal{M}$, recovering the main result of [15, Section 5] using completely different approach.

Corollary 8.2. Every derivation $\delta$ from a von Neumann subalgebra $\mathcal{A}$ of a finite von Neumann algebra $\mathcal{M}$ into $\mathcal{M}$ is inner. Moreover, the element $K \in \mathcal{M}$ implementing $\delta$ can be chosen from $K_{\delta}$.

Proof. Since $\mathcal{M}$ is finite, it follows that there is a net $\left\{P_{i}\right\}$ of projections in $\mathcal{Z}(\mathcal{M})$ with $P_{i} \uparrow 1$ such that $\mathcal{M}_{P_{i}}$ is countably decomposable (see e.g. [42, Corollary 8.2.9] and [51, Theorem 1.3.6]). Hence, $\mathcal{M}_{P_{i}}$ has a faithful normal finite trace $\tau_{i}$ (see e.g. [51, Theorem 1.3.6]), that is, $\mathcal{M}_{P_{i}}=\mathcal{C}_{0}\left(\mathcal{M}_{P_{i}}, \tau_{i}\right)$. Therefore, by Theorem 8.1, the derivation 
$\delta_{i}: \mathcal{A}_{P_{i}} \rightarrow \mathcal{M}_{P_{i}}$ defined by $\delta_{i}\left(X P_{i}\right)=\delta(X) P_{i}$ is inner, that is, there exists $T_{i} \in \mathcal{M}_{P_{i}}$ such that $\delta_{i}=\delta_{T_{i}}$ on $\mathcal{A}_{P_{i}}$. By Proposition 3.10, there exists

$$
\begin{aligned}
K_{i} \in K_{\delta_{i}} & =\overline{c o}^{w o}\left\{U \delta_{i}\left(U^{*}\right) \mid U \in \mathcal{U}\left(\mathcal{A}_{P_{i}}\right)\right\}=\overline{c o}^{w o}\left\{U P_{i} \delta_{i}\left(U^{*} P_{i}\right) \mid U \in \mathcal{U}(\mathcal{A})\right\} \\
& =\overline{c o}^{w o}\left\{U \delta(U) P_{i} \mid U \in \mathcal{U}(\mathcal{A})\right\}=K_{\delta} P_{i}
\end{aligned}
$$

such that $\delta_{i}=\delta_{K_{i}}$ on $\mathcal{A}_{P_{i}}$ and there is a $K_{i}^{\prime} \in K_{\delta}$ such that $K_{i}=K_{i}^{\prime} P_{i}$. Since $K_{\delta}$ is compact in the weak operator topology (see [20, Chapter IX, Proposition 5.5]), there is a limit point $K \in K_{\delta}$ of a subnet of $\left\{K_{i}^{\prime}\right\}$ in the weak operator topology. Without loss of generality, we denote that $K_{i}^{\prime} \rightarrow{ }_{\text {wo }} K$. We have

$$
\delta(X) P_{i}=\delta_{i}(X)=\delta_{K_{i}}(X)=\delta_{K_{i}^{\prime} P_{i}}(X)
$$

for every $X \in \mathcal{A}$. Since $\left\{P_{i}\right\}$ converges strongly to the identity, it follows that $\delta=\delta_{K}$ on $\mathcal{A}$.

We conclude our paper with applications of Theorem 8.1 to derivations with values in a class of ideals of $\mathcal{M}$. We characterize a class of ideals $\mathcal{E}$ of $\mathcal{M}$ such that derivations with values in these ideals are automatically inner. Derivations with values in ideals of von Neumann algebras has been widely studied in the last decades (see e.g. [6,10-14,19, $32,33,37,44,54,55]$, although this list is far from a comprehensive list of references). For completeness, we recall some of the established results for derivations with values in ideals.

For an arbitrary von Neumann algebra $\mathcal{M}$ and any (not necessarily closed) ideal $\mathcal{E}$ of $\mathcal{M}$, it is known $[11,12]$ that any derivation $\delta: \mathcal{M} \rightarrow \mathcal{E}$ is inner. However, when one considers derivations $\delta: \mathcal{A} \rightarrow \mathcal{E}$, where $\mathcal{A}$ is a von Neumann subalgebra of $\mathcal{M}$, there are examples of non-inner derivations [55, Theorem 1.2]. For a semifinite von Neumann algebra $\mathcal{M}$ equipped with a faithful normal semifinite trace $\tau$, and a $C^{*}$-subalgebra $\mathcal{A}$ of $\mathcal{M}$, it is proved in [10] that a derivation $\delta$ defined on $\mathcal{A}$ is necessarily inner provided that the values $\delta$ belong to the ideal $E(\mathcal{M}, \tau) \cap \mathcal{M}$ generated by a fully symmetric function space $E(0, \infty)$ having the Fatou property and order continuous norm (see e.g. Sect. 2 and [28] for precise definitions). In Theorem 8.3 below, we show that the same result holds for derivations $\delta: \mathcal{A} \rightarrow E(\mathcal{M}, \tau) \cap \mathcal{M}$ with significantly weaker conditions on the symmetric space $E(\mathcal{M}, \tau)$ provided that $\mathcal{A}$ is a von Neumann subalgebra of $\mathcal{M}$. The conditions we impose on $E(\mathcal{M}, \tau)$ is that $E(\mathcal{M}, \tau) \subset S_{0}(\mathcal{M}, \tau)$ is a strongly symmetric space having the Fatou property. Thus, we significantly extend [44, Theorem 14].

We note that the Fatou property is an analogue of the so-called "dual normal" property of bimodules over von Neumann algebras (see e.g. [21] and [61]). It is known that every derivation from a hyperfinite von Neumann algebra $\mathcal{A}$ into a dual normal $\mathcal{A}$-bimodule is inner (see e.g. [56, Theorem 2] and [61, Theorem 2.4.3]). However, no additional conditions on the von Neumann subalgebra are needed in our setting.

Theorem 8.3. Let $\mathcal{M}$ be a semifinite von Neumann algebra equipped with a faithful normal semifinite trace $\tau$, let $E(\mathcal{M}, \tau)$ be a strongly symmetric space with the Fatou property and let $\mathcal{A}$ be a von Neumann subalgebra of $\mathcal{M}$. Then every derivation $\delta$ from $\mathcal{A}$ into $E(\mathcal{M}, \tau) \cap \mathcal{C}_{0}(\mathcal{M}, \tau)$ is necessarily inner, that is, there exists $T \in E(\mathcal{M}, \tau) \cap$ $\mathcal{C}_{0}(\mathcal{M}, \tau)$ such that $\delta=\delta_{T}$.

Proof. For any symmetric space $E(\mathcal{M}, \tau)$ such that $E(\mathcal{M}, \tau) \nsubseteq S_{0}(\mathcal{M}, \tau)$, there exists an element $X \in E(\mathcal{M}, \tau)$ such that $\mu(X) \geq \alpha \chi_{(0, \infty)} \geq \alpha \mu(\mathbf{1})$ for some $\alpha>0$ (see Sect. 2.3 and Definition 2.1). By Definition 2.5, we obtain that $1 \in E(\mathcal{M}, \tau)$, which implies that $\mathcal{C}_{0}(\mathcal{M}, \tau) \subset \mathcal{M} \subset E(\mathcal{M}, \tau)$ (see Sect. 2.2). That is, $E(\mathcal{M}, \tau) \cap \mathcal{C}_{0}(\mathcal{M}, \tau)=$ 
$\mathcal{C}_{0}(\mathcal{M}, \tau)$. By Theorem 8.1, it is sufficient to prove the case when $E(\mathcal{M}, \tau) \subset S_{0}(\mathcal{M}, \tau)$. In particular, $E(\mathcal{M}, \tau) \cap \mathcal{C}_{0}(\mathcal{M}, \tau)=E(\mathcal{M}, \tau) \cap \mathcal{M}$.

We first assume that the carrier projection of $E(\mathcal{M}, \tau)$ is $\mathbf{1}$. By [28, Theorem 32], we have that $E(\mathcal{M}, \tau)=E(\mathcal{M}, \tau)^{\times \times}$, that is, $E(\mathcal{M}, \tau)$ is the Köthe dual of $E(\mathcal{M}, \tau)^{\times}$. Since $E(\mathcal{M}, \tau) \cap \mathcal{M} \subset \mathcal{C}_{0}(\mathcal{M}, \tau)$, it follows from Theorem 8.1 and Proposition 3.10 that there is a $T \in K_{\delta}$ such that $\delta=\delta_{T}$. Hence, there exists a net $\left\{T_{i}\right\} \subset \operatorname{co}\left\{U \delta\left(U^{*}\right) \mid\right.$ $U \in \mathcal{U}(\mathcal{A})\} \subset E(\mathcal{M}, \tau) \cap \mathcal{C}_{0}(\mathcal{M}, \tau)$ such that $T_{i} \rightarrow_{\text {so }} T$ with

$$
\sup _{i} \tau\left(\left|T_{i} X\right|\right) \leq \sup _{i}\left\|T_{i}\right\|_{E} \leq\|\delta\|_{\mathcal{A} \rightarrow E}<\infty
$$

for every $X$ in the unit ball of $E(\mathcal{M}, \tau)^{\times}$(see Sect. 2.2 and [56, Theorem 2]).

Fix $X \in E(\mathcal{M}, \tau)^{\times}$with $\|X\|_{E^{\times}} \leq 1$. Let $Z$ be an arbitrary operator in $L_{1}(\mathcal{M}, \tau) \cap$ $\mathcal{M}$ such that $Z \prec \prec X$. Since $E(\mathcal{M}, \tau)^{\times}$is a fully symmetric space (see e.g. [28, Theorem 27] or [27, Proposition 3.7]), it follows that $Z \in E(\mathcal{M}, \tau)^{\times}$and $\|Z\|_{E^{\times}} \leq 1$. Hence, $\left\|T_{i} Z\right\|_{1}=\tau\left(\left|T_{i} Z\right|\right) \stackrel{(8.1)}{\leq}\|\delta\|_{\mathcal{A} \rightarrow E}<\infty$. Since $\left\|T_{i}\right\|_{\infty} \leq\|\delta\|_{\mathcal{A} \rightarrow \mathcal{M}}<\infty$ (see [56, Theorem 2]) and $Z \in L_{1}(\mathcal{M}, \tau)$, it follows from [3, Lemma 2.5] that $T Z \in L_{1}(\mathcal{M}, \tau)$ with $\|T Z\|_{1} \leq\|\delta\|_{\mathcal{A} \rightarrow E}<\infty$. Noting that $X, T \in L_{1}(\mathcal{M}, \tau)+\mathcal{M}$ (see e.g. [28, Lemma 25]), it follows from [25, Theorems 3.10 and 4.12] that

$$
\tau(|T X|) \leq \sup \left\{\tau(|T Z|): Z \in L_{1}(\mathcal{M}, \tau) \cap \mathcal{M}, Z \prec \prec X\right\} \leq\|\delta\|_{\mathcal{A} \rightarrow E} .
$$

Since $X \in E^{\times}(\mathcal{M}, \tau),\|X\|_{E^{\times}} \leq 1$, is arbitrary and $E(\mathcal{M}, \tau)=\left(E(\mathcal{M}, \tau)^{\times}\right)^{\times}$, it follows that $T \in E(\mathcal{M}, \tau)$, as required.

Now, consider the general case. Let $c_{E}$ be the carrier projection of $E(\mathcal{M}, \tau)$. Then, $\mathcal{M}_{c_{E}}$ is a von Neumann algebra with identity $c_{E}$. By Corollary 8.2 , there is a $T \in$ $\mathcal{C}_{0}(\mathcal{M}, \tau)$ such that $\delta=\delta_{T}$ on $\mathcal{A}$. Note that $c_{E}$ is a central projection in $\mathcal{M}$ (see [28, Corollary 6]). Hence, $E\left(\mathcal{M}_{c_{E}}, \tau\right):=E(\mathcal{M}, \tau) \subset S\left(\mathcal{M}_{c_{E}}, \tau\right)$ is a strongly symmetric space having the Fatou property and $\delta_{T}: \mathcal{A}_{c_{E}} \rightarrow E\left(\mathcal{M}_{c_{E}}, \tau\right) \cap \mathcal{M}_{c_{E}}$ is also a derivation. By the first part of the proof, there is a $K \in E\left(\mathcal{M}_{c_{E}}, \tau\right) \cap \mathcal{M}_{c_{E}}$ such that $\delta_{T}=\delta_{K}$ on $\mathcal{A}_{c_{E}}$. For every $X \in \mathcal{A}$, we have $\delta_{T}(X) \in E(\mathcal{M}, \tau)$ and therefore $c_{E} \delta_{T}(X)=\delta_{T}(X)$ (see [28, Corollary 6]). Hence, for every $X \in \mathcal{A}$, we have $\delta_{T}\left(c_{E}^{\perp} X\right)=c_{E}^{\perp} \delta_{T}(X)=$ $c_{E}^{\perp} c_{E} \delta_{T}(X)=0$ and therefore,

$$
\delta(X)=\delta_{T}(X)=\delta_{T}\left(\left(c_{E}+c_{E}^{\perp}\right) X\right)=\delta_{T}\left(c_{E} X\right)+\delta_{T}\left(c_{E}^{\perp} X\right)=\delta_{K}\left(c_{E} X\right) .
$$

Since $c_{E}$ is a central projection in $\mathcal{M}$ and $c_{E} K=K=K c_{E}$, it follows that $\delta(X)=$ $\delta_{K}\left(c_{E} X\right)=\delta_{K}(X)$. Noting that $K \in E\left(\mathcal{M}_{c_{E}}, \tau\right) \cap \mathcal{M}_{c_{E}}=E(\mathcal{M}, \tau) \cap \mathcal{M}$, we complete the proof.

Since the non-commutative $L_{p}$-spaces $(1 \leq p \leq \infty)$ are strongly symmetric spaces with the Fatou property (see e.g. [28, Section 3.4]), the above theorem is a unification and extension of the results due to Johnson and Parrott [37], due to Kaftal and Weiss [44] and due to Popa [54]. Actually, the majority of Banach symmetric spaces used in analysis are strongly symmetric, and most of them have the Fatou property. Theorem 8.3 give an affirmative answer to Question 1.1 for 'almost' every proper symmetric ideal $\mathcal{E}$ in $\mathcal{M}$, that is, for most proper symmetric ideals $\mathcal{E}$ in $\mathcal{M}$, derivations from an arbitrary von Neumann subalgebra of $\mathcal{M}$ into $\mathcal{E}$ are automatically inner. One should note that the class of symmetric ideals characterized in this paper covers almost every ideal $\mathcal{E}$ corresponding to a symmetric function space in the sense of Calkin (see $[45,47])$. 
In the meantime, the ideal $\mathcal{J}(\mathcal{M})$ of all compact operators in $\mathcal{M}$ is not corresponding to any symmetric function space whenever $\mathcal{M} \neq \mathcal{J}(\mathcal{M}) \neq \mathcal{C}_{0}(\mathcal{M}, \tau)$ (see e.g. [55, Section 8]).

Acknowledgements. The authors would like to thank Erik Christensen for useful comments on the existing literature and helpful discussions, Ken Dykema, Marius Junge and Dmitriy Zanin for helpful discussions, and Sorin Popa and Florin Rădulescu for explanations of the proof in [55] and [32]. The second author was supported by Australian government scholarship. The third author and the fourth author were supported by the Australian Research Council (FL170100052).

Funding Open Access funding enabled and organized by CAUL and its Member Institutions.

Open Access This article is licensed under a Creative Commons Attribution 4.0 International License, which permits use, sharing, adaptation, distribution and reproduction in any medium or format, as long as you give appropriate credit to the original author(s) and the source, provide a link to the Creative Commons licence, and indicate if changes were made. The images or other third party material in this article are included in the article's Creative Commons licence, unless indicated otherwise in a credit line to the material. If material is not included in the article's Creative Commons licence and your intended use is not permitted by statutory regulation or exceeds the permitted use, you will need to obtain permission directly from the copyright holder. To view a copy of this licence, visit http://creativecommons.org/licenses/by/4.0/.

Publisher's Note Springer Nature remains neutral with regard to jurisdictional claims in published maps and institutional affiliations.

\section{Appendix A. Some Remarks On the Proof of [55]}

When considering derivations into the ideal $\mathcal{C}_{0}(\mathcal{M}, \tau)$ of all $\tau$-compact operators in $\mathcal{M}$, it was natural that the remarkable paper [55] on derivations into the ideal $\mathcal{J}(\mathcal{M})$ of all compact operators in $\mathcal{M}$ came into our mind. At first, we thought that following their proof step by step would be a right way to prove the derivation problem for $\mathcal{C}_{0}(\mathcal{M}, \tau)$ (actually, our proof is different from theirs and we only need the main result in [55] and some techniques used in [55, Section 4]). However, comprehending the delicate proof was a tough job to us. We would like to express our gratitude to those who have helped us in understanding the proof. They are Professors Erik Christensen, Ken Dykema, Marius Junge, Sorin Popa and Florin Rădulescu.

One of the difficulties we encountered is a technical inequality in [55, Section 7.4] (see 10th line from the bottom of Page 510), which took us several months to understand (even though, in our final version, we do not need this technique). Thanks to the hint given by Professor Florin Rădulescu, we are able to "fill the gap" in [55, Section 7.4] in our understanding, using our recent paper [10] and involving an additional reduction step. Our proof is of interest in its own right. We provide our "understanding" below for the sake of the reader.

From now on, we adopt the notations used in [55, Section 7.4]. Let $\mathcal{M}$ be a $\sigma$ finite semifinite von Neumann algebra with a semifinite faithful normal trace $\varphi$ (and a normal faithful state $\psi$ on center $\mathcal{Z}(\mathcal{M})$ of $\mathcal{M}$ ) and let $\mathcal{N}$ be a separable $I I_{1}$ von Neumann subalgebra of $\mathcal{M}$, and $\mathcal{N}^{\prime} \cap \mathcal{M}$ contains no finite projections of $\mathcal{M}$. Moreover, $\delta: \mathcal{N} \longrightarrow \mathcal{J}(\mathcal{M})$ (the ideal generated by all finite projections in $\mathcal{M}$ ) is a skew-adjoint derivation such that $\delta(\mathcal{Z}(\mathcal{N}))=\{0\}$ (see [55, The 2nd paragraph of Section 7.4]). Hence, for every $p \in \mathcal{Z}(\mathcal{N})$, we have $\delta(x p)=p \delta(x) p \in p \mathcal{J}(\mathcal{M}) p$ for every $x \in \mathcal{N}$.

Recall that $K_{\delta}:=\overline{c o}^{w o}\left\{\delta(v) v^{*}: v \in U(\mathcal{N})\right\}, \mathcal{M}_{\varphi}:=\left\{x \in \mathcal{M} \mid \varphi\left(x^{*} x\right)<\infty\right\}$ and $\mathcal{M}_{\varphi, \psi}^{1}=\left\{x \in \mathcal{M}_{\varphi} \mid\|x\|_{\infty} \leq 1, \varphi\left(x^{*} x p\right) \leq \psi(p)\right.$ for all $\left.p \in \mathcal{Z}(\mathcal{M})\right\}$. For a $T \in \mathcal{M}$, $\delta_{T}$ is defined by $[T, \cdot]$. 
If one can prove that $\delta=0$, then every derivation from $\mathcal{N}$ into $\mathcal{J}(\mathcal{M})$ is inner. Assume by contradiction that $\delta$ is not inner (therefore, $\delta \neq 0$ ). In [55], it is asserted show that there is a projection $p \in \mathcal{Z}(\mathcal{N})$ and a projection $e \in \mathcal{M}_{p}$ such that $\varphi\left(e(v p)^{*} \delta(v p) e\right) \neq 0$ for some unitary element $v \in \mathcal{N}, \mathcal{N}_{p} \ni y \mapsto \varphi(y e)$ is faithful on $\mathcal{N}_{p}$ and $e$ is a finite sum of elements in $\mathcal{M}_{\varphi, \psi}^{1}$ (see 10th line from the bottom of [55, Page 510]). However, the authors of [55] did not provide detailed proof for the above assertion. With the help of Professor Florin Rădulescu, we complete the proof for the assertion, which is displayed below.

Before proceeding to the proof, we need the following proposition.

Proposition A.1. There exists a projection $p \in \mathcal{Z}(\mathcal{N})$ such that the derivation $\delta_{(p)}$ : $\mathcal{N} p \longrightarrow p \mathcal{J}(\mathcal{M}) p$ (defined by $\delta($ pxp) for every $x \in \mathcal{N})$ is inner and there is no projection $q \leq \mathbf{1}-p$ such that $\delta_{(q)}: \mathcal{N} q \longrightarrow q \mathcal{J}(\mathcal{M}) q$ is inner.

Proof. If there are no projections $p \in \mathcal{Z}(\mathcal{N})$ such that the derivation $\delta_{(p)}: \mathcal{N} p \longrightarrow$ $p \mathcal{J}(\mathcal{M}) p$ is inner, then we set $p=0$.

In the following, we assume that there exists such a non-zero projection.

Note that the subalgebra $\mathcal{N}$ is separable. The Zorn lemma implies that there exists a maximal family of non-zero pairwise disjoint projections $\left\{q_{i}\right\}_{i=1}^{N} \subset \mathcal{Z}(\mathcal{N})$, where $N=\infty$, or $N$ is a positive integer, such that

$$
\delta_{\left(q_{i}\right)}(x)=\left[d_{i}, x\right], x \in \mathcal{N}_{q_{i}}
$$

for some $d_{i} \in q_{i} \mathcal{J}(\mathcal{M}) q_{i}$ (note that we can say $x \in \mathcal{N}$ here). Moreover, [55, Lemma 4.6] implies that there exists $d_{i}^{\prime} \in q_{i} K_{\delta}$ such that $\delta_{d_{i}}=\delta_{d_{i}^{\prime}}$. Then, $\left\|d_{i}^{\prime}\right\| \leq\|\delta\|$. For the sake of convenience, we still denote $d_{i}^{\prime}$ by $d_{i}$. Let

$$
p_{i}:=\vee_{j \leq i} q_{j} \text {, and } p=w o-\lim _{i} p_{i} .
$$

Note that $D_{i}:=\sum_{j \leq i} d_{j} \in K_{\delta}$ (see Remark 3.7) and $p_{i} \delta=\delta_{D_{i}}$. Since the supports of $d_{i}$ are pairwise disjoint and every $D_{i}$ is bounded with $\left\|D_{i}\right\|_{\infty} \leq\|\delta\|_{\infty}$, it follows that $D_{i} \rightarrow{ }_{w o} D$ for some $D \in \mathcal{M}$. Moreover, since every $D_{i}$ is in $K_{\delta}$, it follows from the definition of $K_{\delta}$ that $D \in K_{\delta}$. Note that $p D_{i}=D_{i} p=D_{i}$. That is, $D_{i}=p D_{i} \rightarrow_{\text {wo }} p D$. Hence, for every $x \in \mathcal{N}$, we have

$$
p \delta(x)=w o-\lim _{i} p_{i} \delta(x)=w o-\lim _{i} \delta_{D_{i}}(x)=\delta_{p D}(x) .
$$

Moreover, by [55, Proposition 4.4], we have $p D \in p \mathcal{J}(\mathcal{M}) p$. That is, $\delta_{(p)}$ is inner.

The maximality of the family $\left\{q_{i}\right\}_{i \in I}$ implies that there is no projection $q \leq \mathbf{1}-p$ such that $\delta_{(q)}: \mathcal{N} q \longrightarrow q \mathcal{J}(\mathcal{M}) q$ is inner.

Now, back to [55, Section 7.4]. Let $p$ be the projection constructed in Proposition A.1. Since we have assumed that $\delta$ is not inner, it follows that we may suppose now that $\mathbf{1}-p>0$ and consider the reduced algebra $(1-p) \mathcal{M}(\mathbf{1}-p)$.

All conditions of [55, Section 7.4] are preserved in this reduced algebra. Indeed, let nonzero projection $\mathcal{M} \ni q \leq \mathbf{1}-p$ and $q \in(\mathcal{N}(\mathbf{1}-p))^{\prime}$. Then, $q \in \mathcal{N}^{\prime} \cap \mathcal{M}$ and therefore is an infinite projection (since $\mathcal{N}^{\prime} \cap \mathcal{M}$ contains no finite projections of $\mathcal{M}$ ). In addition, the derivation $\delta$ vanishes on a maximal abelian subalgebra $\mathcal{A}(\mathbf{1}-p) \subset$ $\mathcal{N}(1-p)$.

Take a $\varphi$-finite projection $e \in(\mathbf{1}-p) \mathcal{M}_{\varphi, \psi}^{1}(\mathbf{1}-p)$. Now, we can construct a projection $p_{n} \in(\mathbf{1}-p) \mathcal{Z}(\mathcal{N})(\mathbf{1}-p)$ and a projection $e_{n} \in p_{n} \mathcal{M} p_{n}$ (which is a finite 
sum of elements in $\left.\mathcal{M}_{\varphi, \psi}^{1}\right)$ such that the form $x \mapsto \varphi\left(x e_{n}\right)$ is faithful on $\mathcal{N} p_{n}$. Indeed, let $q \in \mathcal{N}_{\mathbf{1}-p}$ be the support of the normal form $\mathcal{N}_{\mathbf{1}-p} \ni y \mapsto \varphi(y e)$ as in [55, Section 7.4]. By Proposition A.2 below, there is a central projection $p_{n} \in \mathcal{Z}\left(\mathcal{N}_{\mathbf{1}-p}\right)$ and a finite number of unitary elements $u_{1}, \cdots, u_{k(n)}$ in $\mathcal{N}_{\mathbf{1}-p}$ such that

$$
\vee_{i} u_{i} p_{n} q u_{i}^{*}=p_{n} .
$$

Let $e_{n}$ be the support projection of $\sum_{i} u_{i} p_{n} e p_{n} u_{i}^{*}$. Since $e \in(1-p) \mathcal{M}_{\varphi, \psi}^{1}(1-p)$, it follows that $e_{n}$ is a finite sum of elements in $\mathcal{M}_{\varphi, \psi}^{1}$ (see e.g. [55, 2.2.2]). Now, we prove the faithfulness of $\mathcal{N} p_{n} \ni y \mapsto \varphi\left(y e_{n}\right)$. Let $0 \leq y \in \mathcal{N} p_{n}$ and $\varphi\left(y e_{n}\right)=0$. Therefore,

$$
\begin{aligned}
\varphi\left(y \sum_{i} u_{i} p_{n} e p_{n} u_{i}^{*}\right) & =\varphi\left(y^{1 / 2}\left(\sum_{i} u_{i} p_{n} e p_{n} u_{i}^{*}\right) y^{1 / 2}\right) \\
& \leq \varphi\left(y^{1 / 2}\left\|\sum_{i} u_{i} p_{n} e p_{n} u_{i}^{*}\right\|_{\infty} e_{n} y^{1 / 2}\right)=0 .
\end{aligned}
$$

Since $\varphi\left(y u_{i} p_{n} e p_{n} u_{i}^{*}\right) \geq 0$ for all $i$, we have that $\varphi\left(y u_{i} p_{n} e p_{n} u_{i}^{*}\right)=0$ for all $i$. Therefore, we obtain that $y u_{i} e=y u_{i} p_{n} e=0$, which implies that $u_{i}^{*} y u_{i} e=0$. Hence, we have $s\left(u_{i}^{*} y u_{i}\right) e=0$ and therefore, $\varphi\left(s\left(u_{i}^{*} y u_{i}\right) e\right)=0$. Then, $s\left(u_{i}^{*} y u_{i}\right) \leq \mathbf{1}-q$ and $u_{i}^{*} y u_{i} q=$ 0 . Hence, we have $y u_{i} q p_{n} u_{i}^{*}=0$, which implies that $y=y p_{n}=y \bigvee_{i} u_{i} q p_{n} u_{i}^{*}=0$.

Since $\delta$ is a skew-adjoint derivation, it follows that $p_{n} v^{*} \delta(v) p_{n}$ is self-adjoint for every $v \in p_{n} \mathcal{U}(\mathcal{N}) p_{n}$.

There are two possible cases:

Case 1. There exists $v \in \mathcal{U}(\mathcal{N})$ such that the support $s\left(p_{n} v^{*} \delta(v) p_{n}\right)$ of $p_{n} v^{*} \delta(v) p_{n}$ is a $\varphi$-infinite projection.

Case 2. For every $v \in \mathcal{U}(\mathcal{N}), s\left(p_{n} v^{*} \delta(v) p_{n}\right)$ is a $\varphi$-finite projection.

If $\varphi\left(e_{n} p_{n} v^{*} \delta(v) p_{n}\right) \neq 0$ for some unitary element $v$ in $\mathcal{N}$, then this $v$ is exactly the unitary element that we need. That is, $p_{n}, v$ and $e_{n}$ satisfy the assumptions in [55, Section 7.4], which implies that $\delta_{\left(p_{n}\right)}$ is inner. This contradicts the choice of $p$. Hence, we may always assume that $\varphi\left(e_{n} p_{n} v^{*} \delta(v) p_{n}\right)=0$ for all $v \in \mathcal{U}(\mathcal{N})$.

Case 1.

Suppose that $v \in \mathcal{U}(\mathcal{N})$ is such that $s\left(p_{n} v^{*} \delta(v) p_{n}\right)$ is a $\varphi$-infinite projection. Set

$$
x:=p_{n} v^{*} \delta(v) p_{n} .
$$

That is, $\varphi\left(e_{n} x\right)=0$. Since $\delta$ is skew-adjoint, it follows that $x^{*}=x$.

(a) Suppose that $f:=\left(p_{n}-e_{n}\right) \wedge s\left(x_{+}\right) \neq 0$. Then, we have

$$
\varphi(f x)=\varphi\left(f x_{+}\right)>0 .
$$

By $[55,2.2 .4]$, there exists a $\varphi$-finite projection $g \in \mathcal{M}_{\varphi, \psi}^{1}$ with $g \leq f$ such that $\varphi(g x)=\varphi\left(g x_{+}\right) \neq 0$. In particular, $g \leq f \leq s\left(x_{+}\right)$. We have that

$$
\varphi\left(\left(e_{n}+g\right) x\right)=\varphi\left(e_{n} x\right)+\varphi(g x)=0+\varphi(g x) \neq 0 .
$$

Since $\varphi\left(\cdot e_{n}\right)$ is faithful on $\mathcal{N}_{p_{n}}$, it follows that the form $y \mapsto \varphi\left(y\left(e_{n}+g\right)\right)$ is faithful on $\mathcal{N}_{p_{n}}$. Hence, $v, e_{n}+g$ and $p_{n}$ are what we need.

Similarly, we can consider the case when $\left(p_{n}-e_{n}\right) \wedge s\left(x_{-}\right) \neq 0$. 
(b). The remaining case is $\left(p_{n}-e_{n}\right) \wedge s\left(x_{+}\right)=0=\left(p_{n}-e_{n}\right) \wedge s\left(x_{-}\right)$. In this case, since

$$
e_{n} \geq\left(p_{n}-e_{n}\right) \vee s\left(x_{+}\right)-\left(p_{n}-e_{n}\right) \sim s\left(x_{+}\right)-\left(p_{n}-e_{n}\right) \wedge s\left(x_{+}\right)=s\left(x_{+}\right),
$$

it follows that $s\left(x_{+}\right) \preceq e_{n}$ (and similarly, $\left.s\left(x_{-}\right) \preceq e_{n}\right)$. Since $e_{n}$ is $\varphi$-finite, it follows that $s\left(x_{+}\right)$and $s\left(x_{-}\right)$are $\varphi$-finite projections. Therefore, $s(x)=s\left(x_{+}\right)+s\left(x_{-}\right)$is a $\varphi$-finite projection, which contradicts the choice of $v$.

\section{Case 2.}

This is the case, where the result in our recent paper [10] plays a crucial role. that

Assume that for any $v \in \mathcal{U}(\mathcal{N})$, the projection $s\left(p_{n} v^{*} \delta(v) p_{n}\right)$ is $\varphi$-finite. We have

$$
p_{n} v^{*} \delta(v) p_{n}=p_{n} v^{*} \delta(v) p_{n} s\left(p_{n} v^{*} \delta(v) p_{n}\right) \in p_{n} L_{2}(\mathcal{M}, \varphi) p_{n} \cap p_{n} \mathcal{M} p_{n}
$$

and

$$
p_{n} \delta(v) p_{n}=p_{n} v p_{n} \cdot p_{n} v^{*} \delta(v) p_{n} \in p_{n} L_{2}(\mathcal{M}, \varphi) p_{n} \cap p_{n} \mathcal{M} p_{n}
$$

for any $v \in \mathcal{U}(\mathcal{N})$. Therefore, the image $\delta$ lies in $p_{n} L_{2}(\mathcal{M}, \varphi) p_{n} \cap p_{n} \mathcal{M} p_{n}$.

Now, [10, Corollary 3.6] implies that there exists $d \in p_{n} L_{2}(\mathcal{M}, \varphi) p_{n} \cap p_{n} \mathcal{M} p_{n} \subset$ $p_{n} \mathcal{J}(\mathcal{M}) p_{n}$ such that $\delta=[d, \cdot]$ on $\mathcal{N}_{p_{n}}$. Since $p_{n} \leq \mathbf{1}-p$, we obtain a contradiction to the maximality of $p$.

Thus, supposing that $\mathbf{1}-p>0$, we obtain either contradictions or that there is a projection $p \in \mathcal{Z}(\mathcal{N})$ and a projection $e \in \mathcal{M}_{p}$ such that $\varphi\left(e(v p)^{*} \delta(v p) e\right) \neq 0$ for some unitary element $v \in \mathcal{N}, \mathcal{N}_{p} \ni y \mapsto \varphi(y e)$ is faithful on $\mathcal{N}_{p}$ and $e$ is a finite sum of elements in $\mathcal{M}_{\varphi, \psi}^{1}$.

For the sake of completeness, we present a proof for (A.1).

Proposition A.2. Let $\mathcal{N}$ be a finite von Neumann algebra and let $q$ be a projection in $\mathcal{N}$. There exists a sequence of central projections $p_{n}$ in $\mathcal{N}$, such that $p_{n} \uparrow c(q)(c(q)$ is the central support of $q$ ), and

$$
p_{n}=\bigvee_{i=1}^{n+1} u_{n, i}^{*} p_{n} q u_{n, i},
$$

where $u_{n, 1}, \ldots, u_{n, n+1}$ are unitaries from $\mathcal{N}$ for every $n \geq 1$.

Proof. Passing, if necessary, to the algebra $\mathcal{N}_{c(q)}$, we can assume that $c(q)=\mathbf{1}$.

The main tool in the proof is the theory of comparison of projections.

We firstly construct a sequence of pairwise disjoint central projections $\left\{z_{n}\right\}$ in $\mathcal{N}$ and pairwise disjoint projections $\left\{r_{n}\right\}$ inductively.

1. By [42, Theorem 6.2.7], there exists $z$ such that $z q \succeq z(\mathbf{1}-q)$ and $(1-z) q \preceq$ $(1-z)(1-q)$. We define $z_{1}:=z$ and define $r_{1}$ to be the projection such that $\left(\mathbf{1}-z_{1}\right)(\mathbf{1}-q) \geq r_{1} \sim\left(\mathbf{1}-z_{1}\right) q$.

2. Assume now that $z_{1}, \ldots, z_{n}$ and $r_{1}, \ldots, r_{n}$ are already defined. Consider projections $\left(1-z_{1}-\cdots-z_{n}\right) q$ and $\left(1-z_{1}-\cdots-z_{n}\right)\left(1-q-r_{1}-\cdots-r_{n}\right)$. [42, Theorem 6.2.7] applied in the reduced von Neumann algebra $\mathcal{N}_{\mathbf{1}-z_{1}-\cdots-z_{n}}$ guarantees that there exist $z_{n+1} \leq \mathbf{1}-z_{1}-\cdots-z_{n}$ and $r_{n+1} \leq\left(\mathbf{1}-z_{1}-\cdots-z_{n+1}\right)\left(\mathbf{1}-q-r_{1}-\cdots-r_{n}\right)$, such that $z_{n+1} q \succeq z_{n+1}\left(\mathbf{1}-q-r_{1}-\cdots-r_{n}\right)$ and $\left(\mathbf{1}-z_{1}-\cdots-z_{n+1}\right) q \sim r_{n+1}$. By construction $\left\{r_{n}\right\}$ are pairwise disjoint. 
We claim that $\sum_{n=1}^{\infty} z_{n}=\mathbf{1}$. Suppose that $z_{0}:=\mathbf{1}-\sum_{n=1}^{\infty} z_{n} \neq 0$. Since $c(q)=\mathbf{1}$, it follows that $q z_{0} \neq 0$. By the construction, $z_{0} r_{m} \sim z_{0} q \preceq z_{0}\left(\mathbf{1}-q-r_{1}-\cdots-r_{n}\right)$ for all $n, m \geq 1$. Let $\tau_{c}$ be the faithful centervalued trace on $\mathcal{N}$. We have that

$$
\tau_{c}\left(z_{0} q\right) \leq \tau_{c}\left(z_{0}\left(\mathbf{1}-q-r_{1}-\cdots-r_{n}\right)\right)=\tau_{c}\left(z_{0}\right)-(n+1) \tau_{c}\left(z_{0} q\right),
$$

that is, $0 \leq(n+2) \tau_{c}\left(q z_{0}\right) \leq \tau_{c}\left(z_{0}\right)$, and therefore $(n+2)\left\|\tau_{c}\left(q z_{0}\right)\right\| \leq 1$, for all $n \geq 1$. This contradiction implies that $\sum_{n=1}^{\infty} z_{n}=\mathbf{1}$.

We set $p_{n}=\sum_{i=1}^{n} z_{n}$. It is clear that $p_{n} \uparrow \mathbf{1}$.

Note that $z_{n}=z_{n} q+z_{n}\left(\mathbf{1}-q-r_{1}-\cdots-r_{n-1}\right)+z_{n} r_{1}+\cdots+z_{n} r_{n-1}$. All summands on the right side are pairwise orthogonal and majorized by $z_{n} q$. Hence, for every $n \geq 1$ and $k \leq n$ the projection $z_{k}$ can be decomposed in the sum of no more than $n+1$ pairwise disjoint summands majorized by $z_{k} q$. Therefore, $p_{n}=\sum_{i=1}^{n+1} e_{i}$, where $e_{i}$ are pairwise orthogonal projections and $e_{i} \preceq p_{n} q, i=1, \ldots, n+1$.

Since $\mathcal{N}$ is a finite von Neumann algebra, there exist unitaries $u_{n, 1}, \ldots, u_{n, n+1}$ from $\mathcal{N}$, such that $e_{i} \leq u_{n, i}^{*} p_{n} q u_{n, i}, i=1, \ldots, n+1$ (see e.g. [64, Chapter XIV, Lemma 2.1]). Hence, we obtain that

$$
p_{n}=\bigvee_{i=1}^{n+1} u_{n, i}^{*} p_{n} q u_{n, i} .
$$

\section{References}

1. Albeverio, S., Ayupov, S., Kudaibergenov, K.: Structure of derivations on various algebras of measurable operators for type I von Neumann algebras. J. Funct. Anal. 256(9), 2917-2943 (2009)

2. Alekseev, V., Kyed, D.: Measure continuous derivations on von Neumann algebras and applications to $L^{2}$-cohomology. J. Operator Theory 73, 91-111 (2015)

3. Azamov, N., Carey, A., Dodds, P., Sukochev, F.: Operator integrals, spectral shift, and spectral flow. Can. J. Math. 61, 241-263 (2009)

4. Bader, U., Gelander, T., Monod, N.: A fixed point theorem for $L^{1}$ spaces. Invent. Math. 189, 143-148 (2012)

5. Ber, A., Chilin, V., Levitina, G.: Derivations with values in quasinormable bimodules of locally measurable operators. Math. Tr. 17(1), 3-18 (2014). (in Russian)

6. Ber, A., Chilin, V., Levitina, G., Sukochev, F.: Derivations on symmetric quasi-Banach ideals of compact operators. J. Math. Anal. Appl. 397(2), 628-643 (2013)

7. Ber, A., Chilin, V., Sukochev, F.: Non-trivial derivations on commutative regular algebras. Extracta Math. 21(2), 107-147 (2006)

8. Ber, A., Chilin, V., Sukochev, F.: Continuity of derivations of algebras of locally measurable operators. Integr. Equ. Oper. Theory 75, 527-557 (2013)

9. Ber, A., Chilin, V., Sukochev, F.: Continuous derivations on algebras of locally measurable operators are inner. Proc. Lond. Math. Soc. 109(1), 65-89 (2014)

10. Ber, A., Huang, J., Levitina, G., Sukochev, F.: Derivations with values in ideals of semifinite von Neumann algebras. J. Funct. Anal. 272, 4984-4997 (2017)

11. Ber, A., Sukochev, F.: Derivations in the Banach ideals of $\tau$-compact operators, p. 12. arXiv:1204.4052v1

12. Ber, A., Sukochev, F.: Commutator estimates in $W^{*}$-factors. Trans. Am. Math. Soc. 364(10), 5571-5587 (2012)

13. Ber, A., Sukochev, F.: Commutator estimates in $W^{*}$-algebras. J. Funct. Anal. 262(2), 537-568 (2012)

14. Choi, Y.: On commutative, operator amenable subalgebras of finite von Neumann algebras. J. Reine Angew. Math. 678, 201-222 (2013)

15. Christensen, E.: Extension of derivations. J. Funct. Anal. 27, 234-247 (1978)

16. Christensen, E.: The $C^{*}$-algebra of generalized compacts associated with a von Neumann algebra, operators in indefinite metric spaces, scattering theory and other topics (Bucharest, 1985). Oper. Theory Adv. Appl. 24, 51-58 (1987) 
17. Christensen, E., Effros, E., Sinclair, A.: Completely bounded multilinear maps and $C^{*}$-algebraic cohomology. Invent. Math. 90, 279-296 (1987)

18. Christensen, E., Pop, F., Sinclair, A., Smith, R.: Hochschild cohomology of factors with property $\Gamma$. Ann. Math. 158, 635-659 (2003)

19. Connes, A., Shlyakhtenko, D.: $L^{2}$-homology for von Neumann algebras. J. Reine Angew. Math. 586, 125-168 (2005)

20. Conway, J.: A Course in Functional Analysis. Springer, New York (1985)

21. Davidson, K.: Nest Algebras. Longman Scientific \& Technical, Harlow (1988)

22. Dixmier, J.: Les algebres d'operateurs dans l'Espace Hilbertien, 2nd edn. Gauthier-Vallars, Paris (1969)

23. Dodds, P., Dodds, T., de Pagter, B.: Non-commutative Banach function spaces. Math. Z. 201, 583-597 (1989)

24. Dodds, P., Dodds, T., de Pagter, B.: Fully symmetric operator spaces. Integr. Equ. Oper. Theory 15, 942-972 (1992)

25. Dodds, P., Dodds, T., de Pagter, B.: Noncommutative Köthe duality. Trans. Am. Math. Soc. 339(2), 717-750 (1993)

26. Dodds, P., Dodds, T., Sukochev, F., Tikhonov, S.: A non-commutative Yosida-Hewitt theorem and convex sets of measurable operators closed locally in measure. Positivity 9, 457-484 (2005)

27. Dodds, P., de Pagter, B.: The non-commutative Yosida-Hewitt decomposition revisited. Trans. Am. Math. Soc. 364(12), 6425-6457 (2012)

28. Dodds, P., de Pagter, B.: Normed Köthe spaces: a non-commutative viewpoint. Indag. Math. 25, 206-249 (2014)

29. Dodds, P., de Pagter, B., Sukochev, F.: Sets of uniformly absolutely continuous norm in symmetric spaces of measurable operators. Trans. Am. Math. Soc. 368(6), 4315-4355 (2016)

30. Dodds, P., de Pagter, B., Sukochev, F.: Theory of noncommutative integration. Unpublished manuscript (to appear)

31. Fack, T., Kosaki, H.: Generalized $s$-numbers of $\tau$-measurable operators. Pacific J. Math. 123(2), 269-300 (1986)

32. Galatan, A., Popa, S.: Smooth bimodules and cohomology of $\mathrm{II}_{1}$ factors. J. Inst. Math. Jussieu 16(1), 155-187 (2017)

33. Hoover, T.: Derivations, homomorphisms, and operator ideals. Proc. Am. Math. Soc. 62(2), 293-298 (1977)

34. Holmstedt, T.: Interpolation of quasi-normed spaces. Math. Scand. 26, 177-199 (1970)

35. Huang, J., Levitina, G., Sukochev, F.: $M$-embedded symmetric spaces and the derivation problem. Math. Proc. Cambridge Philos. Soc. 169, 607-622 (2020)

36. Johnson, B.: Cohomology in Banach Algebras, Mem. Amer. Math. Soc., vol. 127. American Mathematical Society, Providence (1972)

37. Johnson, B., Parrott, S.: Operators commuting with a von Neumann algebras modulo the set of compact operators. J. Funct. Anal. 11, 39-61 (1972)

38. Kadison, R.: Derivations of operator algebras. Ann. Math. 83, 280-293 (1966)

39. Kadison, R., Ringrose, J.: Derivations and automorphisms of operator algebras. Commun. Math. Phys. 4, 32-63 (1967)

40. Kadison, R., Ringrose, J.: Derivations and automorphisms of operator algebras, II. J. Funct. Anal. 1, 204-221 (1967)

41. Kadison, R., Ringrose, J.: Fundamentals of the Theory of Operator Algebras I. Academic Press, Orlando (1983)

42. Kadison, R., Ringrose, J.: Fundamentals of the Theory of Operator Algebras II. Academic Press, Orlando (1986)

43. Kaftal, V.: On the theory of compact operators in von Neumann algebras I. Indiana Univ. Math. J. 26, 447-457 (1977)

44. Kaftal, V., Weiss, G.: Compact derivations relative to semifinite von Neumann algebras. J. Funct. Anal. 62, 202-220 (1985)

45. Kalton, N., Sukochev, F.: Symmetric norms and spaces of operators. J. Reine Angew. Math. 621, 81-121 (2008)

46. Krein, S., Petunin, Yu., Semenov, E.: Interpolation of linear operators (Translated from Russian by J. Szũcs. Translations of Mathematical Monographs, vol. 54). American Mathematical Society, Providence (1982)

47. Lord, S., Sukochev, F., Zanin, D.: Singular Traces: Theory and Applications. De gruyter Studies in Mathematical Physics, p. 46 (2012)

48. Losert, V.: The derivation problem for group algebras. Ann. Math. 168, 221-246 (2008)

49. Nelson, E.: Notes on non-commutative integration. J. Funct. Anal. 15, 103-116 (1974)

50. Oikhberg, T.: Hyperreflexivity and operator ideals. J. Funct. Anal. 246, 242-280 (2007) 
51. Olesen, K.: Connes embedding problem: Sofic groups and the QWEP conjecture. Master thesis, University of Copenhagen (2012)

52. Pedersen, G.: $C^{*}$-Algebras and Their Automorphism Groups. Adademic Press, London (1979)

53. Pisier, G.: Similarity Problems and Completely Bounded Maps. Springer, Berlin (2001)

54. Popa, S.: The commutant modulo the set of compact operators of a von Neumann algebra. J. Funct. Anal. 71, 393-408 (1987)

55. Popa, S., Rădulescu, F.: Derivations of von Neumann algebras into the compact ideal space of a semifinite algebra. Duke Math. J. 57(2), 485-518 (1988)

56. Ringrose, J.: Automatic continuity of derivations of operator algebras. J. Lond. Math. Soc. 5, 432-438 (1972)

57. Runde, V.: $\mathcal{B}\left(\ell^{p}\right)$ is never amenable. J. Am. Math. Soc. 23(4), 1175-1185 (2010)

58. Sakai, S.: Derivations of $W^{*}$-algebras. Ann. Math. 83, 273-279 (1966)

59. Sakai, S.: $C^{*}$-algebras and $W^{*}$-algebras. Reprint of the 1971 edition. Classics in Mathematics. Springer, Berlin (1998)

60. Segal, I.: A non-commutative extension of abstract integration. Ann. Math. 57, 401-457 (1953)

61. Sinclair, A., Smith, R.: Hochschild Cohomology of von Neumann Algebras. London Mathematical Society Lecture Note Series, vol. 203. Cambridge University Press, Cambridge (1995)

62. Takesaki, M.: Theory of Operator Algebras I. Springer, New York (1979)

63. Takesaki, M.: Theory of Operator Algebras II. Springer, Berlin (2003)

64. Takesaki, M.: Theory of Operator Algebras III. Springer, Berlin (2003)

65. Voiculescu, D.-V.: Miscellaneous on commutants mod normed ideals and quasicentral modulus I. arXiv:2008.06990v1 (2020)

66. Wright, S.: Weakly compact, operator-valued derivations of type I von Neumann algebras. Can. J. Math. 36, 436-457 (1984)

Communicated by H-T. Yau 